\title{
Current practices utilized by independent garden centers to extend the profitable season by returning customers, raising profit margins and implementing new practices
}

Nona Kay Hunt

West Virginia University

Follow this and additional works at: https://researchrepository.wvu.edu/etd

\section{Recommended Citation}

Hunt, Nona Kay, "Current practices utilized by independent garden centers to extend the profitable season by returning customers, raising profit margins and implementing new practices" (2008). Graduate Theses, Dissertations, and Problem Reports. 2631.

https://researchrepository.wvu.edu/etd/2631

This Thesis is protected by copyright and/or related rights. It has been brought to you by the The Research Repository @ WVU with permission from the rights-holder(s). You are free to use this Thesis in any way that is permitted by the copyright and related rights legislation that applies to your use. For other uses you must obtain permission from the rights-holder(s) directly, unless additional rights are indicated by a Creative Commons license in the record and/ or on the work itself. This Thesis has been accepted for inclusion in WVU Graduate Theses, Dissertations, and Problem Reports collection by an authorized administrator of The Research Repository @ WVU. For more information, please contact researchrepository@mail.wvu.edu. 
Current Practices Utilized by Independent Garden Centers to Extend the Profitable Season by Returning Customers, Raising Profit Margins and Implementing New Practices

Nona Kay Hunt

Thesis Submitted to the

Davis College of Agriculture, Forestry and Consumer Sciences at West Virginia University

in partial fulfillment of the requirements

for the degree of

Master of Science

in

Agricultural and Extension Education

Stacy A. Gartin, Ph.D., Chair

Harry N. Boone, Jr., Ph.D.

Todd P. West, Ph.D.

Division of Resource Management

Morgantown, West Virginia

2008

Key Words: Garden Center, Extending Seasons, Horticulture 


\section{ABSTRACT \\ Current Practices Utilized by Independent Garden Centers to Extend the Profitable Season by Returning Customers, Raising Profit Margins and Implementing New Practices}

Nona Kay Hunt

The purpose of this study was to identify the measures that independently owned retail garden centers subscribe to extend their profitable season beyond the spring and early summer. This study was descriptive survey research conducted with independent retail garden centers that were members of the Ohio Floriculture Association as of November 2006, and resided in West Virginia, Ohio, Maryland, Pennsylvania, Virginia and Kentucky. The ten most successful practices in getting customers to return identified by independent garden center retailers, were (in order of successfulness); customer friendly staff, knowledgeable and experienced staff, growing your own plants to allow for diverse product mix, larger sizes of annuals, summer annuals (e. g. wave petunias), neat and clean aisles and displays, perennials, customer designed containers (greenhouse grows until pickup date), larger sizes of perennials and summer blooming perennials. The ten most successful practices in raising profit margins identified by independent garden center retailers were (in order of successfulness); customer friendly staff, knowledgeable and experienced staff, growing your own plants to allow for diverse product mix, raise prices on all items, larger sizes of annuals, custom container design (e. g. hanging baskets, patio planters), display gardens, establish a "greeter" at entrance, summer annuals (e. g. wave petunias) and customer designed containers (greenhouse grows until pickup date). 


\section{ACKNOWLEDGEMENTS}

To the garden centers who were giving of their time and thoughts which allowed for the completion of this research project.

To the members of my graduate committee:

Dr. Stacy A. Gartin for his guidance throughout my entire college career and for believing in me and the goals I wish to attain.

Dr. Harry Boone Jr. for his impeccable skill as a researcher and statistician.

Dr. Todd P. West for his knowledge of the horticulture industry and enthusiasm about this research study.

To Alice Compton, the glue of the Ag Ed department. Thank you for your time spent doing all the busy work and always having a full candy dish.

To my family for pushing me to finish and always sharing their love and support.

To my friends for inviting me to relax AND for understanding when I had to decline.

To Chad for loving me and supporting me, letting me vent about school and work and for letting me take over your kitchen table as an office. I love you! 


\section{TABLE OF CONTENTS}

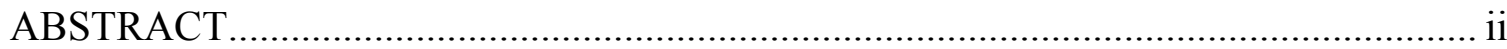

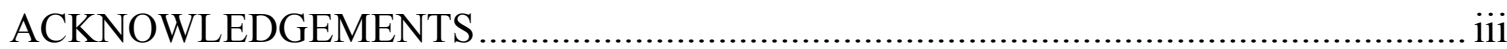

TABLE OF CONTENTS.......................................................................................... iv

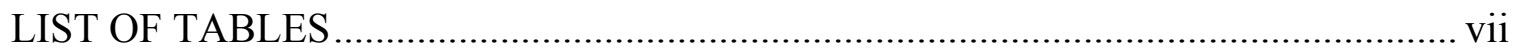

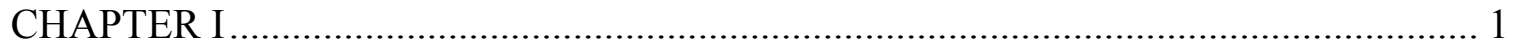

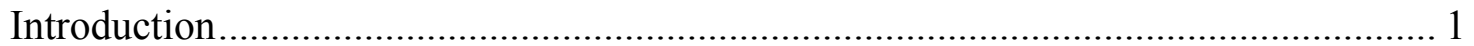

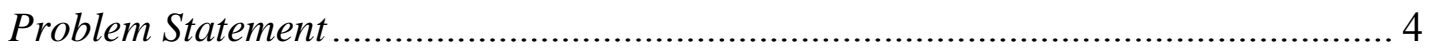

Purpose of the Study ...................................................................................... 5

Objectives of the Study .................................................................................. 5

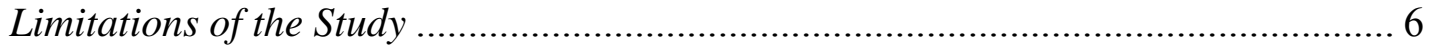

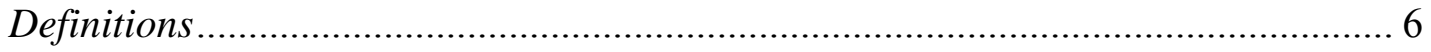

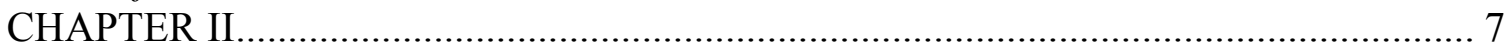

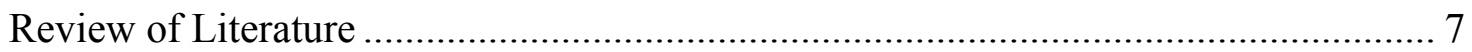

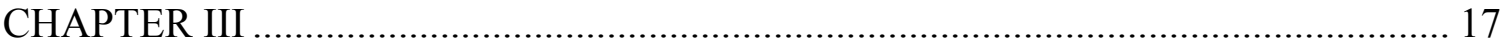

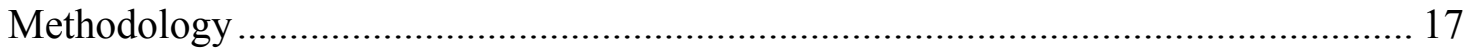

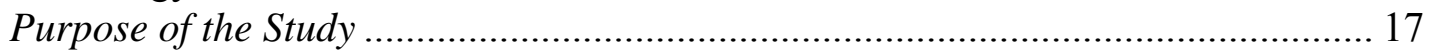

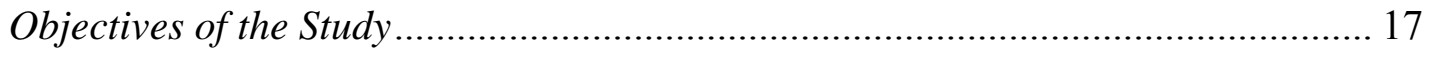

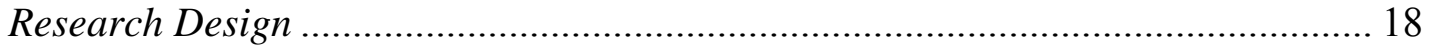

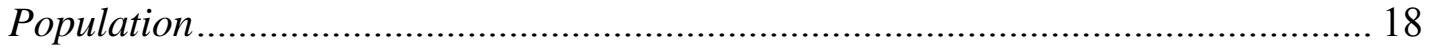

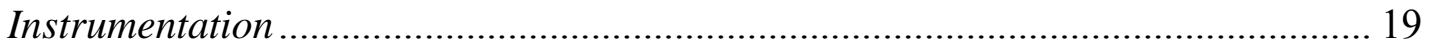

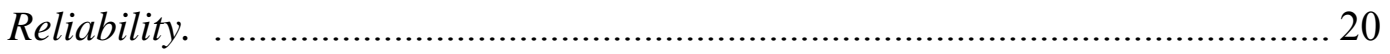

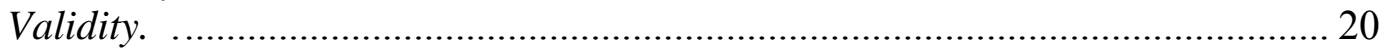

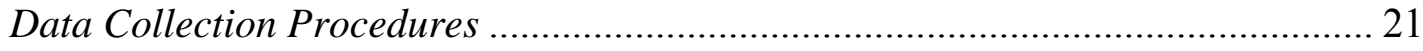

Analysis of Data ............................................................................................ 22

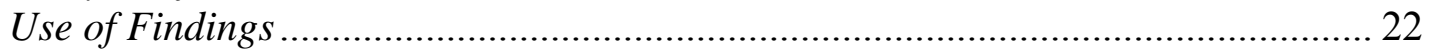

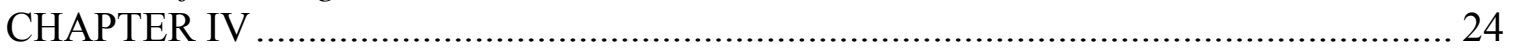

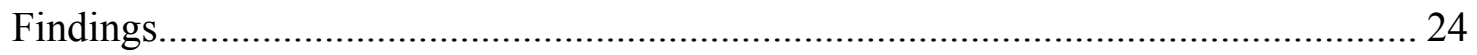

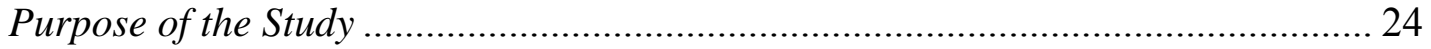

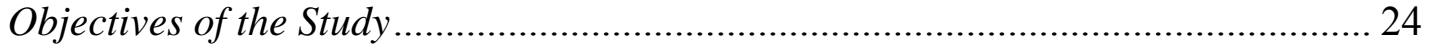

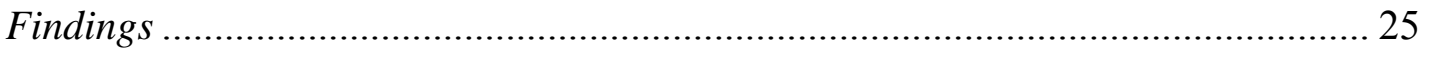

Phase I Questionnaire. .................................................................................. 25

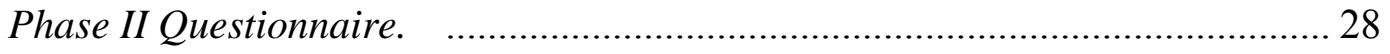

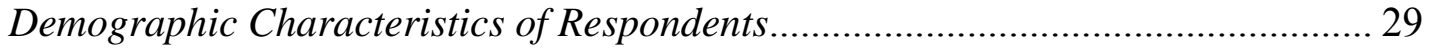

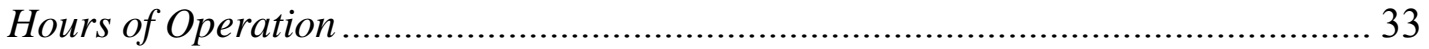

Practices Garden Centers Are Currently Using ..................................................... 37

Holiday Merchandise or Services. ................................................................. 38

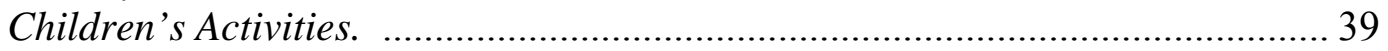

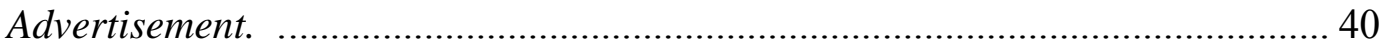

Educational Seminars. ............................................................................. 41

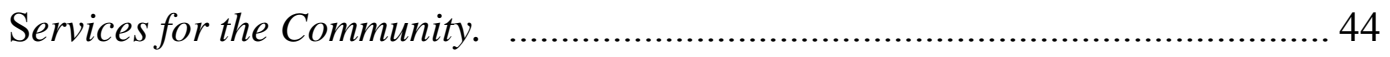

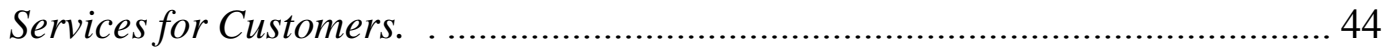

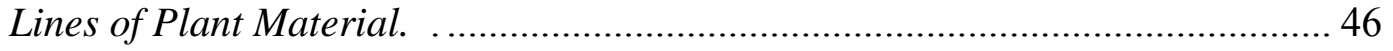

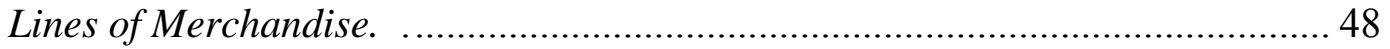

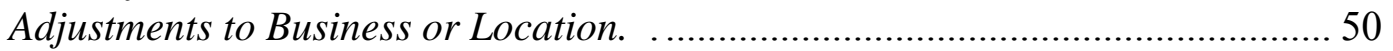


Customer Appreciation. .............................................................................. 51

Potential for Success in Getting Customers to Return to the Store ......................... 52

Holiday Merchandise or Services. .................................................................... 53

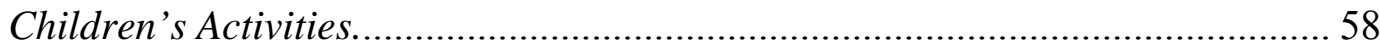

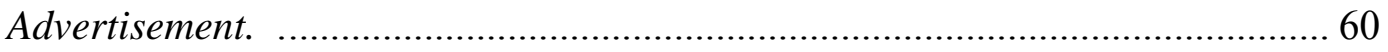

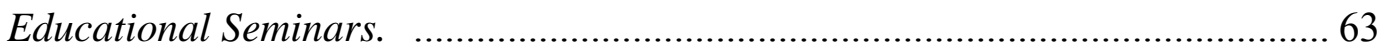

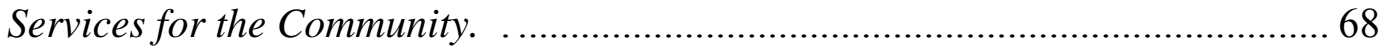

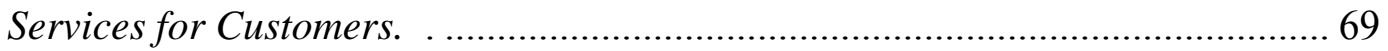

Lines of Plant Material................................................................................. 72

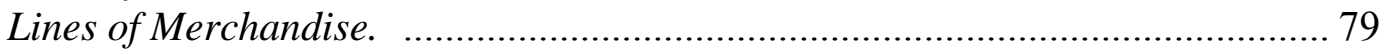

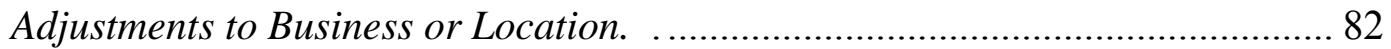

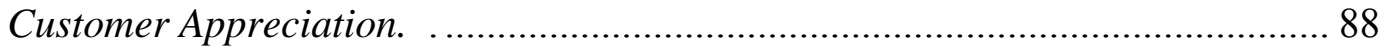

Potential for Success in Raising Profit Margins ................................................ 90

Holiday Merchandise or Services. ............................................................... 91

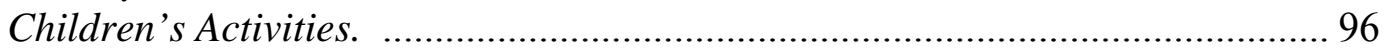

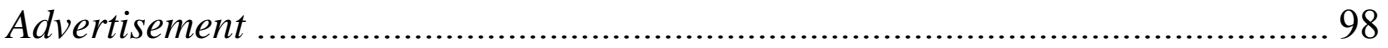

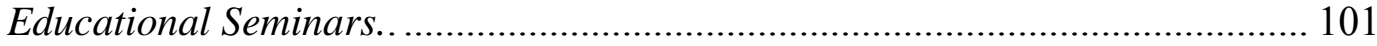

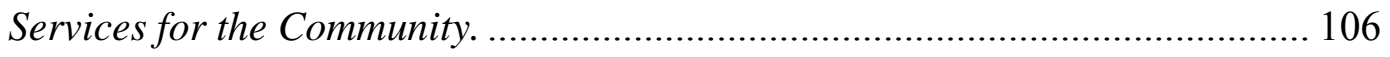

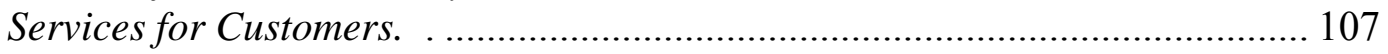

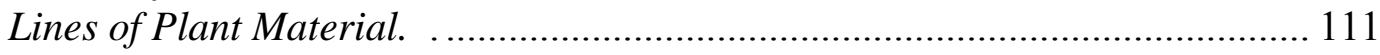

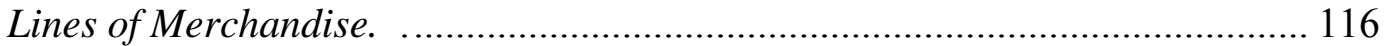

Adjustments to Business or Location. ............................................................ 120

Customer Appreciation. ............................................................................... 124

Practices Garden Centers Will Use in the Future ................................................ 126

Holiday Merchandise or Services. .............................................................. 127

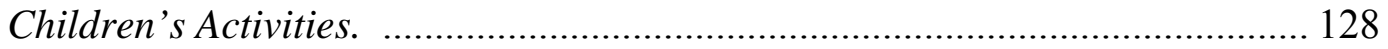

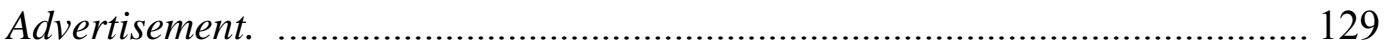

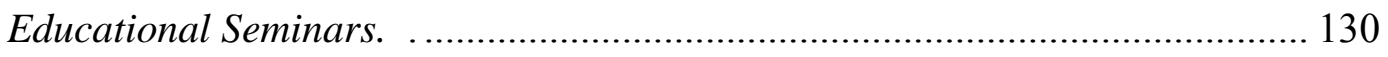

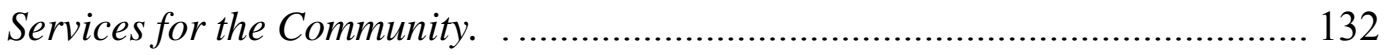

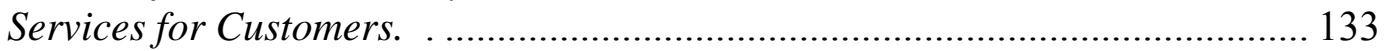

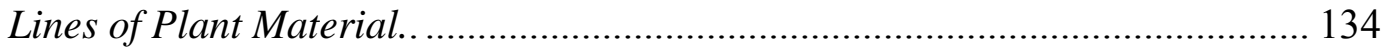

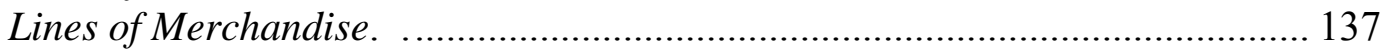

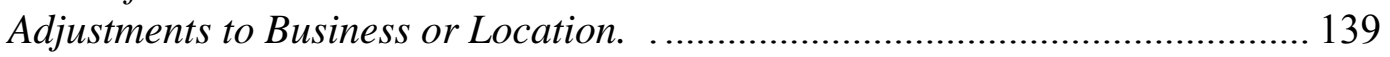

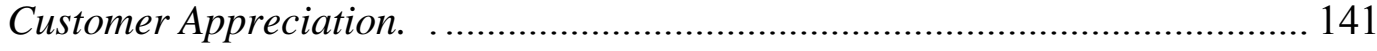

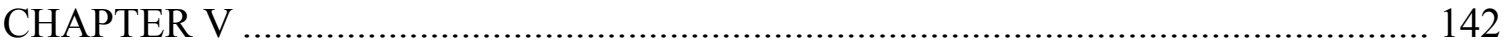

Summary, Conclusions, and Recommendations................................................. 142

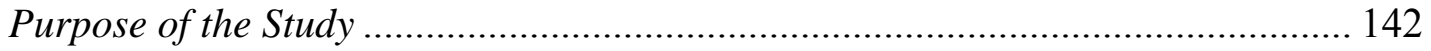

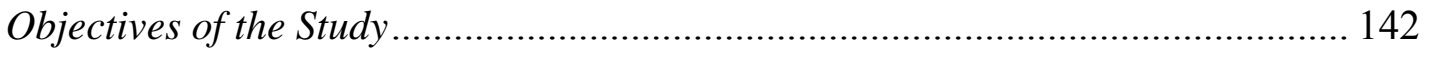

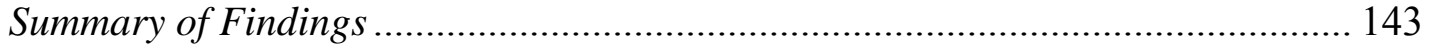

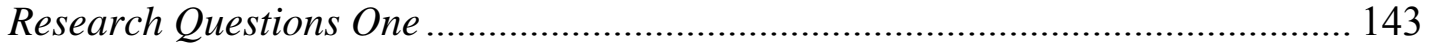

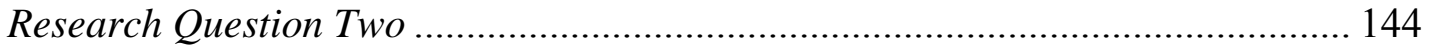

Research Question Three ............................................................................. 144

Holiday Merchandise or Services. ............................................................. 145

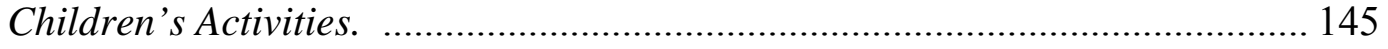

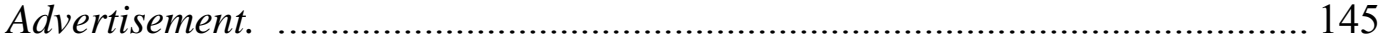

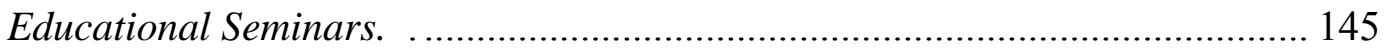


Services for the Community. ........................................................................ 146

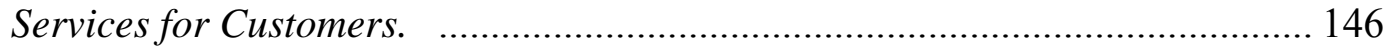

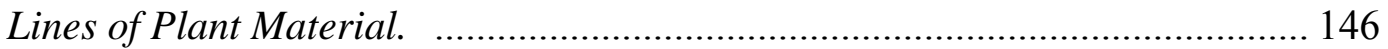

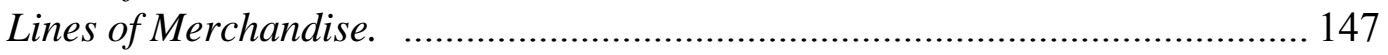

Adjustments to Business or Location. ................................................................. 147

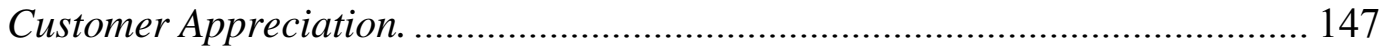

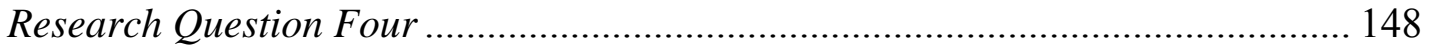

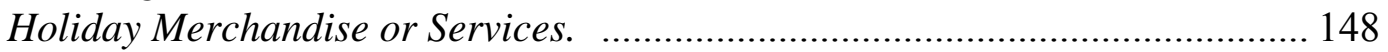

Children's Activities. ……………....................................................... 148

Advertisement. ....................................................................................... 148

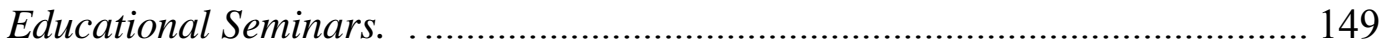

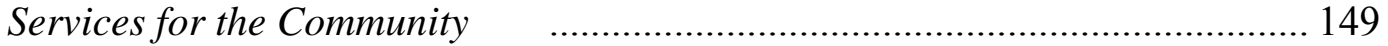

Services for Customers. . ........................................................................... 149

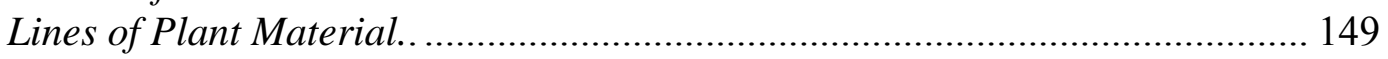

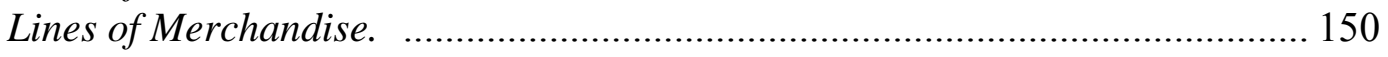

Adjustments to Business or Location. ........................................................ 150

Customer Appreciation. ......................................................................... 151

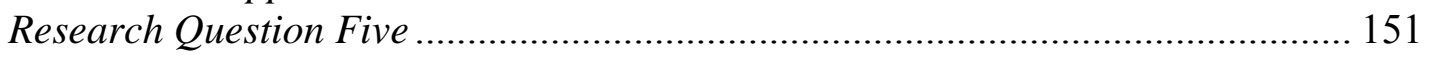

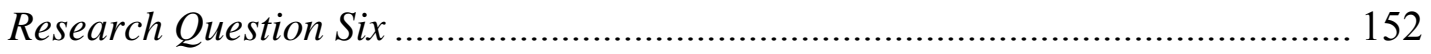

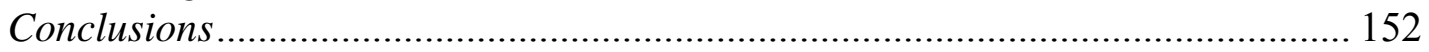

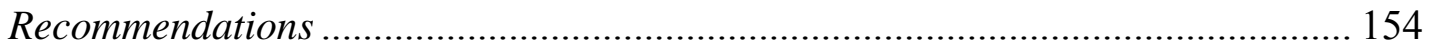

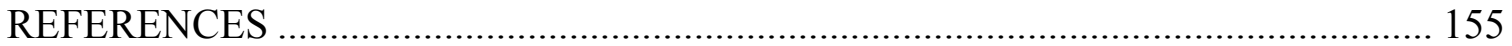

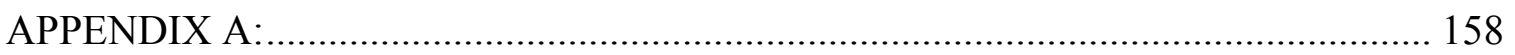

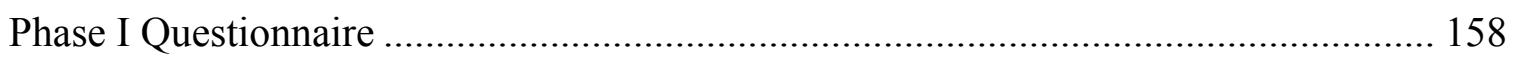

APPENDIX B:

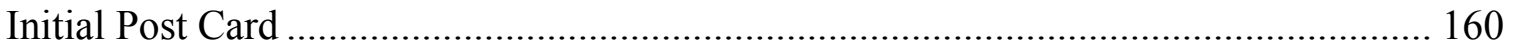

APPENDIX C:

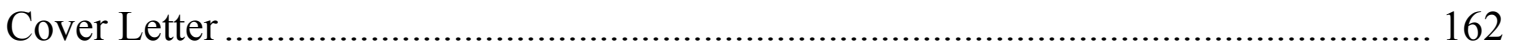

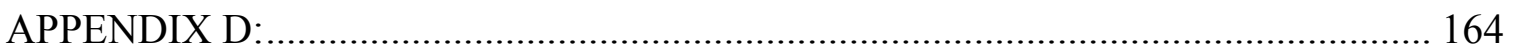

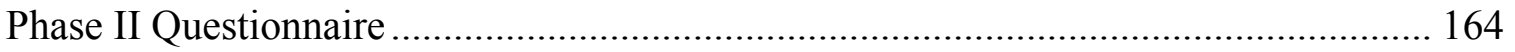

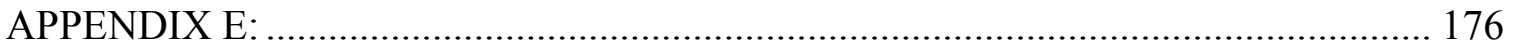

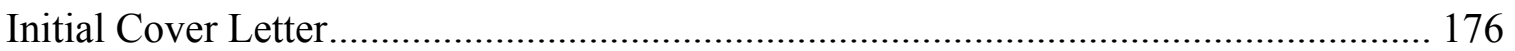

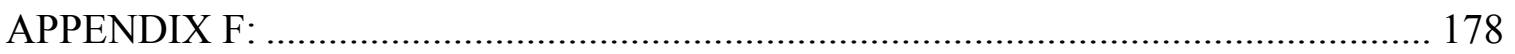

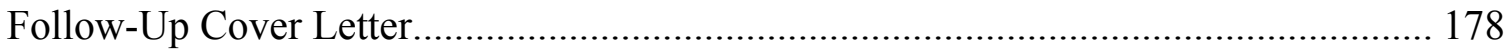

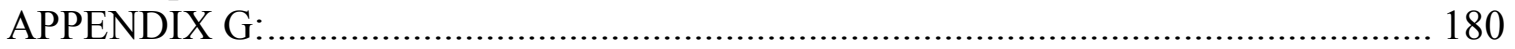

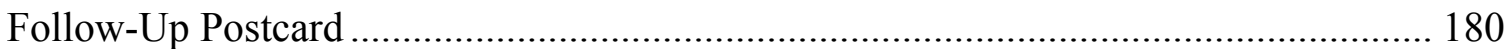

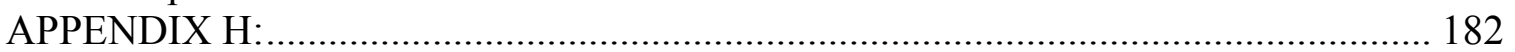

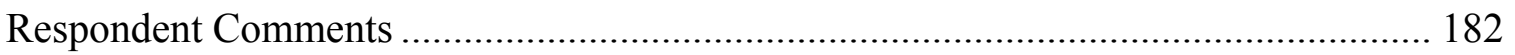

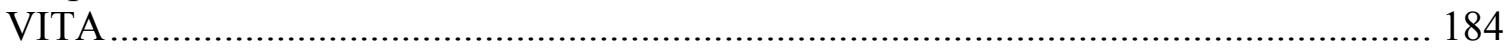




\section{LIST OF TABLES}

Table

1 Demographic Characteristics of Respondents..................................................30

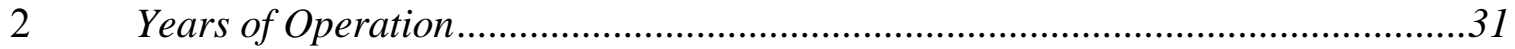

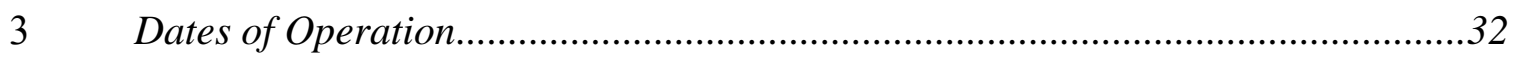

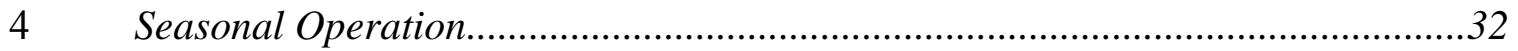

$5 \quad$ Square Footage of Garden Center..................................................................33

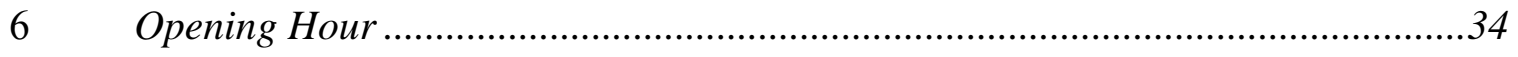

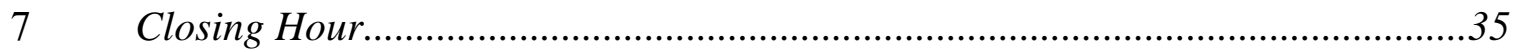

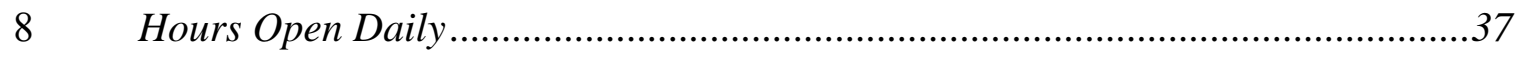

$9 \quad$ Practices Garden Centers Are Currently Using-Holiday Merchandise or

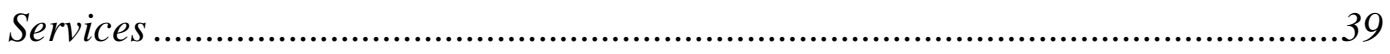

10 Practices Garden Centers Are Currently Using-Children’s Activities ................40

11 Practices Garden Centers Are Currently Using-Advertisement ........................41

12 Practices Garden Centers Are Currently Using_Educational Seminars.............43

13 Practices Garden Centers Are Currently Using—Services for the Community ....44

14 Practices Garden Centers Are Currently Using-Services for Customer.............45

15 Practices Garden Centers Are Currently Using -Lines of Plant Material ...........47

16 Practices Garden Centers Are Currently Using_Lines of Merchandise..............49

17 Practices Garden Centers Are Currently Using-Adjustments to Business or

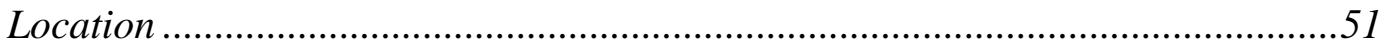

18 Practices Garden Centers Are Currently Using-Customer Appreciation...........52 
19 Potential for Success in Getting Customers to Return to the Store-Holiday Merchandise or Services.

20 Potential for Success in Getting Customers to Return to the Store-Children's Activities 60

21 Potential for Success in Getting Customers to Return to the Store-

Advertisement 62

22 Potential for Success in Getting Customers to Return to the Store-Educational

Seminars

23 Potential for Success in Getting Customers to Return to the Store-Services for the Community

24 Potential for Success in Getting Customers to Return to the Store-Services for Customers

25 Potential for Success in Getting Customers to Return to the Store-Lines of Plant Material. .78

26 Potential for Success in Getting Customers to Return to the Store-Lines of Merchandise

27 Potential for Success in Getting Customers to Return to the Store-Adjustments to Business or Location. .87

28 Potential for Success in Getting Customers to Return to the Store-Customer

Appreciation .90

29 Potential for Success in Raising Profit Margins-Holiday Merchandise or

Services .95

30 Potential for Success in Raising Profit Margins-Children’s Activities .98 
31 Potential for Success in Raising Profit Margins-Advertisement .......................100

32 Potential for Success in Raising Profit Margins-Educational Seminars ..........105

33 Potential for Success in Raising Profit Margins-Services for the Community..107

34 Potential for Success in Raising Profit Margins-Services for Customers.........110

35 Potential for Success in Raising Profit Margins_Lines of Plant Material ........115

36 Potential for Success in Raising Profit Margins_Lines of Merchandise ...........119

37 Potential for Success in Raising Profit Margins-Adjustments to Business or

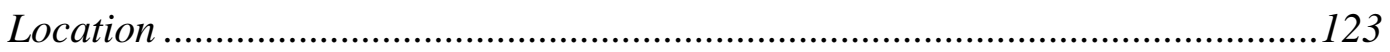

38 Potential for Success in Raising Profit Margins-Customer Appreciation ........126

39 Practices Garden Centers Will Use in the Future-Holiday Merchandise or

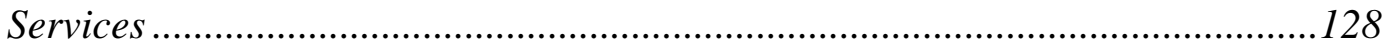

40 Practices Garden Centers Will Use in the Future-Children's Activities ..........129

41 Practices Garden Centers Will Use in the Future-Advertisement.....................130

42 Practices Garden Centers Will Use in the Future-Educational Seminars........131

43 Practices Garden Centers Will Use in the Future-Services for the

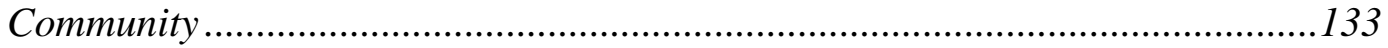

44 Practices Garden Centers Will Use in the Future-Services for Customers ......134

45 Practices Garden Centers Will Use in the Future-Lines of Plant Material ......136

46 Practices Garden Centers Will Use in the Future -Lines of Merchandise.........138

47 Practices Garden Centers Will Use in the Future-Adjustments to Business or

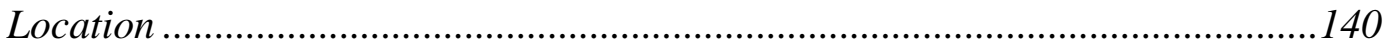

48 Practices Garden Centers Will Use in the Future-Customer Appreciation .....141 


\section{CHAPTER I}

Introduction

The bond between horticulture and humans is longstanding and all-encompassing. We encounter plants daily, at home, at work and at leisure. Our encounters include flowers in a garden, trees in the park, and fruits and vegetables on our tables. We eat the products of horticulture. We enjoy the beauty and benefits it provides and we live in an environment made better by professional horticulture.

Horticulture is a vast global industry that involves everything from nursery-plant growth to grounds maintenance, to retail production and sales. The green has a tremendous economic impact on the United States. According to Hall, Hodges and Haydu (2004), the green industry economic impacts in 2002 for the United States were estimated at $\$ 147.8$ billion in output, $1,964,339$ jobs, $\$ 95.1$ billion in value added products, $\$ 64.3$ billion in labor income, and $\$ 6.9$ billion in indirect business taxes. For the wholesale/retail sectors, total output impacts were $\$ 55.5$ billion, employment impacts were 910,104 jobs, and value added impacts were $\$ 35.5$ billion (Hall, et al., 2004). In the United States lawn and garden stores were second to landscaping services in employment at 347,916 jobs and $\$ 14.8$ billion value added products (Hall, et al., 2004).

Retail horticulture is a seasonal business in parts of the United States. The retail sector of the green industry experiences highs and lows in sales volume that is linked to the season and weather conditions. The growing season for annuals and vegetable plants in West Virginia is approximately from mid May to mid October according to the National Weather Service Forecast Office (2005). The majority of gardening done by the typical consumer is usually completed during this time because vegetables and annuals 
will have completed their growing cycles. Other states in the Northeastern part of the country experience similar growing seasons. West Virginia and its five surrounding states (Maryland, Pennsylvania, Virginia, Kentucky, Ohio) are in United States Department of Agriculture hardiness zones five, six and seven (Aker, et. al, 2005). These zones indicate the average annual minimum temperatures in these areas. The average annual minimum temperatures are as follows: zone five: $-20^{\circ}$ to $-10^{\circ} \mathrm{F}$; zone six: $-10^{\circ}$ to $0^{\circ} \mathrm{F}$; zone seven: $0^{\circ}$ to $10^{\circ} \mathrm{F}$ (Aker, et. al, 2005).

As the frenzy of planting season fades and temperatures rise, it is challenging to lure customers back to the garden center (Gilmer, 2006). It is common in many areas of the country for retailers to see vigorous spring sales, then have business drop off until it's time to sell fall mums, pansies and ornamental kales and cabbages (Cohen, 2003). When late spring is bright and blooming, the urge to plant afflicts those who love the outdoors, but the heat and fatigue factor of late July and August is undeniable (Gilmer, 2006). After the first month or two of summer when the weather is hot and dry, people don't spend as much time in the garden and plant sales tend to drop off. Cohen (2003) also noted,

Homeowners seem to have grasped the slogan, 'Fall is for Planting,' as well as realize that autumn is the time to plant bulbs for spring. But somewhere between mid-June and late August plant sales can decrease noticeably. During this down time garden centers have to be creative in getting customers to come back for more. (p. 22)

Another reason for this seasonality is that the hot and dry periods in July and August are not the best time to plant trees and shrubs. Gilmer (2006) agrees that 
"Planting during high temperatures does not result in happy, healthy transplants" (p. 19). The Better Homes and Gardens New Garden Book recommends staying away from the hot summer months when planting trees:

The best time for planting trees varies widely by location. In areas like California, you can plant trees anytime during the cool and rainy winter season. Coastal areas of California, Oregon, and Washington are bathed in moisture and trees can be planted in any season except summer. In the far North, where brutal winter conditions prevail, planting is best done in early spring as soon as the soil can be worked. In the Southeast and along the Eastern Seaboard, plant in early fall. In the Northeast, September and October are prime months for planting. In the mid-Atlantic states, October and November are best. In the Southwest, planting may be done well into December and almost anytime during winter along the Gulf Coast. In the southern Plains, plant either in early spring or autumn. Autumn and early winter are the best times to plant trees in Texas and the desert Southwest. (Aker, et. al, 2005. p.85)

Also, many retail customers are not "hard-core" gardeners and do not garden during these hot and dry periods. For these reasons as well as others, many retailers nationwide tend to lose their edge during this six- to eight-week period of the summer (Cohen, 2003). Losing edge in a retail business can be devastating. Losing edge means losing customers to competitors during any down time and may mean losing them for good. It is important for garden centers to keep the business interesting during these critical seasons so customers are attracted to return to the store repeatedly. 
Garden center retailers can do one of two things to solve this seasonality problem. The first is to cry, "Woe is me" and roll out a different sale every week (Cohen, 2003). The second is to sit down with the key players on staff and ask for thoughts on how to increase sales (Cohen, 2003). Garden centers must find ways to adapt their enterprises to extend the profitable season in order to have a higher profit margin. Gilmer (2006) states that "Every garden center's dream is to keep the spring traffic moving more evenly throughout the year so costs and cash flow do not suffer seasonal disparity" (p. 18).

Some garden centers may choose alternatives to extending the season. They may decide to shut down for an extended period of time in order to save on labor costs, utilities, etc. If garden centers do not find a way to profit by staying open in the winter or the "off-season," where will customers go for products they can't find at "big box" stores such as Wal-Mart or Lowe's? For example, many of the "big box" stores do not stock lawn and garden chemicals, house plant food or planting media year round. Not only do most independent garden centers stock these products year round, they are an excellent place for customers to shop for other gardening needs and gain important knowledge on plants and gardening as well.

\section{Problem Statement}

What measures do independent retail garden centers take to extend their profitable season beyond the spring and early summer months? What are the current practices prescribed by owners/operators of these enterprises to increase their profits? These garden centers must market themselves to consumers as year round suppliers of garden and outdoor living needs in order to succeed. In order for independently owned garden 
centers to stay in business, they must make their store the "go to" place for plants, gardening accessories and products associated with outdoor living.

Purpose of the Study

The purpose of this study was to identify the measures that independently owned retail garden centers subscribe to extend their profitable season beyond the spring and early summer. By identifying profitable practices used by garden centers in the United States, the retail horticulture industry will benefit from increased profits and more employment opportunities. The entire green industry will also benefit from the increase in retail sales. The demand for plants, planting mediums and other garden accessories will have to increase to keep up with the retail sector. The manufacturing and production of plants, mediums and accessories will create more jobs and more money in the economy.

\section{Objectives of the Study}

The objectives for the study are reflected in the following research questions:

1. What measures are currently being practiced by garden centers to extend the profitable season beyond the spring and early summer?

2. What companion products do garden centers sell to extend the profitable season?

3. Which techniques used to extend the profitable season are viewed by retailers to be most helpful in raising profit margins?

4. Which techniques used to extend the profitable season are viewed by retailers to be most helpful in returning customers to the garden center? 
5. Which techniques are retailers most likely to incorporate in their current operation?

6. Is shutting down the garden center for an extended period of time a realistic option?

\section{Limitations of the Study}

This study was limited to independent retail garden centers that were registered members of the Ohio Floriculture Association as of November 2006. Only members residing in West Virginia, Ohio, Maryland, Pennsylvania, Virginia and Kentucky were included in the study. Definitions

Garden Centers-Establishments primarily engaged in selling containerized trees, shrubs, other plants, seeds, bulbs, mulches, soil conditioners, fertilizers, pesticides, garden tools and other garden supplies to the general public and where no trees, shrubs or plants are grown on the premises (City, 2006).

Green Industry-comprised of a variety of businesses involved in production, distribution and services associated with ornamental plants, landscape and garden supplies and equipment (Hall et al., 2004).

Horticulture-the science and art of growing fruits, vegetables, flowers and ornamental plants (Mish, 1997). 


\section{CHAPTER II}

\section{Review of Literature}

Customers are the most important part of any retail business. In order for a business to be successful by any means, customers must frequent the location. Stores that have lasted through the decades have set a merchandise niche for themselves and overtime have changed customer shopping habits (Weisher, 2006). Garden centers today must shape customer shopping habits to keep shoppers returning to the store throughout the year.

"The single most consistent factor in the entire retail equation is the customer. Everything may change. Cycles may come and go. Prices and geography have less to do with customer habit than an innate and intuitive sense of what they like. Projecting an image that clearly spells out who you are and what you represent is the singular issue to customers. You must en-compass signage, lighting, color and display to create a lasting impression the first time customers see you. Their perception of your position in their lives — from necessity to frivolity — is guided by their instant visual evaluation.” (Weisher, 2006, p. 12)

Consumer trends affect the success of a retail horticulture business. Retail garden centers sell luxury. The items purchased at garden centers are not life's essentials. They are life's extras (Sharpton, 2005). Retailers must know who they are selling to and what it is that the customer wants in order to make the highest profits.

Retailers can do a variety of things to increase sales of plants throughout the year. There are many factors involved in merchandising plants (Williams, 1975). The first is to 
grow and or sale quality plants which are packaged in neat containers and attractively displayed. Quality is one of the best sales techniques. Customers will return repeatedly for quality merchandise and a successful business is supported by repeat customers.

The interior and exterior of the store should be appealing to customers as well. The aisles should be wide and the store should have a convenient, symmetrical layout (Behe et al., 1994). The merchandise should be properly displayed and clearly priced. The floor should also be kept neat and clean to promote a positive atmosphere (Behe, Pfahl, \& Hofmann, 1994). It is very important that the customer has a positive image of the store.

One way for the grower to help extend the selling season is to plan sowing and transplanting dates so that plants will be available for sales to early customers and to late customers as well as for midseason sales (Williams, 1975). Another way to increase sales is by selling companion items such as fertilizer, tools, pesticides and other gardening needs. Customers tend to buy everything they need where they get their plants because they like one-stop shopping.

Some retail garden centers experience limited success extending annual sales into the summer and expanding sales in the fall; however this does not have to be the case (Hamernik, 1997). Experts on the matter have made suggestions to improve the success rate of extending the season.

Suggestions include: landscaping with plants around the establishment to promote your product, providing large display plants to show mature specimens and ideas for combinations, providing plants for public garden displays and shows, providing seminars on a local basis and pursuing a broad customer base. (Hamernik, 1997) 
A problem that is prevalent in all retail operations is customer education. Horticulture is perhaps the most important industry where businesses should educate the consumer. Choosing the right plant and caring for it once it is installed can be much like choosing and caring for the right pet. One thing that garden centers can do to educate the consumer is to hold seminars informing the public on important topics.

The garden center should start by educating employees. It may seem bothersome to garden center employees to answer basic customer questions. "But the more information novice gardeners and landscapers have, the more likely they are to be successful with their plants—and success generates enthusiasm about planting, which, in turn, generates future purchases." (Fornari, 2005 p. 24) Employees in the garden center must be able to assist customers properly when asked for advice. It is important to hire employees with gardening or landscaping experience when possible. It is also a good idea to send employees to trade show seminars to expand their knowledge (Fornari, 2005).

A perfect time to hold seminars for customers can be the summer, winter or any slow time. Interesting and helpful seminars will help bring customers to your location. Cohen (2004) notes that "An educated customer buys throughout the entire season." (p.23) Educating customers on how to do something in the garden can be very rewarding to them as well as your bottom line.

Some popular seminars that Cohen (2004) suggests include: how to renovate an old garden or one you're not happy with; designing a new garden; learning to divide perennials; how to plant summer bulbs; pruning for beginners; pruning roses; renovating or improving your lawn; 
designing water gardens or improving existing ones; how to repot or divide aquatics; using summer flowers to make beautiful arrangements; planting containers for fall; how to dry herbs for flavorful cooking; cooking with herbs, vegetables and edible flowers; how to garden without pain; maintaining the garden in summer and fall; identifying pests and lighting design. (p. 23)

Cohen's (2004) favorite seminar topic is beginners gardening. As the seminar name suggests, these customers are going to need you to start at square one, covering topics such as kinds of tools, soil preparation and all amendments. These topics could be split into two or three seminars, giving retailers the opportunity to really demonstrate and sell products. Coupons for beginners can offer an extra incentive to buy start up items (Cohen, 2004).

Educating consumers on even the simplest topics benefit your garden center. Meghan Boyer (2007) explains the importance of not assuming your consumer will know how to apply mulch once it arrives at their homes. Garden centers should consider printing an instructional pamphlet for customers who purchase mulch. "Instructions can include application tips, problems to watch for and upkeep instructions. Make sure you warn against using too much or too little mulch" (Boyer, 2007, p. 59). Well-versed customers can lead to increased and even repeat mulch sales for the garden center.

No matter how interesting seminars may be, for a lecture series to be most successful, it must be well-publicized. Routine posting of events in the store, printed handouts available at the cash registers and a calendar of events in your newsletter augment advertising as well (Fornari, 2005). Consider making education part of an 
employee's job description. Having someone on staff responsible for writing newsletters, copying handouts, filling fact sheet dispensers and scheduling speakers will ensure education plans are coordinated and well executed (Fornari, 2005).

Although bringing customers to the garden center to educate them seems to be the most brilliant approach, giving customers something to take home can also be a great idea. Handouts available in the garden center on most frequent topics of question can be a great asset to the customer and the garden center (Fornari, 2005). If employees don't have time to spend with every customer, they can refer them to a handout on the topic.

Newsletters can also be helpful to the customer. Frequently asked questions and seasonal dilemmas can be addressed in a company newsletter (Fornari, 2005). This publication can also contain ads and promotions of special events at the garden center. Fornari (2005) also suggests setting up an information station to display handouts, newsletters and in-store reference books.

Another great way to extend the gardening season is to focus on plants that show their beauty in seasons other than spring and summer. Offering plant materials throughout the year will ensure your customers know they can garden all year long instead of just in the spring. Gilmer (2006) believes that autumn is the most counterintuitive gardening season. Customers are in back-to-school mode or settling into hibernation for the winter, however some species of plants fare much better when fall planted. Maples and burning bush virtually sell themselves in this season. Asters, ornamental grasses and cultivars from the genus Hydrangea are great late blooming flowers. "With the entire winter and wet spring to adapt to native soil and spread outside 
the nursery-container-sized rootball, the plant becomes established and drought resistant sooner in its lifespan." (Gilmer, 2006, p. 19)

To help brighten up a dreary winter, try stocking some plants that work well for interior holiday décor and living gifts. Though the ground may not be workable, the container market thrives during the winter months. "Topiaries large and small are in high demand now, particularly conical shapes and the spiral cut junipers that make hauteliving Christmas trees" (Gilmer, 2006, p. 19). Gilmer also notes one benefit of coniferous evergreens is their cold tolerance, which makes for outstanding entry accents and potted terrace displays throughout the winter and can become permanent focal points in the outdoor garden come spring. Some great varieties are blue spruce, golden arborvitaes and exotic threadleaf junipers (Gilmer, 2006).

Bonsai specimens are an aesthetic choice and make great gift items as well. Winter stock will consist mainly of coniferous evergreens which are affordable and readily available (Gilmer, 2006). Offering a "make-a-quasi-bonsai" class takes the mystery out of the uniquely pruned plants and sells a lot of small-container evergreens, rocks, decorative gravel and pricey pots during the slow season. Succulents are also a great plant to work into the greenhouse during the winter season (Gilmer, 2006). Orchids can also help diversify the product line in the garden center (Brooks, 2006). Orchids are a popular trend among house plants and come in a variety of colors suitable for every homeowner.

Garden centers can start off the spring season much earlier by adding some naturally early blooming shrubs and trees. New improved varieties of old-fashioned 
early flowering shrubs are readily available. Some popular early spring bloomers are forsythia, lilac, willows and hellebores (Gilmer, 2006).

Spring bulbs can be a powerful force for turning casual seasonal visitors into long-term customers. The reason is simple: Bulbs are easy to plant and grow successfully (Caras \& Ferguson, 2004). Even non-gardening customers can enjoy gardening success by planting a small bed of bulbs. After enjoying their colorful and inspiring display of tulips and daffodils, chances are they'll be back; ready to stock up on summer annuals, perennials, pots, potting soil and other gardening supplies (Caras \& Ferguson, 2004). Making fall bulbs work for your store means creating appealing and accessible displays and above all, having knowledgeable retail personnel on hand to answer questions. New customers will have many questions on the basics of planting and caring for bulbs.

Seasons and holidays offer garden centers the perfect opportunity to draw customers to the location. Getting the customer to the garden center once is the easy part, but getting the customer to return is the most important and most difficult part.

Fall and Halloween are prime decorating times for homeowners. It is easy for garden centers to cash in on pumpkins, fall mums, gourds and all other fall decorating items. Often times these are combined with event weekends featuring harvest themes, hayrides and refreshments. These displays can really draw a crowd and are very nice profit makers at a time when green sales are slowing down (Caras \& Ferguson, 2004). Savvy retailers know that many of these casual fall visitors aren't really gardeners-not their long term customers. Retailers also know that potential return customers or 
wannabe gardeners are also in the crowd (Caras \& Ferguson, 2004). The key is to spark an interest in gardening with these customers by inviting them to such events.

"More and more, we find retailers tying their marketing strategy to Halloween," says Tom Holliday, president of the Retail Advertising and Marketing Association. (Shoemaker, p. 15, 2003) Halloween is one of the most lucrative holidays nationwide, bringing in approximately $\$ 6.9$ billion in retail sales. The National Retail Federation (NRF) says consumers will spend roughly $\$ 44$ per household on candy, costumes and decorations (pumpkins and candles), with 18-34 year olds spending around \$67 (Shoemaker, 2003). Other ideas for turning out customers are hosting a haunted greenhouse and offering specials to customers in costume.

The color of a holiday marketing strategy or line of products can make a world of difference. Almost everyone in America when asked about the colors of fall would say oranges, reds, golds, browns, deep greens and black. People say pastel pink, lavender, lemony yellow, bright green, fluffy white and sky blue when asked about spring (Speichert, 2005). These color trends explain the difficulty retailers sometimes have selling asters and pansies and some colors of mums in the fall.

People grow accustomed to certain colors for certain times of the year. Retailers must lead by example to change the way customers think. Try planting a bed of asters and pots of pansies as part of your fall displays (Speichert, 2005). "It's just like going into any clothing store, I always buy what's on the mannequins, “ explains Matt Horn, co-owner of Matterhorn Nursery Inc., Spring Valley, NY. "People buy what we have in our display gardens... The more display gardens we put in, the more display gardens we 
sell" (Landicho, 2003 p. 20). It may take a few years for customers to accept and adapt to these color changes but it will pay off for you.

Christmas is a huge holiday for most garden centers. The typical live and artificial trees, ornaments, wreaths, swags and other decorations are easy to sell. But one business is stepping outside the box this Christmas. From October until February Dan Combs and his staff install and decorate more than 300 trees in homes and businesses in the Charleston, West Virginia area (Childress, 2006). What was once a holiday family tradition has now become a profit making adventure. Combs' company has tree decorating down to a science. It only takes about an hour to completely install and decorate a 10-foot tree (Childress, 2006). Dynamic Displays works with the customer to create a design and installation timeline that fits their business and their needs. Combs' also provides storage facilities for clients during the off-season (Childress, 2006).

Garden centers often sell poinsettias to go along with Christmas trees and other holiday decorations and gifts. A new idea in the Christmas decorations market seems to have taken a lesson or two from the florist. Painted poinsettias have been a success for the Matterhorn Nursery in Spring Valley, New York and K\&W Greenery in Janesville, Wisconsin (Calkins, 2006). According to head grower, Noah Schwartz, as many as 30 percent of the poinsettias sold in 2005 at the Matterhorn were painted. "The most popular colors were ice blue, tricolors, glittered red, snow and gold sheen" (p. 34). It is tough to predict how long this trend may last but some retailers are making it work.

If all else fails and customers are still not making routine visits to your store, try establishing a loyalty program. A loyalty program gives customers a real reason to return to the store. Kick off the loyalty program with a special event and offer a discount that 
reward customers who sign up (Shoemaker, 2003). Some people are "carrot chasers" and will do anything to get a discount or special deal.

A loyalty program needs to be easy to understand and participate in. Signs and brochures explaining the program displayed throughout the store can also encourage customers (Shoemaker, 2003). Customers must be able to comprehend the program before they will sign up and use it. At Corso's Flower \& Garden Center, the loyalty program works like this; 10 points for every dollar spent whether it's on regular or salepriced items. Every time a customer reaches 1,000 points, the get a $\$ 5$ credit (Shoemaker, 2003). These rewards could have dates of validity if a store wanted customers to return at certain times of the year.

Attracting customers to a new or older garden center usually involves stepping outside the box and getting creative. In order for garden centers to compete with other major retailers, they must be in tune with consumer trends and be willing to take chances. The garden center that is most profitable will have some set backs and ideas that don't always work. However, the most profitable garden center will keep generating new ideas and hard work and perseverance will pay off! 


\section{CHAPTER III}

\section{Methodology}

\section{Purpose of the Study}

The purpose of this study was to identify the measures that independently owned retail garden centers subscribe to extend their profitable season beyond the spring and early summer. By identifying profitable practices used by garden centers in the United States, the retail horticulture industry will benefit from increased profits and more employment opportunities. The entire green industry will also benefit from the increase in retail sales. The demand for plants, planting mediums and other garden accessories will have to increase to keep up with the retail sector. The manufacturing and production of plants, mediums and accessories will create more jobs and more money in the economy.

\section{Objectives of the Study}

The objectives for the study are reflected in the following research questions:

1. What measures are currently being practiced by garden centers to extend the profitable season beyond the spring and early summer?

2. What companion products do garden centers sell to extend the profitable season?

3. Which techniques used to extend the profitable season are viewed by retailers to be most helpful in raising profit margins?

4. Which techniques used to extend the profitable season are viewed by retailers to be most helpful in returning customers to the garden center? 
5. Which techniques are retailers most likely to incorporate in their current operation?

6. Is shutting down the garden center for an extended period of time a realistic option?

\section{Research Design}

A descriptive research design was chosen for this study. According to Ary, Jacobs, Razavieh and Sorensen (2006), "descriptive research allows for the researcher to summarize the characteristics of different groups or to measure their attitudes and opinions toward some issue" (p. 31). Descriptive research allows for a wider scope of information to be collected from a larger population, deals with real situation and allows for more specific problems to be identified. The disadvantages of using descriptive research are as follows: descriptive research has more superficial answers that are collected, descriptive research demands a lot of time and money, lacks external validity and may it may be difficult to obtain valid data (measurement error is a problem). A questionnaire was used to collect information on practices subscribed by independent retail garden centers to extend the profitable season.

\section{Population}

The target population for this study was all independent retail garden centers in the north-east region of the United States. The accessible population for this study was all independent retail garden centers that were members of the Ohio Floriculture Association (OFA) as of November 2006. OFA is a member-focused national organization of greenhouse growers, garden center operators, retail and wholesale florists, interior plantscapers, green industry suppliers, students and educators. (OFA, 
2006) OFA has members in all fifty states and is a reliable source for information. Contact information for members of OFA was readily available through contacts at the association.

Based on the Krejcie and Morgan (1970) table for determining sample size for research activities a sample population of 248 participants was randomly selected using the Statistical Package for the Social Sciences (SPSS). The random sample was reviewed to determine if all six states involved in the study were represented. Sampling error was avoided by using random selection.

Frame errors were avoided by obtaining an official list of the independent retail garden centers that were members from the Ohio Floriculture Association as of November 2006. Only one list was used for the population. Evaluation of the member list was conducted to be sure there were no duplicates that would result in a selection error. Measurement error was avoided by insuring that the instrument was reliable and valid.

\section{Instrumentation}

A modified Delphi technique was used to generate the questionnaires for this study. The first questionnaire consisted of two questions designed to gather information about the practices used by independent retail garden centers to extend the profitable season. The questions were: (a) What are the three best strategies, directly related to horticulture, that you use to extend the profitable season of your garden center? And (b) What are the three best strategies, NOT related to horticulture, that you use to extend the profitable season of your garden center? 
The statements from the first questionnaire were collected, compiled, and sorted. The statements were used to construct the questionnaire used in the second part of the study. The second questionnaire was used to measure the opinions of garden center managers related to the effectiveness of the suggested practices identified in phase one of the research. The participants were asked to rate each practice using the following scale: (4) Successful, (3) Moderately Successful, (2) Slightly Successful, or (1) Unsuccessful. The second questionnaire also collected demographic information including years of employment, annual sales, square footage of the garden center, hours of operation and to which industry magazines retailers subscribe.

Reliability. Reliability defines the trustworthiness of an instrument. Internal consistency is concerned with the idea that single items in the instrument converge to measure what the entire instrument purports to measure. The split-half method was used to determine the internal reliability of the instrument. The unequal-length SpearmanBrown coefficient was .51. According to Robinson (1991) a criterion rating of .30 or better is considered to have exemplary reliability.

Validity. Validity is an evaluative judgment of the extent to which an instrument measures what it purports to measure. The validity of the research was insured by the following steps. The instrument was revised in detail by a panel of experts. The panel of experts consisted of two agricultural and extension education professors and one horticulture professor. The experts evaluated the instrument on its content validity to see that it measured relevant content. The experts also evaluated the face validity of the instrument. The experts determined the adequacy to which the content was presented and at how the instrument was constructed. 


\section{Data Collection Procedures}

The tailored design methods of data collection by Dillman (2000) were followed in this research study. The first step in the data collection process was to send a pre-card informing the subjects they would receive the questionnaire. The second step was to make the initial mailing of the packet including a cover letter, the instrument, and stamped self addressed envelope. Each envelope was coded for follow-up purposes only. Participants were asked to identify three strategies or practices used to extend the profitable season of their retail garden center. A follow-up postcard was sent to nonrespondents after the initial return deadline. One week after the postcard, a second complete mailing was sent to non-respondents. A follow-up postcard was sent after the second deadline to non-respondents encouraging them to respond to the survey.

The panel of experts synthesized and combined the responses into 145 statements which comprised the second questionnaire along with statements from the literature review. The participants were asked to rank each statement on effectiveness of extending the profitable season. The rating scale for each statement was as follows: (4) Successful, (3) Moderately Successful, (2) Slightly Successful, (1) Unsuccessful. The tailored design methods of data collection by Dillman (2000) were followed in the second phase of the research study. The first step in the data collection process was to send a pre-card informing the subjects they would receive the questionnaire. The second step was to make the initial mailing of the packet including a cover letter, the instrument, and stamped self addressed envelope. Each envelope was coded for follow-up purposes only. Participants were asked to identify three strategies or practices used to extend the profitable season of their retail garden center. A follow-up postcard was sent to non- 
respondents after the initial return deadline. One week after the postcard, a second complete mailing was sent to non-respondents. A follow-up postcard was sent after the second deadline to non-respondents encouraging them to respond to the survey.

Non-response error was assessed by comparing early and late respondents because research has shown "non-respondents are often similar to late respondents" (Ary, 2006, p. 439). Respondents were categorized between early and late groups to compare their responses for any significant relationships. Using this comparison allows the researcher to reasonably assume that the respondents represent an unbiased sample of all who received the questionnaire. (Ary et al., 2006)

Analysis of Data

Each member of the population was assigned a numerical code prior to the initial mailing. This code enabled the researcher to document each survey received and identify non-respondents. The code was destroyed before the data was analyzed to protect the anonymity of the survey participant.

The collected data were analyzed using the Statistical Package for the Social Sciences (SPSS). An Excel spreadsheet was used for data entry. Each variable in the instrument was assigned a numerical value. Descriptive analyses including frequencies, percentages and means were used to evaluate numerical data to allow for conversion into narrative form.

\section{Use of Findings}

The findings of this study will be utilized to determine the current practices being subscribed by independently owned garden centers to extend the profitable season. The results will reflect the opinions of garden center managers on the effectiveness of these 
practices. The findings will be provided to participating garden centers and other appropriate groups. The results will also contribute to the body of knowledge on the continuing challenges facing the retail sector of the horticulture industry. 


\section{CHAPTER IV}

\section{Findings}

\section{Purpose of the Study}

The purpose of this study was to identify the measures that independently owned retail garden centers subscribe to extend their profitable season beyond the spring and early summer. By identifying profitable practices used by garden centers in the United States, the retail horticulture industry will benefit from increased profits and more employment opportunities. The entire green industry will also benefit from the increase in retail sales. The demand for plants, planting mediums and other garden accessories will have to increase to keep up with the retail sector. The manufacturing and production of plants, mediums and accessories will create more jobs and more money in the economy.

\section{Objectives of the Study}

The objectives for the study are reflected in the following research questions:

1. What measures are currently being practiced by garden centers to extend the profitable season beyond the spring and early summer?

2. What companion products do garden centers sell to extend the profitable season?

3. Which techniques used to extend the profitable season are viewed by retailers to be most helpful in raising profit margins?

4. Which techniques used to extend the profitable season are viewed by retailers to be most helpful in returning customers to the garden center? 
5. Which techniques are retailers most likely to incorporate in their current operation?

6. Is shutting down the garden center for an extended period of time a realistic option?

\section{Findings}

Phase I Questionnaire. The sample population $(\mathrm{N}=248)$ was independent retail garden centers that were members of the Ohio Floriculture Association as of November 2006 who resided in West Virginia, Ohio, Maryland, Pennsylvania, Virginia and Kentucky. A total of 34 completed surveys were returned for a $13.7 \%$ response rate.

The following statements were collected as a result of the first questionnaire and divided into three categories: Related to Horticulture, Not Related to Horticulture and Other.

Related to Horticulture:

- Container gardens for all seasons

- Larger sizes for annuals and perennials

- Garden services

- Showcase what is "showy" each week

- Build and decorate a straw house

- Fresh cut Christmas tree sales

- Hand-made fresh wreaths, swags, centerpieces

- Live pumpkin sales

- Fresh cut flower sales for Valentine's Day

- Have plants in stock when blooming in nature

- Focus on perennials not found at other stores

- Plants that can be used for weddings or outdoor gatherings

- Fall mixed containers using cool weather plants

- Blooming plant displays

- Extensive landscaped gardens

- Dormant pruning maintenance

- Multiple smaller crops to ensure longer fresh supply

- Less or no roses

- Diversification-florist, greenhouse, garden center, nursery 
- Growing your own products, allows for diverse product mix

- Landscape design and installation

- Cool weather annuals

- Herb topiaries

- Windowsill herb pots in stock in the fall

- Encourage fall planting of trees and shrubs using items in color at that time of year

- Custom greens decorating for Christmas

- Sell perennials that bloom in August or September

- Begin selling plants earlier in the season by stocking earlier in covered shopping areas

- Classes on preparing flower gardens for winter

- Classes on flowers that can be dried for indoor arrangements or wreaths

- Classes on drying and storing herbs for use in teas, infusions or for cooking.

- Force tulips, daffodils and other spring flowers for early sales

- Sell seed potatoes, garden seeds, garden fertilizers and soil amendments

- Fall festival: pony rides, food, entertainment

- Perennial party: sales contest with 2 teams at garden center (black eyed susans vs. coreopsis crushers

- Fall container program-pot your own-free soil

- Spring container program-pot your own-we'll grow it until Mother's Day-you pick it up

- Fall bulb seminar

- Canning supplies

Not Related to Horticulture:

- Expansion of hard goods

- Holiday sales

- Diversification of product lines that augment horticultural lifestyles

- Artificial Christmas Tree sales

- Ornaments, wreaths, outdoor displays, lights

- Snow blower sales

- Decorations for Easter

- Decorations for Thanksgiving

- Decorations for Halloween

- Decorations for Christmas

- Workshops/educational classes

- Participation in community events

- Autumn Fest with crafters, demonstrations by artisans

- Scare crow contest

- Petting zoo

- Corn maize

- Customer appreciation sale and celebration

- Snow removal 
- Seasonally dated coupons

- Percent off sale events

- Neat and clean grounds

- Greet customers as soon as possible

- Quality merchandise

- Knowledgeable, stable staff

- Community outreach

- School tours

- Pre-season mailing

- Company website

- Speaking at garden clubs

- Water gardening products

- Email new product updates to customers

- Wildlife/bird feeding supplies

- Gifts

- Pet food and supplies

- Sell gift certificates at $20 \%$ off in slowest months

- Spend $\$ 50$ get a free gift

- Offer extra stamps on loyalty cards in slowest months

- Custom Christmas decorating for customers

- Reduction of staff to match sales in the garden center

- Reduction of inventory levels during non-peak periods

- Raise prices on all plants

- Hire people who like people and not just selling plants

- Create a year round marketing program instead of seasonal

- Information on preparing outdoor furniture for winter conditions

- Classes on maintaining deck wood or furniture over winter

- How to maintain and preserve birdhouses and feeders

- Christmas decorating services: interior and exterior

- Offer local made crafts from area crafters

- Pot up bare root stock during winter months for spring sales

- Newsletters

- Website

- Farmers market and offer free space to vendors

- Arts and crafts show

- Christmas village: horse and buggy rides with Santa, hot chocolate

- Parking lot sale of giftware

- Free photos with Easter bunny and Santa clause

- Open house 
Other:

- Owning apartment buildings near garden center

- Only open from Easter until end of June

- Good location

- Having a covered sales area for comfortable shopping

- Renovating houses for resale

- Only open in the spring and early summer months

A panel of experts was used to compile the statements as well as statements from the literature review into ten categories of practices which participants could rate on the following scale: (4) Successful, (3) Moderately Successful, (2) Slightly Successful, (1) Unsuccessful.

Phase II Questionnaire. The sample population $(\mathrm{N}=248)$ for phase two was independent retail garden centers that are members of the Ohio Floriculture Association as of November 2006 residing in West Virginia, Ohio, Maryland, Pennsylvania, Virginia and Kentucky. A total of 32 completed surveys were returned, for a $12.9 \%$ response rate.

Ary, Jacobs, Razavieh \& Sorensen (2006) guiding principle to generalize the respondents to the entire population is to determine the degree to which respondents differ from non-respondents by comparing early and late respondents. Thirty-two surveys were returned completed, 16 were early respondents and 16 were late respondents. Chi-square tests were done on the variables level of education, gender, age and years of operation to compare similarities of early and late respondents. The chisquare value of level of education was $1.38(\mathrm{df}=3)$ and was not significant $(\alpha \leq .05)$. The chi-square value of gender was $1.77(\mathrm{df}=1)$ and was not significant $(\alpha \leq .05)$. The chi-square value of age was $2.35(\mathrm{df}=3)$ and was not significant $(\alpha \leq .05)$. The chisquare value of years of operation was .168 $(\mathrm{df}=4)$ and was not significant $(\alpha \leq .05)$. 
Research has shown "nonrespondents are often similar to late respondents" (Ary et al., 2006, p. 439). No significant difference was found between early and late respondents. Therefore, the findings could be generalized to the entire population of independent retail garden centers who were members of the Ohio Floriculture Association as of November 2006 in West Virginia, Ohio, Maryland, Pennsylvania, Virginia and Kentucky. Because of the low response rate, the researcher elected to limit generalizations to the garden centers who responded to the survey.

\section{Demographic Characteristics of Respondents}

Demographic data indicate that 19 of the respondents (59.4\%) were male, while $13(40.6 \%)$ were female. The highest level of education of 14 respondents $(43.8 \%)$ was high school diploma while the highest level of 14 respondents (43.8\%) was a bachelors degree. Three respondents $(9.3 \%)$ held an associates degree while one of the respondents (3.1\%) had a two year post secondary degree. Six of the respondents (18.8\%) were between the ages of 30-39 years. Nine respondents (28.1\%) were between the ages of 40-49 years and 11 respondents (34.3\%) were 50-59 years old. The remaining six respondents (18.8\%) were 60-69 years old. Of the respondents two $(6.3 \%)$ were certified horticulturists and 30 (93.7\%) were not (see Table 1). 
Table 1

Demographic Characteristics of Respondents

N $\%$

Gender

Male

19

$59.4 \%$

Female

13

$40.6 \%$

Level of Education

High School Diploma

Bachelors Degree

Associates Degree

Two Year Post Secondary Degree

Age

30-39 years

6

40-49 years

50-59 years

60-69 years

Certified Horticulturist

Yes

No

9

11

6

2

30
14

14

3

1
$3.1 \%$

$43.8 \%$

$43.8 \%$

$9.3 \%$

$18.8 \%$

$28.1 \%$

$34.3 \%$

$18.8 \%$

$6.3 \%$

$93.7 \%$

The number of years of operation for all garden centers were identified by the respondents. Three of the respondents $(9.4 \%)$ had been in operation less than five years. Five of the respondents (15.5\%) had been open for 6-10 years while two garden centers 
(6.3\%) had been in operation for 11-15 years. Two (6.3\%) garden centers had been in operation for $16-20$ years and 20 of the respondents $(62.5 \%)$ had been operating for 20 or more years (see Table 2).

Table 2

Years of Operation

\begin{tabular}{lcc}
\hline & $N$ & $\%$ \\
\hline Less than 5 years & 3 & 9.4 \\
6-10 years & 5 & 15.5 \\
$11-15$ years & 2 & 6.3 \\
$16-20$ years & 2 & 6.3 \\
20 or more years & 20 & 62.5 \\
\hline
\end{tabular}

Respondents were asked to identify the dates of operation of their business. Eighteen garden centers $(56.3 \%)$ were open year round. Two garden centers $(6.3 \%)$ were open during the spring and summer and closed in the fall and winter. Twelve respondents (37.4\%) listed other dates of operation (see Table 3). 
Table 3

Dates of Operation

\begin{tabular}{llc}
\hline & $N$ & $\%$ \\
\hline Open year round & 18 & 56.3 \\
& & 6.3 \\
Open spring and summer; closed fall and winter & 2 & 37.4 \\
Others (listed below) & 12 & \\
& & 25.1 \\
Spring, Summer, Fall & 3 & 16.7 \\
March-Thanksgiving & 2 & 16.7 \\
Closed January \& February only & 2 & 8.3 \\
Spring to Christmas & 1 & 8.3 \\
Closed Jan-April & 1 & 8.3 \\
Spring, Summer, Nov-Dec & 1 & 8.3 \\
March-November & 1 & \\
April, May, June \& Nov-Dec & 1 & \\
& & \\
\end{tabular}

Respondents were asked whether or not garden centers as a whole should close during the winter and only be open for the spring and summer months. Nineteen garden centers $(65.5 \%)$ said no to closing for the winter. Ten respondents $(34.5 \%)$ said yes garden centers should be closed during the winter (see Table 4).

Table 4

Seasonal Operation

\begin{tabular}{lcccc}
\hline & \multicolumn{2}{c}{ Yes } & \multicolumn{2}{c}{ No } \\
\cline { 2 - 5 } & $N$ & $\%$ & $N$ & $\%$ \\
\hline Spring-summer Operation & 10 & 34.5 & 19 & 65.5 \\
\hline
\end{tabular}

The square footage of the garden centers responding to the questionnaire was identified. Responding garden centers had an average indoor square footage of 15,790.32 
with a standard deviation of $21,157.78$ square feet. The average outdoor square footage of the respondents was $42,509.50$ with a standard deviation of 51,178.92 square feet. The minimum indoor square footage for the respondents was 800 square feet and the maximum was 80,000 square feet. The minimum outdoor square footage was zero and the maximum was 200,000 square feet (see Table 5).

Table 5

Square Footage of Garden Center

\begin{tabular}{lcccc}
\hline & Minimum & Maximum & $M$ & SD \\
\cline { 2 - 5 } Square Footage - Indoor & 800 & 80,000 & $15,790.32$ & $21,157.78$ \\
Square Footage - Outdoor & 0 & 200,000 & $42,509.50$ & $51,178.92$ \\
\hline
\end{tabular}

\section{Hours of Operation}

Twenty of the respondents (64.5\%) opened at 9:00 AM Monday through Friday. Three respondents $(9.7 \%)$ opened their stores at 8:00 AM, three stores $(9.7 \%)$ opened at 8:30 AM and another three (9.7\%) opened at 10:00 AM Monday through Friday. Two garden centers $(6.4 \%)$ responding to the questionnaire opened at 7:00 AM Monday through Friday.

Nineteen garden centers (61.3\%) opened their doors at 9:00 AM on Saturdays and five garden centers (16.1\%) opened at 8:00 AM. Three garden centers (9.7\%) open at 8:30 AM on Saturdays. Two of the respondents (6.5\%) opened at 7:00 AM on Saturdays and two (6.4\%) opened at 10:00 AM.

Eight garden centers $(33.3 \%)$ opened at noon on Sundays while seven garden centers (29.1\%) opened at 10:00 AM. Five of the responding garden centers $(20.8 \%)$ 
opened their doors at 9:00 AM on Sundays and two garden centers $(8.3 \%)$ open at 11:00 AM. The remaining two garden centers $(8.3 \%)$ opened at 1:00 PM (see Table 6).

Table 6

Opening Hour

\begin{tabular}{lcccccc}
\hline & \multicolumn{2}{c}{ Open Monday-Friday } & \multicolumn{2}{c}{ Open-Saturday } & \multicolumn{2}{c}{ Open-Sunday } \\
\cline { 2 - 7 } & $N$ & $\%$ & $N$ & $\%$ & $N$ & $\%$ \\
\hline 7:00 AM & 2 & 6.4 & 2 & 6.4 & 0 & 0.0 \\
8:00 AM & 3 & 9.7 & 5 & 16.1 & 0 & 0.0 \\
8:30 AM & 3 & 9.7 & 3 & 9.7 & 0 & 0.0 \\
9:00 AM & 20 & 64.5 & 19 & 61.3 & 5 & 20.8 \\
10:00 AM & 3 & 9.7 & 2 & 6.5 & 7 & 29.1 \\
11:00 AM & 0 & 0.0 & 0 & 0.0 & 2 & 8.3 \\
12:00 PM & 0 & 0.0 & 0 & 0.0 & 8 & 33.3 \\
1:00 PM & 0 & 0.0 & 0 & 0.0 & 2 & 8.3 \\
\hline
\end{tabular}

Of the garden centers responding to the questionnaire two $(6.4 \%)$ closed at 5:00 PM Monday through Friday. Fifteen of the respondents (48.4\%) closed at 6:00 PM. Eight of the garden centers (25.8\%) closed at 7:00 PM and the remaining six (19.4\%) closed at 8:00 PM.

Of the garden centers responding to the questionnaire four $(12.8 \%)$ closed their stores at 4:00 PM on Saturdays. Thirteen garden centers (41.9\%) closed at 5:00 PM and 10 garden centers $(32.3 \%)$ closed at 6:00 PM. Two garden centers $(6.5 \%)$ closed at 7:00 PM and another two (6.5\%) closed at 8:00 PM. 
One respondent (4.1\%) closed at 3:00 PM on Sundays. Four garden centers (16.7\%) closed at 4:00 PM and 13 garden centers (54.2\%) closed at 5:00 PM. The other six respondents $(25.0 \%)$ closed their garden centers at 6:00 PM (see Table 7).

\section{Table 7}

Closing Hour

\begin{tabular}{lcccccc}
\hline & \multicolumn{2}{c}{ Close Monday-Friday } & \multicolumn{2}{c}{ Close-Saturday } & \multicolumn{2}{c}{ Close-Sunday } \\
& $N$ & $\%$ & $N$ & $\%$ & $N$ & $\%$ \\
\cline { 2 - 6 } & 0 & 0.0 & 0 & 0.0 & 1 & 4.1 \\
4:00 PM & 0 & 0.0 & 4 & 12.8 & 4 & 16.7 \\
5:00 PM & 2 & 6.4 & 13 & 41.9 & 13 & 54.2 \\
6:00 PM & 15 & 48.4 & 10 & 32.3 & 6 & 25.0 \\
7:00 PM & 8 & 25.8 & 2 & 6.5 & 0 & 0.0 \\
8:00 PM & 6 & 19.4 & 2 & 6.5 & 0 & 0.0 \\
\hline
\end{tabular}

Based upon the opening and closing hours reported by garden centers, the total amount of hours open was calculated. Ten garden centers $(32.3 \%)$ were open for nine hours and six garden centers $(19.4 \%)$ were open for 10 hours a day. Five garden centers (16.1\%) were open for eight hours a day Monday through Friday. Four garden centers $(12.9 \%)$ were open for 11 hours while three garden centers $(9.7 \%)$ were open for 12 hours a day. Two garden centers $(6.4 \%)$ were open for 10.5 hours while one garden center $(3.2 \%)$ was open for a total of 11.5 hours a day.

Ten garden centers $(32.3 \%)$ were open for nine hours on Saturdays while nine garden centers $(29.0 \%)$ were open for 8 hours. Five garden centers $(16.1 \%)$ were open 
for 10 hours while three garden centers $(9.7 \%)$ were open for a total of seven hours on Saturdays. Two garden centers (6.4\%) were open for 8.5 hours. One garden center (3.2\%) was open for 11 hours and one garden center (3.2\%) was open for 11.5 hours on Saturday.

On Sundays, five garden centers (20.8\%) were open for five hours and another five garden centers $(20.8 \%)$ were open for six hours. Four garden centers $(16.6 \%)$ were open for eight hours. Three garden centers $(12.5 \%)$ were open for seven hours while three garden centers $(12.5 \%)$ were open for nine hours on Sundays. Two garden centers (8.3\%) were open for three hours and two garden centers (8.3\%) were open for four hours (see Table 8). 
Table 8

Hours Open Daily

\begin{tabular}{|c|c|c|c|c|c|c|}
\hline & \multicolumn{2}{|c|}{ Monday-Friday } & \multicolumn{2}{|c|}{ Saturdays } & \multicolumn{2}{|c|}{ Sundays } \\
\hline Hours & $N$ & $\%$ & $N$ & $\%$ & $N$ & $\%$ \\
\hline 3.0 & 0 & 0.0 & 0 & 0.0 & 2 & 8.3 \\
\hline 4.0 & 0 & 0.0 & 0 & 0.0 & 2 & 8.3 \\
\hline 5.0 & 0 & 0.0 & 0 & 0.0 & 5 & 20.8 \\
\hline 6.0 & 0 & 0.0 & 0 & 0.0 & 5 & 20.8 \\
\hline 7.0 & 0 & 0.0 & 3 & 9.7 & 3 & 12.5 \\
\hline 8.0 & 5 & 16.1 & 9 & 29.0 & 4 & 16.6 \\
\hline 8.5 & 0 & 0.0 & 2 & 6.4 & 0 & 0.0 \\
\hline 9.0 & 10 & 32.3 & 10 & 32.3 & 3 & 12.5 \\
\hline 10.0 & 6 & 19.4 & 5 & 16.1 & 0 & 0.0 \\
\hline 10.5 & 2 & 6.4 & 0 & 0.0 & 0 & 0.0 \\
\hline 11.0 & 4 & 12.9 & 1 & 3.2 & 0 & 0.0 \\
\hline 11.5 & 1 & 3.2 & 1 & 3.2 & 0 & 0.0 \\
\hline 12.0 & 3 & 9.7 & 0 & 0 & 0 & 0.0 \\
\hline
\end{tabular}

Practices Garden Centers Are Currently Using

Respondents were asked to express whether or not they were currently using practices listed in the questionnaire at their garden centers. The practices were divided into ten categories. Participants were also given the chance to write in other practices not listed in the questionnaire. 
Holiday Merchandise or Services. Twenty-two garden centers (71.0\%) were selling live pumpkins. Halloween decorations were being sold in 21 garden centers. Twenty-one garden centers (65.6\%) offered custom made decorations (e. g. wreaths, swags). Nineteen garden centers (61.3\%) were selling Christmas decorations. Garden centers selling container trees totaled 19 (59.4\%). Christmas gift plants (e. g. poinsettias) were sold in $53.1 \%$ of garden centers $(\mathrm{n}=17)$.

Sixteen respondents (50.0\%) said they were currently selling Easter decorations. Balled and burlap trees were being offered to customers by 15 garden centers (48.4\%). Easter gift plants (e. g. Easter lilies) were sold in 15 garden centers (46.9\%). Fifteen respondents $(46.9 \%)$ said they were currently selling Thanksgiving decorations. Freshcut Christmas trees were being sold in 14 of the responding garden centers $(43.8 \%)$. Ten garden centers (31.3\%) sold painted poinsettias to customers. Eight garden centers (25.0\%) sold artificial Christmas trees. Fresh cut Flowers for Valentine's Day were offered by five garden centers (15.6\%). Interior Christmas decorating services were offered by five garden centers (15.6\%). Exterior Christmas decorating services were offered by three garden centers $(9.4 \%)$ (see Table 9$)$. 
Table 9

Practices Garden Centers Are Currently Using_Holiday Merchandise or Services

\begin{tabular}{|c|c|c|c|c|}
\hline & \multicolumn{2}{|c|}{ Yes } & \multicolumn{2}{|c|}{ No } \\
\hline & $N$ & $\%$ & $N$ & $\%$ \\
\hline Live pumpkins & 22 & 71.0 & 9 & 29.0 \\
\hline Halloween decorations & 21 & 65.6 & 11 & 34.4 \\
\hline Custom made decorations (e. g. wreaths, swags) & 21 & 65.6 & 11 & 34.4 \\
\hline Christmas decorations & 19 & 61.3 & 12 & 38.7 \\
\hline Container trees & 19 & 59.4 & 13 & 40.6 \\
\hline Christmas gift plants (e. g. poinsettias) & 17 & 53.1 & 15 & 46.9 \\
\hline Easter decorations & 16 & 50.0 & 16 & 50.0 \\
\hline Balled and burlap trees & 15 & 48.4 & 16 & 51.6 \\
\hline Easter gift plants (e. g. Easter lilies) & 15 & 46.9 & 17 & 53.1 \\
\hline Thanksgiving decorations & 15 & 46.9 & 17 & 53.1 \\
\hline Fresh-cut Christmas trees & 14 & 43.8 & 18 & 56.3 \\
\hline Painted poinsettias & 10 & 31.3 & 22 & 68.8 \\
\hline Artificial Christmas trees & 8 & 25.0 & 24 & 75.0 \\
\hline Fresh cut flowers for Valentine's Day & 5 & 15.6 & 27 & 84.4 \\
\hline Interior Christmas decorating services & 5 & 15.6 & 27 & 84.4 \\
\hline Exterior Christmas decorating services & 3 & 9.4 & 29 & 90.6 \\
\hline
\end{tabular}

Children's Activities. Eleven garden centers (34.4\%) allow children to plant their own plants to take home. Pony rides were offered by four garden centers $(12.5 \%)$. 
Photos with Santa were offered by four garden centers (12.5\%). Four garden centers (12.5\%) had petting zoos for customers. Two garden centers (6.3\%) offered photos with the Easter Bunny to customers. Two garden centers (6.3\%) offered a corn maze for customers. A haunted greenhouse was presented by two garden centers (6.3\%). Two garden centers (6.3\%) built and decorated a straw house. None of the garden centers (100.0\%) sold Easter pets (e. g. chicks, ducks, bunnies) (see Table 10).

Table 10

Practices Garden Centers Are Currently Using—Children’s Activities

\begin{tabular}{lrrrr}
\hline & \multicolumn{3}{c}{ Yes } & \multicolumn{3}{c}{ No } \\
\cline { 2 - 6 } & $N$ & $\%$ & $N$ & $\%$ \\
\hline Plant their own plants to take home & 11 & 34.4 & 21 & 65.6 \\
Pony rides & 4 & 12.5 & 28 & 87.5 \\
Photos with Santa & 4 & 12.5 & 28 & 87.5 \\
Petting zoo & 4 & 12.5 & 28 & 87.5 \\
Photos with Easter Bunny & 2 & 6.3 & 30 & 93.8 \\
Corn maze & 2 & 6.3 & 30 & 93.8 \\
Haunted greenhouse & 2 & 6.3 & 30 & 93.8 \\
Build and decorate a straw house & 2 & 6.3 & 30 & 93.8 \\
Sell Easter pets (e. g. chicks, ducks, bunnies) & 0 & 0.0 & 32 & 100.0 \\
\hline
\end{tabular}

Advertisement. The newspaper was used by 25 garden centers (78.1\%) as advertisement. Twenty respondents (62.5\%) utilized a company website. Nineteen garden centers (59.4\%) used the radio as a method of advertisement. Fourteen garden 
centers $(45.2 \%)$ sent periodic newsletters to customers. Fourteen of the garden centers (45.2\%) used television as a method of advertisement. Ten garden centers $(34.5 \%)$ recommended using a year round marketing program instead of a seasonal program. Six garden centers $(19.4 \%)$ used emailing customers new product updates as a way to advertise (see Table 11).

Table 11

Practices Garden Centers Are Currently Using--Advertisement

\begin{tabular}{lcccc}
\hline & \multicolumn{2}{c}{ Yes } & \multicolumn{2}{c}{ No } \\
\cline { 2 - 5 } & $N$ & $\%$ & 7 & 21.9 \\
\hline Newspaper & 25 & 78.1 & 7 & 37.5 \\
Utilizing a company website & 20 & 62.5 & 12 & 40.6 \\
Radio & 19 & 59.4 & 13 & 54.8 \\
Sending periodic newsletters to customers & 14 & 45.2 & 17 & 54.8 \\
Television & 14 & 45.2 & 17 & \\
Using a year round marketing program instead of & & & & \\
seasonal & 10 & 34.5 & 19 & 65.5 \\
Emailing customers new product updates & 6 & 19.4 & 25 & 80.6 \\
\hline
\end{tabular}

Educational Seminars. Respondents were asked if their garden centers provided the following educational seminars for their customers. Fourteen garden centers $(45.2 \%)$ gave seminars on gardening basics to customers. Twelve garden centers $(40.0 \%)$ educated customers on planting. Eleven garden centers $(36.7 \%)$ educated customers on plants that attract birds and plants that attract butterflies. Pruning was a topic offered by 10 garden centers $(33.3 \%)$. Ten garden centers $(32.3 \%)$ educated customers on cooking 
with herbs. Dividing perennials was a seminar topic at nine garden centers (31.0\%). Eight garden centers (25.8\%) held seminars on preparing gardens for winter. Seven garden centers (23.3\%) educated customers on plants that repel pests (e. g. deer, rabbits, etc.). The dos and don'ts of mulch was a seminar topic at six garden centers (19.4\%). Customers were offered seminars on flower arranging at five garden centers (16.7\%). Seminars on drying and storing herbs were held in five of the responding garden centers (16.7\%). Four garden centers (13.3\%) educated customers on identifying diseases. Herbal health remedies was a topic for seminars at four garden centers (13.3\%). Three garden centers $(10.3 \%)$ held seminars on drying flowers. Identifying insect pests was a seminar topic at three garden centers $(10.0 \%)$. Gardening with out pain was taught at one garden center (3.4\%). Maintaining deck wood, maintaining outdoor furniture, preserving birdhouses and feeders and using landscape lighting to accent gardens at night were not used as seminar topics in any responding garden centers (100.0\%) (see Table 12). 
Table 12

Practices Garden Centers Are Currently Using-Educational Seminars

\begin{tabular}{|c|c|c|c|c|}
\hline & \multicolumn{2}{|c|}{ Yes } & \multicolumn{2}{|c|}{ No } \\
\hline & $N$ & $\%$ & $N$ & $\%$ \\
\hline Gardening basics & 14 & 45.2 & 17 & 54.8 \\
\hline Planting & 12 & 40.0 & 18 & 60.0 \\
\hline Plants that attract birds & 11 & 36.7 & 19 & 63.3 \\
\hline Plants that attract butterflies & 11 & 36.7 & 19 & 63.3 \\
\hline Pruning & 10 & 33.3 & 20 & 66.7 \\
\hline Cooking with herbs & 10 & 32.3 & 21 & 67.7 \\
\hline Dividing perennials & 9 & 31.0 & 20 & 69.0 \\
\hline Preparing gardens for winter & 8 & 25.8 & 23 & 74.2 \\
\hline Plants that repel pests (e. g. deer, rabbits, etc) & 7 & 23.3 & 23 & 76.7 \\
\hline Dos and don'ts of mulch & 6 & 19.4 & 25 & 80.6 \\
\hline Flower arranging & 5 & 16.7 & 25 & 83.3 \\
\hline Drying and storing herbs & 5 & 16.7 & 25 & 83.3 \\
\hline Identifying diseases & 4 & 13.3 & 26 & 86.7 \\
\hline Herbal health remedies & 4 & 13.3 & 26 & 86.7 \\
\hline Drying flowers & 3 & 10.3 & 26 & 89.7 \\
\hline Identifying insect pests & 3 & 10.0 & 27 & 90.0 \\
\hline Gardening without pain & 1 & 3.4 & 28 & 96.6 \\
\hline Using landscape lighting to accent gardens at night & 0 & 0.0 & 31 & 100.0 \\
\hline Preserving birdhouses and feeders & 0 & 0.0 & 30 & 100.0 \\
\hline Maintaining outdoor furniture & 0 & 0.0 & 31 & 100.0 \\
\hline Maintaining deck wood & 0 & 0.0 & 31 & 100.0 \\
\hline
\end{tabular}


Services for the Community. Twenty-four garden centers (75.0\%) donated plants to public areas. Twenty-one garden centers (65.6\%) donated supplies (e. g. mulch) to public areas. Five garden centers (16.7\%) offered a local craft show to customers. Three garden centers $(10.0 \%)$ offered farmers market with free spaces for vendors to the community. Farmers market with paid space for vendors was offered by one garden center (3.3\%) (see Table 13).

Table 13

Practices Garden Centers Are Currently Using-Services for the Community

\begin{tabular}{lrrrr}
\hline & \multicolumn{3}{c}{ Yes } & \multicolumn{2}{c}{ No } \\
\cline { 2 - 6 } & $N$ & $\%$ & $N$ & $\%$ \\
\hline Donation of plants to public areas & 24 & 75.0 & 8 & 25.0 \\
Donation of supplies to public areas (e. g. mulch) & 21 & 65.6 & 11 & 34.4 \\
Local craft show & 5 & 16.7 & 25 & 83.3 \\
Farmers market with free space for vendors & 3 & 10.0 & 27 & 90.0 \\
Farmers market with paid space for vendors & 1 & 3.3 & 29 & 96.7 \\
\hline
\end{tabular}

Services for Customers. Twenty-three garden centers (71.9\%) custom designed containers (e. g. hanging baskets, patio planters) for. Twenty-one garden centers $(65.6 \%)$ offered landscape design services to customers. Landscape installation was offered as a service at 20 garden centers $(62.5 \%)$. Sixteen garden centers (50.0\%) allowed customers to design containers that the greenhouse would grow until a pickup date. Fourteen garden centers (43.8\%) offered pruning shrubs as a service to customers. Nine garden centers $(29.0 \%)$ offered water garden design for customers. Eight garden centers $(25.0 \%)$ 
offered rental plants for weddings. Pruning trees was offered as a customer service at seven garden centers $(21.9 \%)$. Five garden centers $(16.1 \%)$ installed water gardens for customers. Floral design for weddings (e. g. corsages) was offered as a service by five garden centers $(15.6 \%)$. Two garden centers $(6.3 \%)$ offered lawn equipment repair as a service to customers. One garden center (3.1\%) offered rental plants for offices (see Table 14).

Table 14

Practices Garden Centers Are Currently Using-Services for Customers

\begin{tabular}{|c|c|c|c|c|}
\hline & \multicolumn{2}{|c|}{ Yes } & \multicolumn{2}{|c|}{ No } \\
\hline & $N$ & $\%$ & $N$ & $\%$ \\
\hline $\begin{array}{l}\text { Custom container design (e. g. hanging baskets, patio } \\
\text { planters) }\end{array}$ & 23 & 71.9 & 9 & 28.1 \\
\hline Landscape design & 21 & 65.6 & 11 & 34.4 \\
\hline Landscape installation & 20 & 62.5 & 12 & 37.5 \\
\hline $\begin{array}{l}\text { Customer designed containers - greenhouse grows } \\
\text { until pickup date }\end{array}$ & 16 & 50.0 & 16 & 50.0 \\
\hline Pruning shrubs & 14 & 43.8 & 18 & 56.3 \\
\hline Water garden design & 9 & 29.0 & 22 & 71.0 \\
\hline Rental plants for weddings & 8 & 25.0 & 24 & 75.0 \\
\hline Pruning trees & 7 & 21.9 & 25 & 78.1 \\
\hline Water garden installation & 5 & 16.1 & 26 & 83.9 \\
\hline Floral design for weddings (e. g. corsages) & 5 & 15.6 & 27 & 84.4 \\
\hline Lawn equipment repair & 2 & 6.3 & 30 & 93.7 \\
\hline Rental plants for offices & 1 & 3.1 & 31 & 96.9 \\
\hline
\end{tabular}


Lines of Plant Material. Summer blooming perennials were sold in 29 garden centers (90.6\%). Twenty-eight garden centers (87.5\%) offered perennials to customers. Summer annuals (e. g. wave petunias) were offered by 27 garden centers (84.4\%). Cool weather annuals (e. g. pansies) were offered to customers in 25 garden centers $(78.1 \%)$. Fall blooming ornamental grasses were offered by 25 garden centers $(78.1 \%)$. Twentytwo garden centers (68.8\%) offered larger sizes of annuals to customers. Twenty-four garden centers (75.0\%) offered larger sizes of perennials to customers. Outdoor container gardens were sold at 24 garden centers $(75.0 \%)$. Nineteen garden centers (59.4\%) sold windowsill herb pots to customers. House plants (e. g. African violets, ivy, etc) were sold in 18 garden centers $(56.3 \%)$. Seventeen garden centers $(53.1 \%)$ offered a line of plant propagation supplies (e. g. seed potatoes, onion sets and garden seeds) to customers. Sixteen garden centers (50.0\%) stocked fall bulbs (e. g. tulips). Tropical bulbs (e. g. caladium) were a line of merchandise in 14 garden centers (43.8\%). Spring bulbs (e. g. dahlias) were sold in 13 garden centers (41.9\%). Orchids were sold in 11 garden centers (35.5\%). Herb topiaries were stocked by 11 garden centers (34.4\%). Eight garden centers (25.0\%) provided container evergreens for indoors as a selection for customers. Four garden centers (13.3\%) sold fresh cut flowers year round (see Table 15). 
Table 15

Practices Garden Centers Are Currently Using_Lines of Plant Material

\begin{tabular}{|c|c|c|c|c|}
\hline & \multicolumn{2}{|c|}{ Yes } & \multicolumn{2}{|c|}{ No } \\
\hline & $N$ & $\%$ & $N$ & $\%$ \\
\hline Summer blooming perennials & 29 & 90.6 & 3 & 9.4 \\
\hline Perennials & 28 & 87.5 & 4 & 12.5 \\
\hline Summer annuals (e. g. wave petunias) & 27 & 84.4 & 5 & 15.6 \\
\hline Cool weather annuals (e. g. pansies) & 25 & 78.1 & 7 & 21.9 \\
\hline Fall blooming ornamental grasses & 25 & 78.1 & 7 & 21.9 \\
\hline Larger sizes of annuals & 22 & 68.8 & 10 & 31.3 \\
\hline Larger sizes of perennials & 24 & 75.0 & 8 & 25.0 \\
\hline Outdoor container gardens & 24 & 75.0 & 8 & 25.0 \\
\hline Windowsill herb pots & 19 & 59.4 & 13 & 40.6 \\
\hline House plants (e. g. African violets, ivy, etc) & 18 & 56.3 & 14 & 43.8 \\
\hline $\begin{array}{l}\text { Plant propagation supplies (e. g. seed potatoes, onion } \\
\text { sets and garden seeds) }\end{array}$ & 17 & 53.1 & 15 & 46.9 \\
\hline Fall bulbs (e. g. tulips) & 16 & 50.0 & 16 & 50.0 \\
\hline Tropical bulbs (e. g. caladium) & 14 & 43.8 & 18 & 56.2 \\
\hline Spring bulbs (e. g. dahlias) & 13 & 41.9 & 18 & 58.1 \\
\hline Orchids & 11 & 35.5 & 20 & 64.5 \\
\hline Herb topiaries & 11 & 34.4 & 21 & 65.6 \\
\hline Container evergreens for indoors & 8 & 25.0 & 24 & 75.0 \\
\hline Fresh cut flowers year round & 4 & 13.3 & 26 & 86.7 \\
\hline
\end{tabular}


Lines of Merchandise. Twenty-four garden centers (75.0\%) sold hard goods (e. g. pottery, statues, etc). Seventeen garden centers (56.7\%) offered hummingbird supplies to customers. Wind chimes were sold in 16 of the garden centers (50.0\%). Outdoor furniture and accessories were sold by 14 garden centers (45.2\%) that responded to the questionnaire. Garden flags were sold in 14 garden centers (43.8\%). Twelve garden centers (38.7\%) sold candles and accessories. Wildlife and/or bird feeding supplies were sold in 10 garden centers (32.3\%). Garden themed clothing was sold in five garden centers (16.1\%). Two garden centers (6.9\%) sold pet food and supplies to customers. Lawn equipment (e. g. mowers, weed trimmers) was sold by two garden centers (6.7\%). Two garden centers $(6.7 \%)$ sold local honey to customers. One garden center who responded (3.4\%) sold local wine. One garden center (3.3\%) sold other wine. Other honey was sold by one garden centers (3.3\%). Local jams and jellies were sold by one garden center (3.3\%). One garden center (3.3\%) sold other jams and jellies. One garden center (3.1\%) offered canning supplies to customers (see Table 16). 
Table 16

Practices Garden Centers Are Currently Using_Lines of Merchandise

\begin{tabular}{|c|c|c|c|c|}
\hline & \multicolumn{2}{|c|}{ Yes } & \multicolumn{2}{|c|}{ No } \\
\hline & $N$ & $\%$ & $N$ & $\%$ \\
\hline Hard goods (e. g. pottery, statues, etc) & 24 & 75.0 & 8 & 25.0 \\
\hline Hummingbird supplies & 17 & 56.7 & 13 & 43.3 \\
\hline Wind chimes & 16 & 50.0 & 16 & 50.0 \\
\hline Outdoor furniture and accessories & 14 & 45.2 & 17 & 54.8 \\
\hline Garden flags & 14 & 43.8 & 18 & 56.3 \\
\hline Candles and accessories & 12 & 38.7 & 19 & 61.3 \\
\hline Wildlife/bird feeding supplies & 10 & 32.3 & 21 & 67.7 \\
\hline Garden themed clothing & 5 & 16.1 & 26 & 83.9 \\
\hline Pet food and supplies & 2 & 6.9 & 27 & 93.1 \\
\hline Lawn equipment (e. g. mowers, weed trimmers) & 2 & 6.7 & 28 & 93.3 \\
\hline Local honey & 2 & 6.7 & 28 & 93.3 \\
\hline Local wine & 1 & 3.4 & 28 & 96.6 \\
\hline Other wine & 1 & 3.3 & 29 & 96.7 \\
\hline Other honey & 1 & 3.3 & 29 & 96.7 \\
\hline Local jams and jellies & 1 & 3.3 & 29 & 96.7 \\
\hline Other jams and jellies & 1 & 3.3 & 29 & 96.7 \\
\hline Canning supplies & 1 & 3.1 & 31 & 96.9 \\
\hline
\end{tabular}


Adjustments to Business or Location. Thirty garden centers (100.0\%) employed knowledgeable and experience staff as well as customer friendly staff (100.0\%). Twentyeight garden centers (93.3\%) kept their aisles and displays clean. Twenty-two garden centers $(75.9 \%)$ grew their own plants to allow for diverse product mix. Twenty-one garden centers (67.7\%) had established a warranty for plant material. Display gardens were a part of 18 garden centers (60.0\%). Prices had been raised on all items at 15 garden centers $(48.4 \%)$. Thirteen garden centers (43.3\%) added a covered shopping area for year round sales. Eleven garden centers (39.3\%) had established a "greeter" at the entrance of the store. Nine garden centers $(29.0 \%)$ had hired a certified horticulturist. Seven garden centers $(24.1 \%)$ had become bonded. Two garden centers $(6.7 \%)$ added a coffee house. One garden center (3.2\%) converted the greenhouse to a banquet hall during non-peak months. Thirty garden centers (100.0\%) did not have a bakery nor did they have an ice cream shop (see Table 17). 
Table 17

Practices Garden Centers Are Currently Using-Adjustments to Business or Location

\begin{tabular}{|c|c|c|c|c|}
\hline & \multicolumn{2}{|c|}{ Yes } & \multicolumn{2}{|c|}{ No } \\
\hline & $N$ & $\%$ & $N$ & $\%$ \\
\hline Knowledgeable and experienced staff & 30 & 100.0 & 0 & 0.0 \\
\hline Customer friendly staff & 30 & 100.0 & 0 & 0.0 \\
\hline Neat and clean aisles and displays & 28 & 93.3 & 2 & 6.7 \\
\hline Growing your own plants to allow for diverse product mix & 22 & 75.9 & 7 & 24.1 \\
\hline Establish warranty for plant material & 21 & 67.7 & 10 & 32.3 \\
\hline Display gardens & 18 & 60.0 & 12 & 40.0 \\
\hline Raise prices on all items & 15 & 48.4 & 16 & 51.6 \\
\hline Add a covered shopping area for year round sales & 13 & 43.3 & 17 & 56.7 \\
\hline Establish a "greeter" at entrance & 11 & 39.3 & 17 & 60.7 \\
\hline Hire a certified horticulturist & 9 & 29.0 & 22 & 71.0 \\
\hline Become bonded & 7 & 24.1 & 22 & 75.9 \\
\hline Add a coffee house & 2 & 6.7 & 28 & 93.3 \\
\hline $\begin{array}{l}\text { Convert greenhouse to banquet hall during non-peak } \\
\text { months }\end{array}$ & 1 & 3.2 & 30 & 96.8 \\
\hline Add a bakery & 0 & 0.0 & 30 & 100.0 \\
\hline Add an ice cream shop & 0 & 0.0 & 30 & 100.0 \\
\hline
\end{tabular}

Customer Appreciation. Twenty-two garden centers (71.0\%) held percent-off sale events for customers. Seasonally dated coupons were used by 18 garden centers $(60.0 \%)$ to appreciate customers. Nine garden centers $(30.0 \%)$ participated in a spend 
$\$ 50$ get free gift event. Eight garden centers (25.8\%) offered holiday specials on corresponding plants and accessories (i.e. Memorial Day: red, white and blue). Six garden centers (19.4\%) sold gift certificates at 20 percent off during non-peak months. Extra stamps were offered on loyalty cards in non-peak months by five garden centers (16.7\%). One garden center (3.2\%) offered specials to customers in costume on Halloween (see Table 18).

Table 18

Practices Garden Centers Are Currently Using-Customer Appreciation

\begin{tabular}{lcccc}
\hline & \multicolumn{3}{c}{ Yes } & \multicolumn{2}{c}{ No } \\
\cline { 2 - 6 } & $N$ & $\%$ & $N$ & $\%$ \\
\hline Percent-off sale events & 22 & 71.0 & 9 & 29.0 \\
Seasonally dated coupons & 18 & 60.0 & 12 & 40.0 \\
Spend \$ 50 get___free & 9 & 30.0 & 21 & 70.0 \\
$\begin{array}{l}\text { Holiday specials on corresponding plants and accessories } \\
\text { i.e. Memorial Day: red, white and blue }\end{array}$ & 8 & 25.8 & 23 & 74.2 \\
$\begin{array}{l}\text { Sell gift certificates at 20 percent off during non-peak } \\
\text { months }\end{array}$ & 6 & 19.4 & 25 & 80.6 \\
Offer extra stamps on loyalty cards in non-peak months & 5 & 16.7 & 25 & 83.3 \\
Offer specials to customers in costume on Halloween & 1 & 3.2 & 30 & 96.8 \\
\hline
\end{tabular}

Potential for Success in Getting Customers to Return to the Store

Respondents were asked to rate the practices on their potential for success in getting customers to return to the store. Respondents were to rate the practice on the following scale: (4) Successful, (3) Moderately Successful, (2) Slightly Successful, (1) 
Unsuccessful. The scale used in the research identified mean scores ranging from 3.264.0 as successful, 2.51-3.25 as moderately successful, 1.76-2.50 as slightly successful and $1.0-1.75$ as unsuccessful.

Holiday Merchandise or Services. Using a scale of 1-4 with 1 being "unsuccessful" and 4 "successful" the average rating of Christmas gift plants (e. g. poinsettias) was $3.15(S D=1.09)$ (see Table 19). Christmas gift plants were rated unsuccessful by two respondents (10.0\%). Four respondents (20.0\%) rated Christmas gift plants slightly successful in returning customers to the store while three respondents (15.0\%) rated Christmas gift plants moderately successful. Eleven respondents (55.0\%) rated Christmas gift plants successful in returning customers to the store.

The average rating of custom made decorations (e. g. wreaths, swags) was 3.00 $(S D=1.05)($ see Table 19). Custom made decorations were rated unsuccessful at returning customers to the store by two respondents $(9.5 \%)$. Five respondents $(23.8 \%)$ rated custom made decorations slightly successful while five respondents $(23.8 \%)$ rated them moderately successful. Nine respondents $(42.9 \%)$ rated custom made decorations successful in returning customers to the store.

The average rating of live pumpkins was $2.96(S D=.83)$ (see Table 19). Live pumpkins were rated unsuccessful by one respondent (4.4\%). Five respondents $(21.7 \%)$ rated live pumpkins slightly successful while 11 respondents (47.8\%) rated live pumpkins moderately successful in returning customers to the store. Six respondents (26.1\%) rated live pumpkins successful.

The average rating of Easter gift plants (e. g. Easter lilies) was $2.76(S D=.97)$ (see Table 19). Easter gift plants were rated unsuccessful by two respondents (11.8\%). 
Four respondents $(23.5 \%)$ rated Easter gift plants slightly successful at returning customers to the store while seven respondents (41.2\%) rated Easter gift plants moderately successful. Four respondents $(23.5 \%)$ rated Easter gift plants successful in returning customers to the store.

The average rating of painted poinsettias was $2.69(S D=1.25)$. Painted poinsettias were rated unsuccessful by three respondents $(23.1 \%)$. Three respondents $(23.1 \%)$ rated painted poinsettias slightly successful in returning customers to the store while two respondents $(15.4 \%)$ rated painted poinsettias moderately successful. Five respondents $(38.4 \%)$ rated painted poinsettias successful in returning customers to the store.

The average rating of Christmas decorations was $2.68(S D=1.13)$ (see Table 19). Five respondents $(22.7 \%)$ rated Christmas decorations unsuccessful in returning customers to the store. Three respondents (13.6\%) rated Christmas decorations slightly successful while eight respondents (36.4\%) rated Christmas decorations moderately successful. Christmas decorations were rated successful by six respondents $(27.3 \%)$.

The average rating of container trees was $2.68(S D=1.09)$ (see Table 19). Container trees were rated unsuccessful in returning customers to the store by three respondents (13.6\%). Eight respondents (36.4\%) rated container trees as slightly successful while four respondents $(18.2 \%)$ rated container trees moderately successful. Seven respondents $(31.8 \%)$ rated container trees successful at returning customers to the store.

The average rating of balled and burlap trees was $2.61(S D=1.09)$ (see Table 19). Three respondents $(16.7 \%)$ rated balled and burlap trees unsuccessful in returning 
customers to the store. Six respondents (33.3\%) rated balled and burlap trees slightly successful while four respondents $(22.2 \%)$ rated balled and burlap trees moderately successful. Balled and burlap trees were rated successful by five respondents $(27.8 \%)$.

The average rating of fresh-cut Christmas trees was $2.61(S D=1.04)$ (see Table 19). Fresh-cut Christmas trees were rated unsuccessful by three respondents (16.7\%). Five respondents $(27.8 \%)$ rated fresh-cut Christmas trees slightly successful in returning customers to the store while six respondents (33.3\%) rated fresh-cut Christmas trees moderately successful. Four respondents $(22.2 \%)$ rated fresh-cut Christmas trees successful.

The average rating of Halloween decorations was $2.59(S D=.85)$ (see Table 19). One respondent (4.5\%) rated Halloween decorations unsuccessful. Eleven respondents $(50.0 \%)$ rated Halloween decorations slightly successful while six respondents $(27.3 \%)$ moderately successful. Four respondents (18.2\%) rated Halloween decorations successful in returning customers to the store.

The average rating of fresh cut flowers for Valentine's Day was $2.57(S D=1.27)$ (see Table 19). Fresh cut flowers for Valentine's Day were rated unsuccessful by five respondents $(15.6 \%)$. Twenty seven respondents $(84.4 \%)$ rated fresh cut flower for Valentine's Day slightly successful in returning customers to the store.

The average rating of Easter decorations was $2.26(S D=.81)$ (see Table 19). Three respondents (15.7\%) rated Easter decorations as unsuccessful in returning customers to the store. Nine respondents (47.4\%) rated Easter decorations slightly successful while six respondents (31.6\%) rated Easter decorations moderately successful. One respondent (5.3\%) rated Easter decorations successful. 
The average rating of interior Christmas decorating services was $2.22(S D=1.30)$ (see Table 19). Interior Christmas decorating services were rated unsuccessful by four respondents $(44.5 \%)$. One respondent (11.1\%) rated interior Christmas decorating services as slightly successful while two respondents (22.2\%) rated them moderately successful. Two respondents (22.2\%) rated interior Christmas decorating services successful.

The average rating of Thanksgiving decorations was $2.17(S D=.62)$ (see Table 19). One respondent (5.6\%) rated Thanksgiving decorations as unsuccessful. Fourteen respondents (77.7\%) rated Thanksgiving decorations as slightly successful while two respondents (11.1\%) rated them moderately successful. One respondent (5.6\%) rated Thanksgiving decorations successful.

The average rating of artificial Christmas trees was $2.00(S D=1.16)$ (see Table 19). Six respondents (46.1\%) rated artificial Christmas trees unsuccessful at returning customers to the store. Three respondents $(23.1 \%)$ rated artificial trees slightly successful and two respondents (15.4\%) rated artificial trees moderately successful. Artificial Christmas trees were rated successful by two respondents (15.4\%).

The average rating of exterior Christmas decorating services was $1.88(S D=1.13)$ (see Table 19). Four respondents (50.0\%) rated exterior Christmas decorating services as unsuccessful in returning customers to the store. Two respondents (25.0\%) rated exterior Christmas decorating services slightly successful while one respondent (12.5\%) rated the service moderately successful. One respondent (12.5\%) rated exterior Christmas decorations successful at returning customers to the store (see Table 19). 
Table 19

Potential for Success in Getting Customers to Return to the Store-Holiday Merchandise or Services

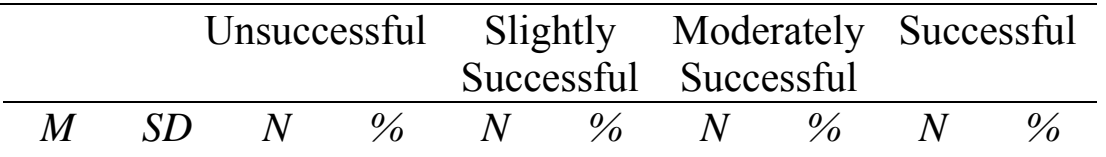

Christmas gift plants (e.

g. poinsettias)

$\begin{array}{llllllllll}3.15 & 1.09 & 32 & 10.0 & 4 & 20.0 & 3 & 15.0 & 11 & 55.0\end{array}$

Custom made

decorations (e. g.

wreaths, swags)

$\begin{array}{llllllllll}3.00 & 1.05 & 2 & 9.5 & 5 & 23.8 & 5 & 23.8 & 9 & 42.9\end{array}$

Live pumpkins

$\begin{array}{llllllllll}2.96 & .83 & 1 & 4.4 & 5 & 21.7 & 11 & 47.8 & 6 & 26.1\end{array}$

Easter gift plants (e. g.

Easter lilies)

$\begin{array}{llllllllll}2.76 & .97 & 2 & 11.8 & 4 & 23.5 & 7 & 41.2 & 4 & 23.5\end{array}$

$\begin{array}{lllllllllll}\text { Painted poinsettias } & 2.69 & 1.25 & 3 & 23.1 & 3 & 23.1 & 2 & 15.4 & 5 & 38.4\end{array}$

$\begin{array}{lllllllllll}\text { Christmas decorations } & 2.68 & 1.13 & 5 & 22.7 & 3 & 13.6 & 8 & 36.4 & 6 & 27.3\end{array}$

$\begin{array}{lllllllllll}\text { Container trees } & 2.68 & 1.09 & 3 & 13.6 & 8 & 36.4 & 4 & 18.2 & 7 & 31.8\end{array}$

$\begin{array}{lllllllllll}\text { Balled and burlap trees } & 2.61 & 1.09 & 3 & 16.7 & 6 & 33.3 & 4 & 22.2 & 5 & 27.8\end{array}$

Fresh cut Christmas

$\begin{array}{lllllllllll}\text { trees } & 2.61 & 1.04 & 3 & 16.7 & 5 & 27.8 & 6 & 33.3 & 4 & 22.2\end{array}$

$\begin{array}{lllllllllll}\text { Halloween decorations } & 2.59 & .85 & 1 & 4.5 & 11 & 50.0 & 6 & 27.3 & 4 & 18.2\end{array}$

Fresh cut flowers for

$\begin{array}{lllllllllll}\text { Valentine's Day } & 2.57 & 1.27 & 5 & 15.6 & 27 & 84.4 & 0 & 0.0 & 0 & 0.0\end{array}$

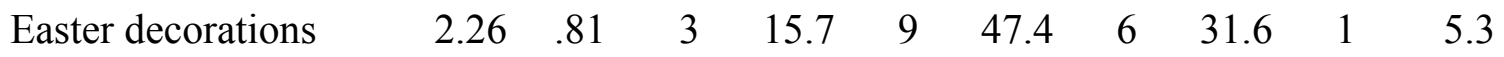

Interior Christmas

$\begin{array}{lllllllllll}\text { decorating services } & 2.22 & 1.30 & 4 & 44.5 & 1 & 11.1 & 2 & 22.2 & 2 & 22.2\end{array}$

Thanksgiving

decorations

$\begin{array}{llllllllll}2.17 & .62 & 1 & 5.6 & 14 & 77.7 & 2 & 11.1 & 1 & 5.6\end{array}$

Artificial Christmas

trees

$\begin{array}{llllllllll}2.00 & 1.16 & 6 & 46.1 & 3 & 23.1 & 2 & 15.4 & 2 & 15.4\end{array}$

Exterior Christmas

\begin{tabular}{lllllllllll} 
decorating services & 1.88 & 1.13 & 4 & 50.0 & 2 & 25.0 & 1 & 12.5 & 1 & 12.5 \\
\hline
\end{tabular}


Children's Activities. Using a scale of 1-4 with 1 being "unsuccessful" and 4 "successful" the average rating of letting children plant their own plant to take home was $3.09(S D=1.04)$ (see Table 20). Letting children plant their own plants to take home was rated unsuccessful by one respondent $(9.1 \%)$. Two respondents $(18.2 \%)$ rated the practice slightly successful while three respondents $(27.3 \%)$ rated it moderately successful. Five respondents (45.4\%) rated letting children plant their own plants to take home successful in returning customers to the store.

The average rating of corn maze was $2.75(S D=1.26)$ (see Table 20). One respondent (25.0\%) rated a corn maze unsuccessful in returning customers to the store. Two respondents (50.0\%) rated a corn maze moderately successful while one respondent $(25.0 \%)$ rated it successful in returning customers to the store.

The average rating of petting zoo was $2.60(S D=1.34)$ (see Table 20). One respondent $(20.0 \%)$ rated a petting zoo unsuccessful in returning customers to the store. Two respondents $(40.0 \%)$ rated a petting zoo slightly successful while two respondents $(40.0 \%)$ rated a petting zoo successful.

The average rating of pony rides was $2.60(S D=1.34)$ (see Table 20). Pony rides were rated unsuccessful in returning customers to the store by four respondents $(12.5 \%)$. Twenty eight respondents (87.5\%) rated pony rides slightly successful in returning customers to the store (see Table 24).

The average rating of haunted greenhouse was $2.33(S D=1.53)$ (see Table 20). Hosting a haunted greenhouse was rated unsuccessful bye one respondent (33.3\%). One respondent (33.3\%) rated hosting a haunted greenhouse slightly successful in returning 
customers to the store. One respondent $(33.3 \%)$ rated hosting a haunted greenhouse successful.

The average rating of photos with Santa was $2.20(S D=.84)$ (see Table 20). Photos with Santa were rated unsuccessful by one respondent $(20.0 \%)$. Two respondents rated photos with Santa (40.0\%) slightly successful in returning customers to the store while two respondents $(40.0 \%)$ rated photos with Santa moderately successful.

The average rating of photos with the Easter Bunny was $2.00(S D=.82)$ (see Table 20). Photos with the Easter Bunny were rated unsuccessful by one respondent (25.0\%). Two respondents $(50.0 \%)$ rated photos with the Easter Bunny slightly successful in returning customers to the store while one respondent $(25.0 \%)$ rated photos with the Easter Bunny moderately successful.

The average rating of build and decorate a straw house was $1.50(S D=.71)$ (see Table 20). Building and decorating a straw house was rated unsuccessful by one respondent $(50.0 \%)$ while it was rated slightly successful by one respondent $(50.0 \%)$.

The average rating of sell Easter pets was $1.00(S D=.00)$ (see Table 20). Selling Easter pets (e. g. chicks, ducks, bunnies) was rated unsuccessful by two respondents (100.0\%). 
Table 20

Potential for Success in Getting Customers to Return to the Store-Children's Activities

\begin{tabular}{|c|c|c|c|c|c|c|c|c|c|c|}
\hline & & & succ & essful & $\begin{array}{l}\text { Sliq } \\
\text { Succ }\end{array}$ & $\begin{array}{l}\text { tly } \\
\text { ssful }\end{array}$ & $\begin{array}{l}\text { Moc } \\
\text { Suc }\end{array}$ & $\begin{array}{l}\text { rately } \\
\text { ssful }\end{array}$ & Suc & ssful \\
\hline & $M$ & $S D$ & $N$ & $\%$ & $N$ & $\%$ & $N$ & $\%$ & $N$ & $\%$ \\
\hline $\begin{array}{l}\text { Plant their own plants to } \\
\text { take home }\end{array}$ & 3.09 & 1.04 & 1 & 9.1 & 2 & 18.2 & 3 & 27.3 & 5 & 45.4 \\
\hline Corn maze & 2.75 & 1.26 & 1 & 25.0 & 0 & 0.0 & 2 & 50.0 & 1 & 25.0 \\
\hline Petting zoo & 2.60 & 1.34 & 1 & 20.0 & 2 & 40.0 & 0 & 0.0 & 2 & 40.0 \\
\hline Pony rides & 2.60 & 1.34 & 4 & 12.5 & 28 & 87.5 & 0 & 0.0 & 0 & 0.0 \\
\hline Haunted greenhouse & 2.33 & 1.53 & 1 & 33.3 & 1 & 33.3 & 0 & 0.0 & 1 & 33.3 \\
\hline Photos with Santa & 2.20 & .84 & 1 & 20.0 & 2 & 40.0 & 2 & 40.0 & 0 & 0.0 \\
\hline Photos with Easter Bunny & 2.00 & .82 & 1 & 25.0 & 2 & 50.0 & 1 & 25.0 & 0 & 0.0 \\
\hline $\begin{array}{l}\text { Build and decorate a straw } \\
\text { house }\end{array}$ & 1.50 & .71 & 1 & 50.0 & 1 & 50.0 & 0 & 0.0 & 0 & 0.0 \\
\hline $\begin{array}{l}\text { Sell Easter pets (e. g. } \\
\text { chicks, ducks, bunnies) }\end{array}$ & 1.00 & .00 & 2 & 100.0 & 0 & 0.0 & 0 & 0.0 & 0 & 0.0 \\
\hline
\end{tabular}

Advertisement. Using a scale of 1-4 with 1 being "unsuccessful" and 4

"successful" the average rating of television was $3.06(S D=.99)$ (see Table 21$)$.

Television was rated unsuccessful in returning customers to the store by 14 respondents (45.2\%). Seventeen respondents (54.8\%) rated television as slightly successful in returning customers to the store.

The average rating of using a year round marketing program instead of seasonal was $3.00(S D=1.00)$ (see Table 21$)$. Using a year round marketing program instead of a 
seasonal program was rated unsuccessful by one respondent $(9.0 \%)$. Two respondents $(18.2 \%)$ rated the practice slightly successful in returning customers to the store while four respondents $(36.4 \%)$ rated the practice moderately successful. Four respondents (36.4\%) rated using a year round marketing program successful.

The average rating of sending periodic newsletters to customers was $2.93(S D=$ .73) (see Table 21). Sending periodic newsletters to customers was rated slightly successful by four respondents $(28.6 \%)$. Seven respondents $(50.0 \%)$ rated the practice moderately successful and three respondents $(21.4 \%)$ rated the practice successful.

The average rating of utilizing a company website was $2.82(S D=.66)$ (see Table 21). Seven respondents (31.8\%) rated utilizing a company website to return customers to the store slightly successful. Twelve respondents $(54.5 \%)$ rated the practice moderately successful. Three respondents (13.7\%) rated utilizing a company website successful in returning customers to the store.

The average rating of newspaper was $2.80(S D=.91)$ (see Table 21). Three respondents $(12.0 \%)$ rated the newspaper unsuccessful in returning customers to the store. Four respondents (16.0\%) rated the newspaper slightly successful while 13 respondents $(52.0 \%)$ rated the newspaper moderately successful. Five respondents $(20.0 \%)$ rated the newspaper successful in returning customers to the store.

The average rating of radio was $2.80(S D=.77)$ (see Table 21$)$. One respondent $(5.0 \%)$ rated the radio unsuccessful in returning customers to the store. Five respondents $(25.0 \%)$ rated the radio slightly successful while 11 respondents $(55.0 \%)$ rated the practice moderately successful. Three respondents $(15.0 \%)$ rated the radio successful in returning customers to the store. 
The average rating of emailing customers new product updates was $2.63(S D=$ .92) (see Table 21). Emailing customers new product updates was rated unsuccessful by one respondent $(12.5 \%)$. Two respondents $(25.0 \%)$ rated email slightly successful in returning customers to the store. Four respondents $(50.0 \%)$ rated emailing customers moderately successful and one respondent (12.5\%) rated the practice successful (see

Table 21).

Table 21

Potential for Success in Getting Customers to Return to the Store-Advertisement

\begin{tabular}{|c|c|c|c|c|c|c|c|c|c|c|}
\hline & \multicolumn{4}{|c|}{ Unsuccessful } & \multicolumn{2}{|c|}{$\begin{array}{l}\text { Slightly } \\
\text { Successful }\end{array}$} & \multicolumn{4}{|c|}{$\begin{array}{l}\text { Moderately Successful } \\
\text { Successful }\end{array}$} \\
\hline & $M$ & $S D$ & $N$ & $\%$ & $N$ & $\%$ & $N$ & $\%$ & $N$ & $\%$ \\
\hline Television & 3.06 & .99 & 14 & 45.2 & 17 & 54.8 & 0 & 0.0 & 0 & 0.0 \\
\hline $\begin{array}{l}\text { Using a year round marketing } \\
\text { program instead of seasonal }\end{array}$ & 3.00 & 1.00 & 1 & 9.0 & 2 & 18.2 & 4 & 36.4 & 4 & 36.4 \\
\hline $\begin{array}{l}\text { Sending periodic newsletters } \\
\text { to customers }\end{array}$ & 2.93 & .73 & 0 & 0.0 & 4 & 28.6 & 7 & 50.0 & 3 & 21.4 \\
\hline Utilizing a company website & 2.82 & .66 & 0 & 0.0 & 7 & 31.8 & 12 & 54.5 & 3 & 13.7 \\
\hline Newspaper & 2.80 & .91 & 3 & 12.0 & 4 & 16.0 & 13 & 52.0 & 5 & 20.0 \\
\hline Radio & 2.80 & .77 & 1 & 5.0 & 5 & 25.0 & 11 & 55.0 & 3 & 15.0 \\
\hline $\begin{array}{l}\text { Emailing customers new } \\
\text { product updates }\end{array}$ & 2.63 & .92 & 1 & 12.5 & 2 & 25.0 & 4 & 50.0 & 1 & 12.5 \\
\hline
\end{tabular}


Educational Seminars. The following seminars were rated on their effectiveness in returning customers to the store. Using a scale of 1-4 with 1 being "unsuccessful" and 4 "successful" the average rating of flower arranging was $3.00(S D=.58)$ (see Table 22). Flower arranging was rated slightly successful by one respondent (14.3\%). Five respondents (71.4\%) rated the seminar moderately successful. One respondent (14.3\%) rated the seminar successful at returning customers to the store.

The average rating of dividing perennials was $2.78(S D=.83)$ (see Table 22). Dividing perennials was rated unsuccessful by one respondent (11.1\%) in returning customers to the store. One respondent (11.1\%) rated the seminar slightly successful while six respondents $(66.7 \%)$ rated the seminar moderately successful. Dividing perennials was rated successful by one respondent (11.1\%).

The average rating of cooking with herbs was $2.60(S D=.84)($ see Table 22$)$. Cooking with herbs was rated unsuccessful by one respondent (10.0\%). Three respondents (30.0\%) rated the seminar slightly successful while five respondents $(50.0 \%)$ rated the seminar moderately successful. One respondent (10.0\%) rated cooking with herbs successful.

The average rating of herbal health remedies was $2.60(S D=1.15)$ (see Table 22). The herbal health remedies seminar was rated unsuccessful by one respondent $(20.0 \%)$. One respondent (20.0\%) rated the seminar slightly successful while two respondents $(40.0 \%)$ rated the seminar moderately successful. One respondent $(20.0 \%)$ rated the herbal health remedies seminar successful in returning customers to the store.

The average rating of plants that attract butterflies was $2.55(S D=1.04)$ (see Table 22). Three respondents (27.3\%) rated seminars on plants that attract butterflies 
unsuccessful in returning customers to the store. Seven respondents $(63.6 \%)$ rated the seminar moderately successful while one respondent $(9.1 \%)$ rated the seminar successful.

The average rating of pruning was $2.55(S D=.82)($ see Table 22$)$. Pruning seminars were rated unsuccessful by one respondent $(9.1 \%)$. Four respondents $(36.3 \%)$ rated the seminar slightly successful while five respondents $(45.5 \%)$ rated it moderately successful. One respondent $(9.1 \%)$ rated the seminar successful at returning customers to the store.

The average rating of gardening basics was $2.54(\mathrm{SD}=.88)$ (see Table 22). Two respondents (15.4\%) rated a seminar on gardening basics as unsuccessful in returning customers to the store. Three respondents (23.1\%) rated the seminar slightly successful while seven respondents $(53.8 \%)$ rated the seminar moderately successful. One respondent $(7.7 \%)$ rated the seminar successful in returning customers to the store.

The average rating of planting was $2.54(S D=.78)$ (see Table 22). Planting seminars were rated unsuccessful at returning customers to the store by 12 respondents (40.0\%). Eighteen garden centers $(60.0 \%)$ rated the seminar slightly successful.

The average rating of drying and storing herbs was $2.50(S D=1.05)$ (see Table 22). Drying and storing herbs was rated unsuccessful by one respondent (16.7\%). Two respondents $(33.3 \%)$ rated the seminar slightly successful while two respondents $(33.3 \%)$ rated the seminar moderately successful. One respondent (16.7\%) rated drying and storing herbs successful in returning customers to the store.

The average rating of preparing gardens for winter was $2.50(S D=1.20)$ (see Table 22). Preparing gardens for winter was rated unsuccessful by two respondents $(25.0 \%)$. The seminar was rated slightly successful bye two respondents $(25.0 \%)$ while 
two respondents $(25.0 \%)$ rated it moderately successful. Two respondents also rated the seminar successful.

The average rating of plants that attract birds was $2.45(S D=1.04)$ (see Table 22). Seminars on plants that attract birds were rated unsuccessful by three respondents (27.3\%). One respondent $(9.1 \%)$ rated the seminar slightly successful while six respondents $(54.5 \%)$ rated it moderately successful. One respondent $(9.1 \%)$ rated the seminar successful in returning customers to the store.

The average rating of plants that repel pests (e. g. deer, rabbits, etc) was 2.43 (SD $=1.27$ ) (see Table 22). Seminars on plants that repel pests was rated unsuccessful bye two respondents $(28.6 \%)$. Two respondents $(28.6 \%)$ rated the seminar slightly successful while one respondent $(14.2 \%)$ rated the seminar moderately successful. Two respondents $(28.6 \%)$ rated the seminar successful at returning customers to the store.

The average rating of identifying insect pests was $1.80(S D=.84)$ (see Table 22). Two respondents $(40.0 \%)$ rated a seminar on identifying insect pests unsuccessful in returning customers to the store. Two respondents $(40.0 \%)$ rated the seminar slightly successful and one respondent $(20.0 \%)$ rated the seminar moderately successful.

The average rating of the dos and don'ts of mulch was $2.38(S D=1.06)$ (see Table 22). The dos and don'ts of mulch seminar was rated unsuccessful by two respondents $(25.0 \%)$. Two respondents $(25.0 \%)$ rated the seminar slightly successful while three respondents $(37.5 \%)$ rated it moderately successful. One respondent $(12.5 \%)$ rated the dos and don'ts of mulch seminar successful in returning customers to the store.

The average rating of identifying diseases was $2.00(S D=.89)$ (see Table 22). Two respondents $(33.3 \%)$ rated the identifying diseases seminar unsuccessful in returning 
customers to the store. Two respondents (33.3\%) rated the seminar slightly successful while two respondents (33.3\%) rated the seminar moderately successful.

The average rating of gardening without pain was $2.00(S D=.82)$ (see Table 22). The seminar on gardening without pain was rated unsuccessful bye one respondent (25.0\%). Two respondents (50.0\%) rated the seminar slightly successful. One respondent $(25.0 \%)$ rated the seminar moderately successful.

The average rating of drying flowers was $1.83(S D=.75)$ (see Table 22). Drying flowers was rated unsuccessful by two respondents (33.3\%). Three respondents (50.0\%) rated the seminar slightly successful while one respondent (16.7\%) rated it moderately successful.

The average rating of maintaining deck wood, maintaining outdoor furniture, preserving birdhouses and feeders and using landscape lighting to accent gardens at night was $1.67(S D=1.16)$. Two respondents $(66.7 \%)$ rated a seminar on maintaining deck wood unsuccessful in returning customers to the store. One respondent (33.3\%) rated the seminar moderately successful. Two respondents (66.7\%) rated maintaining outdoor furniture seminars unsuccessful in returning customers to the store. One respondent (33.3\%) rated the seminar moderately successful. Preserving birdhouses and feeders as a seminar was rated unsuccessful by two respondents (66.7\%). One respondent (33.3\%) rated the seminar moderately successful in returning customers to the store. Using landscape lighting to accent gardens at night was rated unsuccessful by two respondents (66.7\%). One respondent (33.3\%) rated a landscape lighting seminar as moderately successful in returning customers to the store (see Table 22). 
Table 22

Potential for Success in Getting Customers to Return to the Store-Educational Seminars

\begin{tabular}{|c|c|c|c|c|c|c|c|c|c|c|}
\hline & & & isucc & essful & $\begin{array}{l}\text { Sli } \\
\text { Suc }\end{array}$ & $\begin{array}{l}\text { htly } \\
\text { essful }\end{array}$ & Mod & $\begin{array}{l}\text { rately } \\
\text { essful }\end{array}$ & Suc & essful \\
\hline & $M$ & $S D$ & $N$ & $\%$ & $N$ & $\%$ & $N$ & $\%$ & $N$ & $\%$ \\
\hline Flower arranging & 3.00 & .58 & 0 & 0.0 & 1 & 14.3 & 5 & 71.4 & 1 & 14.3 \\
\hline Dividing perennials & 2.78 & .83 & 1 & 11.1 & 1 & 11.1 & 6 & 66.7 & 1 & 11.1 \\
\hline Cooking with herbs & 2.60 & .84 & 1 & 10.0 & 3 & 30.0 & 5 & 50.0 & 1 & 10.0 \\
\hline Herbal health remedies & 2.60 & 1.15 & 1 & 20.0 & 1 & 20.0 & 2 & 40.0 & 1 & 20.0 \\
\hline Plants that attract butterflies & 2.55 & 1.04 & 3 & 27.3 & 0 & 0.0 & 7 & 63.6 & 1 & 9.1 \\
\hline Pruning & 2.55 & .82 & 1 & 9.1 & 4 & 36.3 & 5 & 45.5 & 1 & 9.1 \\
\hline Gardening basics & 2.54 & .88 & 2 & 15.4 & 3 & 23.1 & 7 & 53.8 & 1 & 7.7 \\
\hline Planting & 2.54 & .78 & 12 & 40.0 & 18 & 60.0 & 0 & 0.0 & 0 & 0.0 \\
\hline Drying and storing herbs & 2.50 & 1.05 & 1 & 16.7 & 2 & 33.3 & 2 & 33.3 & 1 & 16.7 \\
\hline Preparing gardens for winter & 2.50 & 1.20 & 2 & 25.0 & 2 & 25.0 & 2 & 25.0 & 2 & 25.0 \\
\hline Plants that attract birds & 2.45 & 1.04 & 3 & 27.3 & 1 & 9.1 & 6 & 54.5 & 1 & 9.1 \\
\hline $\begin{array}{l}\text { Plants that repel pests (e. g. } \\
\text { deer, rabbits, etc) }\end{array}$ & 2.43 & 1.27 & 2 & 28.6 & 2 & 28.6 & 1 & 14.2 & 2 & 28.6 \\
\hline Identifying insect pests & 1.80 & .84 & 2 & 40.0 & 2 & 40.0 & 1 & 20.0 & 0 & 0.0 \\
\hline Dos and don'ts of mulch & 2.38 & 1.06 & 2 & 25.0 & 2 & 25.0 & 3 & 37.5 & 1 & 12.5 \\
\hline Identifying diseases & 2.00 & .89 & 2 & 33.3 & 2 & 33.3 & 2 & 33.3 & 0 & 0.0 \\
\hline Gardening without pain & 2.00 & .82 & 1 & 25.0 & 2 & 50.0 & 1 & 25.0 & 0 & 0.0 \\
\hline Drying flowers & 1.83 & .75 & 2 & 33.3 & 3 & 50.0 & 1 & 16.7 & 0 & 0.0 \\
\hline Maintaining deck wood & 1.67 & 1.16 & 2 & 66.7 & 0 & 0.0 & 1 & 33.3 & 0 & 0.0 \\
\hline Maintaining outdoor furniture & 1.67 & 1.16 & 2 & 66.7 & 0 & 0.0 & 1 & 33.3 & 0 & 0.0 \\
\hline $\begin{array}{l}\text { Preserving birdhouses and } \\
\text { feeders }\end{array}$ & 1.67 & 1.16 & 2 & 66.7 & 0 & 0.0 & 1 & 33.3 & 0 & 0.0 \\
\hline $\begin{array}{l}\text { Using landscape lighting to } \\
\text { accent gardens at night }\end{array}$ & 1.67 & 1.16 & 2 & 66.7 & 0 & 0.0 & 1 & 33.3 & 0 & 0.0 \\
\hline
\end{tabular}


Services for the Community. Using a scale of 1-4 with 1 being "unsuccessful" and 4 "successful" the average rating of donating plants to public areas was $2.33(S D=$ .73) (see Table 23). Donating plants to public areas was rated unsuccessful by two respondents $(9.5 \%)$ in returning customers to the store. Eleven respondents $(52.4 \%)$ rated donating plants slightly successful while seven respondents (33.2\%) rated donating plants moderately successful. One respondent (4.8\%) rated the service successful.

The average rating of donating supplies (e. g. mulch) was $2.30(S D=.80)$ (see Table 23). Twenty-one respondents (65.6\%) rated donating supplies (e. g. mulch) to public areas unsuccessful in returning customers to the store. Eleven respondents (34.4\%) rated donating supplies as slightly successful.

The average rating of local craft show was $2.14(S D=1.35)$ (see Table 23). Local craft shows were rated unsuccessful by three respondents (42.8\%) in returning customers to the store. Two respondents (28.6\%) rated the service slightly successful while two respondents $(28.6 \%)$ rated local craft shows successful.

The average rating of farmers market with free space for vendors was $1.71(S D=$ 1.25) (see Table 23). Farmers markets with free space for vendors were rated unsuccessful bye five respondents (71.4\%). Farmers markets with free space for vendors were rated moderately successful by one respondent (14.3\%) and successful bye one respondent (14.3\%). Farmers markets with paid space for vendors were rated unsuccessful by five respondents $(100.0 \%)(M=1.00, S D=.00)($ see Table 23$)$. 
Table 23

Potential for Success in Getting Customers to Return to the Store-Services for the Community

\begin{tabular}{|c|c|c|c|c|c|c|c|c|c|c|}
\hline & \multicolumn{4}{|c|}{ Unsuccessful } & \multicolumn{2}{|c|}{$\begin{array}{l}\text { Slightly } \\
\text { Successful }\end{array}$} & \multicolumn{2}{|c|}{$\begin{array}{l}\text { Moderately } \\
\text { Successful }\end{array}$} & \multicolumn{2}{|c|}{ Successful } \\
\hline & $M$ & $S D$ & $N$ & $\%$ & $N$ & $\%$ & $N$ & $\%$ & $N$ & $\%$ \\
\hline $\begin{array}{l}\text { Donation of plants to } \\
\text { public areas }\end{array}$ & 2.33 & .73 & 2 & 9.5 & 11 & 52.4 & 7 & 33.2 & 1 & 4.8 \\
\hline $\begin{array}{l}\text { Donation of supplies to } \\
\text { public areas }\end{array}$ & & & & & & & & & & \\
\hline (e. g. mulch) & 2.30 & .80 & 21 & 65.6 & 11 & 34.4 & 0 & 0.0 & 0 & 0.0 \\
\hline Local craft show & 2.14 & 1.35 & 3 & 42.8 & 2 & 28.6 & 0 & 0.0 & 2 & 28.6 \\
\hline $\begin{array}{l}\text { Farmers market with } \\
\text { free space for vendors }\end{array}$ & 1.71 & 1.25 & 5 & 71.4 & 0 & 0.0 & 1 & 14.3 & 1 & 14.3 \\
\hline $\begin{array}{l}\text { Farmers market with } \\
\text { paid space for vendors }\end{array}$ & 1.00 & .00 & 5 & 100.0 & 0 & 0.0 & 0 & 0.0 & 0 & 0.0 \\
\hline
\end{tabular}

Services for Customers. Using a scale of 1-4 with 1 being "unsuccessful" and 4 "successful" the average rating of customer designed containers (greenhouse grows until pickup date) was $3.47(S D=.62)$ (see Table 24). Customer designed containers grown by the greenhouse until picked up by the customer was rated slightly successful by one respondent (5.9\%). Seven respondents $(41.2 \%)$ rated the service moderately successful while nine respondents $(52.9 \%)$ rated the service successful in returning customers to the store.

The average rating of custom container design was $3.39(S D=.72)$ (see Table 24). Custom container design (e. g. hanging baskets, patio planters) was rated slightly 
successful by three respondents $(13.0 \%)$. Eight respondents $(34.8 \%)$ rated custom container design moderately successful while twelve respondents $(52.2 \%)$ rated custom container design successful in returning customers to the store.

The average rating of landscape installation was $3.25(S D=.97)$ (see Table 24). Landscape installation was rated unsuccessful bye two respondents $(10.0 \%)$. One respondent $(5.0 \%)$ rated the service slightly successful while seven respondents $(35.0 \%)$ rated landscape installation moderately successful. Ten respondents $(50.0 \%)$ rated landscape installation successful in returning customers to the store.

The average rating of landscape design was $3.19(S D=.93)$ (see Table 24). Landscape design services were rated unsuccessful by one respondent (4.8\%) in returning customers to the store. Four respondents (19.0\%) rated landscape design services slightly successful while six respondents $(28.6 \%)$ rated the service moderately successful. Ten respondents $(47.6 \%)$ rated landscape design services as successful in returning customers to the store.

The average rating of pruning shrubs was $3.14(S D=1.10)$ (see Table 24). Pruning shrubs was rated unsuccessful by two respondents $(14.3 \%)$. One respondent (7.1\%) rated pruning shrubs slightly successful while four respondents $(28.6 \%)$ rated the service moderately successful. Seven respondents $(50.0 \%)$ rated pruning shrubs successful in returning customers to the store.

The average rating of pruning trees was $2.90(S D=1.37)$ (see Table 24). Pruning trees was rated unsuccessful by three respondents (30.0\%). Two respondents $(20.0 \%)$ rated pruning trees moderately successful while five respondents $(50.0 \%)$ successful in returning customers to the store. 
The average rating of water garden design was $2.46(S D=1.13)$ (see Table 24). Water garden design was rated unsuccessful bye three respondents $(23.1 \%)$. Four respondents $(30.7 \%)$ rated water garden design as slightly successful while three respondents $(23.1 \%)$ rated it moderately successful. Three respondents $(23.1 \%)$ rated water garden design successful in returning customers to the store.

The average rating of water garden installation was $2.33(S D=1.23)$ (see Table 24). Water garden installation was rated unsuccessful by three respondents $(33.4 \%)$. Two respondents $(22.2 \%)$ rated water garden installation as slightly successful while two respondents $(22.2 \%)$ rated water garden installation moderately successful. Two respondents $(22.2 \%)$ rated water garden installation successful in returning customers to the garden center.

The average rating of rental plants for weddings was $2.18(S D=1.08)$ (see Table 24). Four respondents $(36.4 \%)$ rated rental plants for weddings unsuccessful in returning customers to the store. Two respondents $(18.2 \%)$ rated rental plants for weddings slightly successful while four respondents $(36.4 \%)$ rated the service moderately successful. One respondent $(9.0 \%)$ rated the service successful in returning customers to the store.

The average rating of floral design for weddings (e. g. corsages) was $2.13(S D=$ 1.36) (see Table 24). Floral design for weddings (e. g. corsages) was rated unsuccessful by four respondents $(50.0 \%)$. One respondent $(12.5 \%)$ rated floral design for weddings slightly successful while one respondent $(12.5 \%)$ rated the service moderately successful. Two respondents $(25.0 \%)$ rated floral design for weddings successful in returning customers to the store. 
The average rating of lawn equipment repair was $1.60(S D=1.34)$ (see Table 24). Lawn equipment repair was rated unsuccessful in returning customers to the store by two respondents (6.2\%). Thirty respondents (93.8\%) rated lawn equipment repair slightly successful (see Table 28).

The average rating of rental plants for offices was $1.50(S D=1.00)$ (see Table 24). Rental plants for offices were rated unsuccessful by three respondents $(75.0 \%)$. One respondent $(25.0 \%)$ rated rental plants for offices moderately successful in returning customers to the store.

Lines of Plant Material. Using a scale of 1-4 with 1 being "unsuccessful" and 4 "successful" the average rating of larger sizes of annuals was $3.67(S D=.48)$ (see Table 25). Larger sizes of annuals were rated moderately successful by seven respondents (33.3\%) while 14 respondents $(66.7 \%)$ rated larger sizes of annuals successful in returning customers to the store.

The average rating of summer annuals was $3.64(S D=.57)($ see Table 25). Summer annuals (e. g. wave petunias) were rated slightly successful by one respondent $(4.0 \%)$ in returning customers to the store. Seven respondents $(28.0 \%)$ rated summer annuals moderately successful. Seventeen respondents (68.0\%) rated summer annuals successful in returning customers to the store. 
Table 24

Potential for Success in Getting Customers to Return to the Store-Services for

Customers

Unsuccessful Slightly Moderately Successful

Successful Successful

$\begin{array}{llllllllll}M & S D & N & \% & N & \% & N & \% & N & \%\end{array}$

Customer designed containers

- greenhouse grows until

pickup date

$\begin{array}{llllllllll}3.47 & .62 & 0 & 0.0 & 1 & 5.9 & 7 & 41.2 & 9 & 52.9\end{array}$

Custom container design

(e. g. hanging baskets, patio

planters)

$\begin{array}{llllllllll}3.39 & .72 & 0 & 0.0 & 3 & 13.0 & 8 & 34.8 & 12 & 52.2\end{array}$

Landscape installation

$\begin{array}{llllllllll}3.25 & .97 & 2 & 10.0 & 1 & 5.0 & 7 & 35.0 & 10 & 50.0\end{array}$

Landscape design

$\begin{array}{llllllllll}3.19 & .93 & 1 & 4.8 & 4 & 19.0 & 6 & 28.6 & 10 & 47.6\end{array}$

Pruning shrubs

$\begin{array}{llllllllll}3.14 & 1.10 & 2 & 14.3 & 1 & 7.1 & 4 & 28.6 & 7 & 50.0\end{array}$

Pruning trees

$\begin{array}{llllllllll}2.90 & 1.37 & 3 & 30.0 & 0 & 0.0 & 2 & 20.0 & 5 & 50.0\end{array}$

Water garden design

$\begin{array}{llllllllll}2.46 & 1.13 & 3 & 23.1 & 4 & 30.7 & 3 & 23.1 & 3 & 23.1\end{array}$

Water garden installation

$\begin{array}{llllllllll}2.33 & 1.23 & 3 & 33.4 & 2 & 22.2 & 2 & 22.2 & 2 & 22.2\end{array}$

Rental plants for weddings

$\begin{array}{llllllllll}2.18 & 1.08 & 4 & 36.4 & 2 & 18.2 & 4 & 36.4 & 1 & 9.0\end{array}$

Floral design for weddings (e.

g. corsages)

$\begin{array}{llllllllll}2.13 & 1.36 & 4 & 50.0 & 1 & 12.5 & 1 & 12.5 & 2 & 25.0\end{array}$

Lawn equipment repair

$\begin{array}{llllllllll}1.60 & 1.34 & 2 & 6.2 & 30 & 93.8 & 0 & 0.0 & 0 & 0.0\end{array}$

Rental plants for offices

$\begin{array}{llllllllll}1.50 & 1.00 & 3 & 75.0 & 0 & 0.0 & 1 & 25.0 & 0 & 0.0\end{array}$ 
The average rating of perennials was $3.52(S D=.58)$ (see Table 25). Perennials were rated slightly successful by one respondent (3.7\%) in returning customers to the store. Eleven respondents $(40.7 \%)$ rated perennials moderately successful while 15 respondents $(55.6 \%)$ rated perennials successful in returning customers to the store.

The average rating of larger sizes of perennials was $3.42(S D=.65)$ (see Table 25). Larger sizes of perennials were rated slightly successful in returning customers to the store by two respondents $(8.3 \%)$. Ten respondents $(41.7 \%)$ rated larger sizes of perennials moderately successful while 12 respondents $(50.0 \%)$ rated larger sizes of perennials successful in returning customers to the store.

The average rating of summer blooming perennials was $3.41(S D=.69)$ (see Table 25). Summer blooming perennials were rated slightly successful by three respondents $(11.1 \%)$. Ten respondents $(37.0 \%)$ rated summer blooming perennials moderately successful in returning customers to the store while 14 respondents $(51.9 \%)$ rated summer blooming perennials successful.

The average rating of outdoor container gardens was $3.36(S D=.72)$ (see Table 25). Outdoor container gardens were rated slightly successful by three respondents $(13.6 \%)$. Eight respondents $(36.4 \%)$ rated outdoor container gardens moderately successful in returning customers to the store and 11 respondents $(50.0 \%)$ rated outdoor container gardens successful in returning customers to the store.

The average rating of cool weather annuals (e. g. pansies) was $3.35(S D=.71)$ (see Table 25). Cool weather annuals (e. g. pansies) were rated slightly successful by three respondents $(13.1 \%)$. Nine respondents $(39.1 \%)$ rated cool weather annuals 
moderately successful while 11 respondents $(47.8 \%)$ rated the plant material successful in returning customers to the store.

The average rating of fall blooming ornamental grasses was $3.17(S D=.70)$ (see Table 25). Fall blooming ornamental grasses were rated slightly successful by four respondents (16.7\%). Twelve respondents (50.0\%) rated fall blooming ornamental grasses moderately successful while 8 respondents $(33.3 \%)$ rated the plant material successful in returning customers to the store.

The average rating of windowsill herb pots was $3.06(S D=.80)($ see Table 25$)$. Windowsill herb pots were rated slightly successful by five respondents $(27.8 \%)$. Seven respondents $(38.9 \%)$ rated windowsill herb pots moderately successful in returning customers to the store while six respondents (33.3\%) rated windowsill herb pots successful.

The average rating of plant propagation supplies (e. g. seed potatoes, onion sets and garden seeds) was $2.94(S D=.97)$ (see Table 25). Plant propagation supplies (e. g. seed potatoes, onion sets and garden seeds) were rated unsuccessful in returning customers to the store by 17 respondents $(53.1 \%)$. Fifteen respondents $(46.9 \%)$ rated plant propagation supplies slightly successful in returning customers to the stor.

The average rating of herb topiaries was $2.82(S D=.75)$ (see Table 25). Four respondents (36.4\%) rated herb topiaries slightly successful in returning customers to the store. Five respondents (45.4\%) rated herb topiaries moderately successful while two respondents (18.2\%) rated herb topiaries successful in returning customers to the store.

The average rating of house plants (e. g. African violets, ivy, etc) was $2.78(S D=$ 1.06) (see Table 25). House plants (e. g. African violets, ivy, etc) were rated 
unsuccessful by three respondents $(16.7 \%)$. Three respondents $(16.7 \%)$ rated house plants slightly successful in returning customers to the store while seven respondents (38.8\%) rated house plants moderately successful. Five respondents $(27.8 \%)$ rated house plants successful.

The average rating of orchids was $2.69(S D=1.18)$ (see Table 25). Orchids were rated unsuccessful in returning customers to the store by three respondents (23.1\%). Two respondents (15.3\%) rated orchids slightly successful while four respondents (30.8\%) rated orchids moderately successful. Four respondents (30.8\%) rated orchids successful in returning customers to the store.

The average rating of container evergreens for indoors was $2.67(S D=1.23)$ (see Table 25). Container evergreens for indoors were rated unsuccessful by two respondents (22.2\%). Two respondents (22.2\%) rated container evergreens for indoors slightly successful in returning customers to the store while two respondents $(22.2 \%)$ rated them moderately successful. Three respondents (33.4\%) rated container evergreens for indoors successful in returning customers to the store.

The average rating of fall bulbs (e. g. tulips) was $2.62(S D=1.15)($ see Table 25). Fall bulbs (e. g. tulips) were rated unsuccessful by three respondents (18.8\%). Five respondents (31.2\%) rated fall bulbs slightly successful in returning customers to the store while three respondents (18.8\%) rated fall bulbs moderately successful. Five respondents (31.2\%) rated fall bulbs successful in returning customers to the store.

The average rating of fresh cut flowers year round was $2.57(S D=1.27)$ (see Table 25). Two respondents (28.6\%) rated fresh cut flowers year round unsuccessful in returning customers to the store. One respondent (14.3\%) rated fresh cut flowers year 
round slightly successful while two respondents (28.6\%) rated fresh cut flowers year round moderately successful. Two respondents (28.6\%) rated fresh cut flowers year round successful in returning customers to the store.

The average rating of tropical bulbs (e. g. caladium) was $2.43(S D=1.01)$ (see Table 25). Tropical bulbs (e. g. caladium) were rated unsuccessful by three respondents (21.4\%). Four respondents (28.6\%) rated tropical bulbs slightly successful while five respondents (35.7\%) rated tropical bulbs moderately successful in returning customers to the store. Two respondents (14.3\%) rated tropical bulbs successful.

The average rating of spring bulbs (e. g. dahlias) was $2.14(S D=1.02)$ (see Table 25). Four respondents (28.5\%) rated spring bulbs (e. g. dahlias) unsuccessful in returning customers to the store. Six respondents (42.9\%) rated spring bulbs slightly successful while two respondents $(14.3 \%)$ rated spring bulbs moderately successful. Two respondents (14.3\%) rated spring bulbs successful in returning customers to the store. 
Table 25

Potential for Success in Getting Customers to Return to the Store-Lines of Plant Material

Unsuccessful Slightly ModeratelySuccessful Successful Successful

\begin{tabular}{llllllllll}
\hline$M$ & $S D$ & $N$ & $\%$ & $N$ & $\%$ & $N$ & $\%$ & $N$ & $\%$
\end{tabular}

Larger sizes of annuals

$\begin{array}{llllllllll}3.67 & .48 & 0 & 0.0 & 0 & 0.0 & 7 & 33.3 & 14 & 66.7\end{array}$

Summer annuals (e. g. wave petunias)

$\begin{array}{llllllllll}3.64 & .57 & 0 & 0.0 & 1 & 4.0 & 7 & 28.0 & 17 & 68.0\end{array}$

Perennials

$\begin{array}{llllllllll}3.52 & .58 & 0 & 0.0 & 1 & 3.7 & 11 & 40.7 & 15 & 55.6\end{array}$

Larger sizes of perennials

$\begin{array}{llllllllll}3.42 & .65 & 0 & 0.0 & 2 & 8.3 & 10 & 41.7 & 12 & 50.0\end{array}$

Summer blooming perennials

$\begin{array}{llllllllll}3.41 & .69 & 0 & 0.0 & 3 & 11.1 & 10 & 37.0 & 14 & 51.9\end{array}$

Outdoor container gardens

$\begin{array}{llllllllll}3.36 & .72 & 0 & 0.0 & 3 & 13.6 & 8 & 36.4 & 11 & 50.0\end{array}$

Cool weather annuals (e. g. pansies)

$\begin{array}{llllllllll}3.35 & .71 & 0 & 0.0 & 3 & 13.1 & 9 & 39.1 & 11 & 47.8\end{array}$

Fall blooming ornamental grasses

$\begin{array}{llllllllll}3.17 & .70 & 0 & 0.0 & 4 & 16.7 & 12 & 50.0 & 8 & 33.3\end{array}$

Windowsill herb pots

$\begin{array}{llllllllll}3.06 & .80 & 0 & 0.0 & 5 & 27.8 & 7 & 38.9 & 6 & 33.3\end{array}$

Plant propagation supplies (e. g. seed potatoes, onion sets and garden seeds)

$\begin{array}{llllllllll}2.94 & .97 & 17 & 53.1 & 15 & 46.9 & 0 & 0.0 & 0 & 0.0\end{array}$

Herb topiaries

$\begin{array}{llllllllll}2.82 & .75 & 0 & 0.0 & 4 & 36.4 & 5 & 45.4 & 2 & 18.2\end{array}$

House plants (e. g. African

violets, ivy, etc)

$\begin{array}{llllllllll}2.78 & 1.06 & 3 & 16.7 & 3 & 16.7 & 7 & 38.8 & 5 & 27.8\end{array}$

Orchids

$\begin{array}{llllllllll}2.69 & 1.18 & 3 & 23.1 & 2 & 15.3 & 4 & 30.8 & 4 & 30.8\end{array}$

Container evergreens for indoors $\begin{array}{llllllllll}2.67 & 1.23 & 2 & 22.2 & 2 & 22.2 & 2 & 22.2 & 3 & 33.4\end{array}$

$\begin{array}{lllllllllll}\text { Fall bulbs (e. g. tulips) } & 2.62 & 1.15 & 3 & 18.8 & 5 & 31.2 & 3 & 18.8 & 5 & 31.2\end{array}$

$\begin{array}{lllllllllll}\text { Fresh cut flowers year round } & 2.57 & 1.27 & 2 & 28.6 & 1 & 14.3 & 2 & 28.6 & 2 & 28.6\end{array}$

$\begin{array}{lllllllllll}\text { Tropical bulbs (e. g. caladium) } & 2.43 & 1.01 & 3 & 21.4 & 4 & 28.6 & 5 & 35.7 & 2 & 14.3\end{array}$

$\begin{array}{lllllllllll}\text { Spring bulbs (e. g. dahlias) } & 2.14 & 1.02 & 4 & 28.5 & 6 & 42.9 & 2 & 14.3 & 2 & 14.3\end{array}$ 
Lines of Merchandise. Using a scale of 1-4 with 1 being "unsuccessful" and 4 "successful" the average rating of hard goods was $3.38(S D=.74)$ (see Table 26). Hard goods (e. g. pottery, statues, etc) were rated slightly successful by three respondents (14.3\%). Seven respondents (33.3\%) rated hard goods moderately successful while 11 respondents (52.4\%) rated hard goods successful in returning customers to the store.

The average rating of wind chimes was $3.00(S D=1.16)$ (see Table 26). Wind chimes were rated unsuccessful by two respondents (12.5\%). Four respondents $(25.0 \%)$ rated wind chimes slightly successful in returning customers to the store while two respondents (12.5\%) rated wind chimes moderately successful. Eight respondents (50.0\%) rated wind chimes successful in returning customers to the store.

The average rating of hummingbird supplies was $2.71(S D=1.05)$ (see Table 26). Hummingbird supplies were rated unsuccessful by two respondents (11.8\%). Six respondents $(35.3 \%)$ rated hummingbird supplies slightly successful in returning customers to the store while four respondents (23.5\%) rated hummingbird supplies moderately successful. Five respondents (29.4\%) rated hummingbird supplies successful in returning customers to the store.

The average rating of garden flags was $2.64(S D=1.22)$ (see Table 26). Garden flags were rated unsuccessful by three respondents (21.4\%). Four respondents $(28.6 \%)$ rated garden flags slightly successful in returning customers to the store while two respondents (14.3\%) rated garden flags moderately successful. Five respondents (35.7\%) rated garden flags successful.

The average rating of outdoor furniture and accessories was $2.53(S D=1.18)$ (see Table 26). Outdoor furniture and accessories were rated unsuccessful by four 
respondents $(26.7 \%)$. Three respondents (19.9\%) rated outdoor furniture and accessories slightly successful while four respondents $(26.7 \%)$ rated the merchandise moderately successful. Four respondents (26.7\%) rated outdoor furniture and accessories successful in returning customers to the store.

The average rating of candles and accessories was $2.36(S D=.93)$ (see Table 26). Candles and accessories were rated unsuccessful by two respondents (14.3\%). Seven respondents $(50.0 \%)$ rated candles and accessories slightly successful while three respondents $(21.4 \%)$ rated candles and accessories moderately successful. Two respondents $(14.3 \%)$ rated candles and accessories successful in returning customers to the store.

The average rating of wildlife/bird feeding supplies was $2.25(S D=1.34)$ (see Table 26). Wildlife/bird feeding supplies were rated unsuccessful in returning customers to the store by seven respondents $(43.6 \%)$. Three respondents $(18.8 \%)$ rated the merchandise slightly successful. One respondent (6.3\%) rated wildlife/bird feeding supplies moderately successful while five respondents $(31.3 \%)$ rated the merchandise successful in returning customers to the store.

The average rating of pet food and supplies was $2.20(S D=1.64)$ (see Table 26). Pet food and supplies were rated unsuccessful in returning customers to the store by three respondents $(60.0 \%)$. Two respondents $(40.0 \%)$ rated pet food and supplies successful.

The average rating of lawn equipment (e. g. mowers, weed trimmers) was 2.00 $(S D=1.41)($ see Table 26$)$. Lawn equipment was rated unsuccessful by three respondents $(60.0 \%)$. One respondent $(20.0 \%)$ rated lawn equipment moderately 
successful in returning customers to the store while one respondent $(20.0 \%)$ rated lawn equipment successful.

The average rating of local honey was $2.00(S D=1.41)$ (see Table 25). Two respondents $(50.0 \%)$ rated local honey unsuccessful in returning customers to the store. One respondent $(25.0 \%)$ rated local honey slightly successful while one respondent $(25.0 \%)$ rated it successful.

The average rating of garden themed clothing was $1.80(S D=1.30)$ (see Table 26). Garden themed clothing was rated unsuccessful by three respondents $(60.0 \%)$. One respondent $(20.0 \%)$ rated garden themed clothing slightly successful while one respondent $(20.0 \%)$ rated the merchandise successful in returning customers to the store.

The average rating of local jams and jellies was $1.33(S D=.58)$ (see Table 26). Local jams and jellies were rated unsuccessful by two respondents $(66.7 \%)$ while one respondent (33.3\%) rated local jams and jellies slightly successful.

The average rating of local wine, other wine, canning supplies and other honey was $1.25(S D=.50)$ (see Table 26$)$. Local wine was rated unsuccessful in returning customers to the store by two respondents $(66.7 \%)$. One respondent $(33.3 \%)$ rated local wine slightly successful. Other wine was rated unsuccessful by three respondents $(75.0 \%)$ while one respondent $(25.0 \%)$ rated other wine slightly successful. Canning supplies were rated unsuccessful in returning customers to the store by three respondents $(75.0 \%)$. One respondent $(25.0 \%)$ rated canning supplies slightly successful. Other honey was rated unsuccessful by three respondents $(75.0 \%)$ while one respondent rated it slightly successful $(25.0 \%)$. 
The average rating of other jams and jellies was $1.00(S D=.00)$ (see Table 26). One respondent (3.3\%) rated other jams and jellies unsuccessful in returning customers to the store while 29 respondents $(96.7 \%)$ rated other jams and jellies slightly successful (see Table 30).

Adjustments to Business or Location. Using a scale of 1-4 with 1 being "unsuccessful" and 4 "successful" the average rating of customer friendly staff was 3.89 $(S D=.32)($ see Table 27$)$. Three respondents $(11.1 \%)$ rated customer friendly staff moderately successful. Twenty four respondents $(88.9 \%)$ rated customer friendly staff successful in returning customers to the store.

The average rating of knowledgeable and experienced staff was $3.85(S D=.46)$ (see Table 27). Knowledgeable and experienced staff was rated slightly successful by one respondent (3.7\%). Two respondents (7.4\%) rated knowledgeable and experienced staff moderately successful in returning customers to the store while 24 respondents $(88.9 \%)$ rated the adjustment successful.

The average rating of growing your own plants to allow for diverse product mix was $3.74(S D=.45)$ (see Table 27). Growing your own plants to allow for diverse product mix was rated moderately successful by five respondents $(26.3 \%)$. Fourteen respondents $(73.7 \%)$ rated growing your own plants to allow for diverse product mix successful in returning customers to the store. 
Table 26

Potential for Success in Getting Customers to Return to the Store-Lines of Merchandise Unsuccessful Slightly Moderately Successful Successful Successful

$\begin{array}{llllllllll}M & S D & N & \% & N & \% & N & \% & N & \%\end{array}$

Hard goods (e. g. pottery, statues, etc)

$\begin{array}{llllllllll}3.38 & .74 & 0 & 0.0 & 3 & 14.3 & 7 & 33.3 & 11 & 52.4\end{array}$

Wind chimes

$\begin{array}{llllllllll}3.00 & 1.16 & 2 & 12.5 & 4 & 25.0 & 2 & 12.5 & 8 & 50.0\end{array}$

Hummingbird supplies

$\begin{array}{llllllllll}2.71 & 1.05 & 2 & 11.8 & 6 & 35.3 & 4 & 23.5 & 5 & 29.4\end{array}$

Garden flags

$\begin{array}{llllllllll}2.64 & 1.22 & 3 & 21.4 & 4 & 28.6 & 2 & 14.3 & 5 & 35.7\end{array}$

Outdoor furniture and

accessories

$\begin{array}{llllllllll}2.53 & 1.18 & 4 & 26.7 & 3 & 19.9 & 4 & 26.7 & 4 & 26.7\end{array}$

Candles and accessories

$\begin{array}{llllllllll}2.36 & .93 & 2 & 14.3 & 7 & 50.0 & 3 & 21.4 & 2 & 14.3\end{array}$

$\begin{array}{lllllllllll}\text { Wildlife/bird feeding supplies } & 2.25 & 1.34 & 7 & 43.6 & 3 & 18.8 & 1 & 6.3 & 5 & 31.3\end{array}$

Pet food and supplies

$\begin{array}{llllllllll}2.20 & 1.64 & 3 & 60.0 & 0 & 0.0 & 0 & 0.0 & 2 & 40.0\end{array}$

Lawn equipment (e. g.

mowers, weed trimmers)

$\begin{array}{llllllllll}2.00 & 1.41 & 3 & 60.0 & 0 & 0.0 & 1 & 20.0 & 1 & 20.0\end{array}$

Local honey

$\begin{array}{llllllllll}2.00 & 1.41 & 2 & 50.0 & 1 & 25.0 & 0 & 0.0 & 1 & 25.0\end{array}$

Garden themed clothing

$\begin{array}{llllllllll}1.80 & 1.30 & 3 & 60.0 & 1 & 20.0 & 0 & 0.0 & 1 & 20.0\end{array}$

Local jams and jellies

$\begin{array}{llllllllll}1.33 & .58 & 2 & 66.7 & 1 & 33.3 & 0 & 0.0 & 0 & 0.0\end{array}$

Local wine

$\begin{array}{llllllllll}1.33 & .58 & 2 & 66.7 & 1 & 33.3 & 0 & 0.0 & 0 & 0.0\end{array}$

Other wine

$\begin{array}{llllllllll}1.25 & .50 & 3 & 75.0 & 1 & 25.0 & 0 & 0.0 & 0 & 0.0\end{array}$

Canning supplies

$\begin{array}{llllllllll}1.25 & .50 & 3 & 75.0 & 1 & 25.0 & 0 & 0.0 & 0 & 0.0\end{array}$

Other honey

$\begin{array}{llllllllll}1.25 & .50 & 3 & 75.0 & 1 & 25.0 & 0 & 0.0 & 0 & 0.0\end{array}$

Other jams and jellies

$\begin{array}{llllllllll}1.00 & .00 & 1 & 3.3 & 29 & 96.7 & 0 & 0.0 & 0 & 0.0\end{array}$ 
The average rating of neat and clean aisles and displays was $3.60(S D=.65)($ see Table 27). Neat and clean aisles and displays were rated slightly successful by two respondents $(8.0 \%)$. Six respondents $(24.0 \%)$ rated neat and clean aisles and displays moderately successful in returning customers to the store while 17 respondents $(68.0 \%)$ rated neat and clean aisles and displays successful.

The average rating of display gardens was $3.50(S D=.63)$ (see Table 27). One respondent $(6.3 \%)$ rated display gardens slightly successful in returning customers to the store. Six respondents (37.4\%) rated display gardens moderately successful while nine respondents $(56.3 \%)$ rated display gardens successful in returning customers to the store.

The average rating of establishing a "greeter" at the entrance was $3.46(S D=.66)$ (see Table 27). Establishing a "greeter" at the entrance was rated slightly successful in returning customers to the store by one respondent (7.7\%). Five respondents $(38.5 \%)$ rated the adjustment moderately successful while seven respondents $(53.8 \%)$ rated establishing a "greeter" at the entrance successful.

The average rating of raise prices on all items was $3.31(S D=.95)$ (see Table 27). Raising prices on all items was rated unsuccessful by one respondent (6.3\%). Two respondents $(12.5 \%)$ rated raising prices on all items slightly successful while four respondents $(25.0 \%)$ rated it moderately successful. Nine respondents $(56.2 \%)$ rated raising prices on all items successful in returning customers to the store.

The average rating of establish a warranty for plant material was $3.20(S D=.77)$ (see Table 27 ). One respondent (5.0\%) rated establishing a warranty for plant material unsuccessful in returning customers to the store. One respondent rated the adjustment 
slightly successful while 11 respondents (55.0\%) rated it moderately successful. Seven respondents (35.0\%) rated establishing a warranty for plant material successful.

The average rating of add a covered shopping area for year round sales was 3.14 $(S D=1.10)$ (see Table 27). Two respondents $(14.3 \%)$ rated adding a covered shopping area for year round sales unsuccessful in returning customers to the store. One respondent (7.1\%) rated it slightly successful while four respondents $(28.6 \%)$ rated the adjustment moderately successful. Seven respondents (50.0\%) rated adding a covered shopping area for year round sales successful.

The average rating of hiring a certified horticulturist was $3.09(S D=1.22)$. Two respondents (18.2\%) rated hiring a certified horticulturist unsuccessful. One respondent (9.1\%) rated hiring a certified horticulturist slightly successful while two respondents (18.2\%) rated it moderately successful. Six respondents (54.5\%) rated hiring a certified horticulturist successful in returning customers to the store.

The average rating of become bonded was $2.75(S D=1.28)$ (see Table 27). Seven respondents (24.1\%) rated becoming bonded unsuccessful in returning customers to the store. Twenty two respondents (75.9\%) rated becoming bonded slightly successful in returning customers to the store.

The average rating of convert greenhouse to banquet hall during non-peak months was $1.75(S D=.96)$ (see Table 27). Two respondents (50.0\%) rated converting the greenhouse to a banquet hall during non-peak months unsuccessful. One respondent (25.0\%) rated it slightly successful while one respondent (25.0\%) rated it moderately successful. 
The average rating of add a bakery was $1.67(S D=1.16)$ (see Table 27). Two respondents $(66.7 \%)$ rated adding a bakery unsuccessful in returning customers to the garden center. One respondent (33.3\%) rated adding a bakery moderately successful.

The average rating of add an ice cream shop was $1.67(S D=1.16)$. Adding an ice cream shop was rated unsuccessful in returning customers to the store by two respondents (66.7\%). One respondent (33.3\%) rated adding an ice cream shop moderately successful in returning customers to the store.

The average rating of add a coffee house was $1.60(S D=.89)$ (see Table 27). Three respondents $(60.0 \%)$ rated adding a coffee house unsuccessful in returning customers to the store. One respondent $(20.0 \%)$ rated adding a coffee house slightly successful while one respondent $(20.0 \%)$ rated it moderately successful. 
Table 27

Potential for Success in Getting Customers to Return to the Store-Adjustments to Business or Location

\begin{tabular}{|c|c|c|c|c|c|c|c|c|c|c|}
\hline & \multicolumn{4}{|c|}{ Unsuccessful } & \multicolumn{2}{|c|}{$\begin{array}{l}\text { Slightly } \\
\text { Successful }\end{array}$} & \multicolumn{4}{|c|}{$\begin{array}{l}\text { Moderately Successful } \\
\text { Successful }\end{array}$} \\
\hline & $M$ & $S D$ & $N$ & $\%$ & $N$ & $\%$ & $N$ & $\%$ & $N$ & $\%$ \\
\hline Customer friendly staff & 3.89 & .32 & 0 & 0.0 & 0 & 0.0 & 3 & 11.1 & 24 & 88.9 \\
\hline $\begin{array}{l}\text { Knowledgeable and } \\
\text { experienced staff }\end{array}$ & 3.85 & .46 & 0 & 0.0 & 1 & 3.7 & 2 & 7.4 & 24 & 88.9 \\
\hline $\begin{array}{l}\text { Growing your own plants to } \\
\text { allow for diverse product mix }\end{array}$ & 3.74 & .45 & 0 & 0.0 & 0 & 0.0 & 5 & 26.3 & 14 & 73.7 \\
\hline $\begin{array}{l}\text { Neat and clean aisles and } \\
\text { displays }\end{array}$ & 3.60 & .65 & 0 & 0.0 & 2 & 8.0 & 6 & 24.0 & 17 & 68.0 \\
\hline Display gardens & 3.50 & .63 & 0 & 0.0 & 1 & 6.3 & 6 & 37.4 & 9 & 56.3 \\
\hline $\begin{array}{l}\text { Establish a "greeter" at } \\
\text { entrance }\end{array}$ & 3.46 & .66 & 0 & 0.0 & 1 & 7.7 & 5 & 38.5 & 7 & 53.8 \\
\hline Raise prices on all items & 3.31 & .95 & 1 & 6.3 & 2 & 12.5 & 4 & 25.0 & 9 & 56.2 \\
\hline $\begin{array}{l}\text { Establish warranty for plant } \\
\text { material }\end{array}$ & 3.20 & .77 & 1 & 5.0 & 1 & 5.0 & 11 & 55.0 & 7 & 35.0 \\
\hline $\begin{array}{l}\text { Add a covered shopping area } \\
\text { for year round sales }\end{array}$ & 3.14 & 1.10 & 2 & 14.3 & 1 & 7.1 & 4 & 28.6 & 7 & 50.0 \\
\hline Hire a certified horticulturist & 3.09 & 1.22 & 2 & 18.2 & 1 & 9.1 & 2 & 18.2 & 6 & 54.5 \\
\hline Become bonded & 2.75 & 1.28 & 7 & 24.1 & 22 & 75.9 & 0 & 0.0 & 0 & 0.0 \\
\hline $\begin{array}{l}\text { Convert greenhouse to } \\
\text { banquet hall during non-peak } \\
\text { months }\end{array}$ & 1.75 & .96 & 2 & 50.0 & 1 & 25.0 & 1 & 25.0 & 0 & 0.0 \\
\hline Add a bakery & 1.67 & 1.16 & 2 & 66.7 & 0 & 0.0 & 1 & 33.3 & 0 & 0.0 \\
\hline Add an ice cream shop & 1.67 & 1.16 & 2 & 66.7 & 0 & 0.0 & 1 & 33.3 & 0 & 0.0 \\
\hline Add a coffee house & 1.60 & .89 & 3 & 60.0 & 1 & 20.0 & 1 & 20.0 & 0 & 0.0 \\
\hline
\end{tabular}


Customer Appreciation. Using a scale of 1-4 with 1 being "unsuccessful" and 4 "successful" the average rating of percent-off sale events was $3.20(S D=.70)$ (see Table 28). Percent-off sale events were rated slightly successful in returning customers to the store by three respondents $(15.0 \%)$. Ten respondents $(50.0 \%)$ rated percent-off sale events were rated moderately successful. Seven respondents (35.0\%) rated percent-off sale events successful in returning customers to the store.

The average rating of seasonally dated coupons was $3.18(S D=.73)$ (see Table 28). Seasonally dated coupons were rated slightly successful by three respondents (17.6\%). Eight respondents (47.1\%) rated seasonally dated coupons moderately successful in returning customers to the store while six respondents (35.3\%) rated seasonally dated coupons successful.

The average rating of holiday specials on corresponding plants and accessories (i.e. Memorial Day: red, white and blue) was $2.80(S D=1.14)$ (see Table 28). Holiday specials on corresponding plants and accessories were rated unsuccessful by two respondents $(20.0 \%)$. One respondent $(10.0 \%)$ rated the method of customer appreciation slightly successful while four respondents $(40.0 \%)$ rated it moderately successful. Three respondents (30.0\%) rated the customer appreciation method successful in returning customers to the store.

The average rating of spend $\$ 50$ get free gift was $2.78(S D=.67)$ (see Table 28). Three respondents (33.3\%) rated a spend $\$ 50$ get free gift event slightly successful while five respondents (55.6\%) rated the method of customer appreciation moderately successful. One respondent (11.1\%) rated the method successful in returning customers to the store. 
The average rating of offer extra stamps on loyalty cards in non-peak months was $2.71(S D=.76)$ (see Table 28$)$. Offering extra stamps on loyalty cards in non-peak months was rated unsuccessful by three respondents $(42.9 \%)$. Three respondents $(42.9 \%)$ rated the method of customer appreciation moderately successful. One respondent $(14.2 \%)$ rated the method successful in returning customers to the store.

The average rating of sell gift certificates at 20 percent off during non-peak months was $2.56(S D=.88)$ (see Table 28$)$. Selling gift certificates at 20 percent off during non-peak months was rated unsuccessful by one respondent (11.1\%). Three respondents (33.4\%) rated this method of customer appreciation slightly successful while four respondents $(44.4 \%)$ rated it moderately successful. One respondent $(11.1 \%)$ rated selling gift certificates at 20 percent off during non-peak months was rated successful.

The average rating of offer specials to customers in costume on Halloween was $1.67(S D=1.16)($ see Table 28). Offering specials to customers in costume on Halloween was rated unsuccessful by two respondents $(66.7 \%)$. One respondent $(33.3 \%)$ rated the customer appreciation method moderately successful. 
Table 28

Potential for Success in Getting Customers to Return to the Store-Customer Appreciation

\begin{tabular}{|c|c|c|c|c|c|c|c|c|c|c|}
\hline & \multicolumn{4}{|c|}{ Unsuccessful } & \multicolumn{2}{|c|}{$\begin{array}{l}\text { Slightly } \\
\text { Successful }\end{array}$} & \multicolumn{2}{|c|}{$\begin{array}{l}\text { Moderately } \\
\text { Successful }\end{array}$} & \multicolumn{2}{|c|}{ Successful } \\
\hline & $M$ & $S D$ & $N$ & $\%$ & $N$ & $\%$ & $N$ & $\%$ & $N$ & $\%$ \\
\hline Percent-off sale events & 3.20 & .70 & 0 & 0.0 & 3 & 15.0 & 10 & 50.0 & 7 & 35.0 \\
\hline Seasonally dated coupons & 3.18 & .73 & 0 & 0.0 & 3 & 17.6 & 8 & 47.1 & 6 & 35.3 \\
\hline $\begin{array}{l}\text { Holiday specials on } \\
\text { corresponding plants and } \\
\text { accessories i.e. Memorial } \\
\text { Day: red, white and blue }\end{array}$ & 2.80 & 1.14 & 2 & 20.0 & 1 & 10.0 & 4 & 40.0 & 3 & 30.0 \\
\hline Spend $\$ 50$ get __ free & 2.78 & .67 & 0 & 0.0 & 3 & 33.3 & 5 & 55.6 & 1 & 11.1 \\
\hline $\begin{array}{l}\text { Offer extra stamps on loyalty } \\
\text { cards in non-peak months }\end{array}$ & 2.71 & .76 & 0 & 0.0 & 3 & 42.9 & 3 & 42.9 & 1 & 14.2 \\
\hline $\begin{array}{l}\text { Sell gift certificates at } 20 \\
\text { percent off during non-peak } \\
\text { months }\end{array}$ & 2.56 & .88 & 1 & 11.1 & 3 & 33.4 & 4 & 44.4 & 1 & 11.1 \\
\hline $\begin{array}{l}\text { Offer specials to customers in } \\
\text { costume on Halloween }\end{array}$ & 1.67 & 1.16 & 2 & 66.7 & 0 & 0.0 & 1 & 33.3 & 0 & 0.0 \\
\hline
\end{tabular}

Potential for Success in Raising Profit Margins

Respondents were asked to rate the practices on their potential for success in raising profit margins. Respondents were to rate the practice on the following scale: (4) Successful, (3) Moderately Successful, (2) Slightly Successful, (1) Unsuccessful. 
Holiday Merchandise or Services. Using a scale of 1-4 with 1 being "unsuccessful" and 4 "successful" the average rating of custom made decorations (e. g. wreaths, swags) was $2.90(S D=1.04)$ (see Table 29). Custom made decorations (e. g. wreaths, swags) were rated unsuccessful by two respondents (9.5\%). Six respondents (28.6\%) rated custom made decorations slightly successful while five respondents $(23.8 \%)$ rated them moderately successful. Eight respondents (38.1\%) rated custom made decorations successful in raising profit margins.

The average rating of Christmas gift plants (e. g. poinsettias) was 2.85 ( $S D=$ 1.23) (see Table 29). Christmas gift plants were rated unsuccessful by four respondents (20.0\%). Four respondents (20.0\%) rated Christmas gift plants slightly successful while three respondents (15.0\%) rated the plants moderately successful. Nine respondents $(45.0 \%)$ rated Christmas gift plants successful in raising profit margins.

The average rating of painted poinsettias was $2.85(S D=1.28)($ see Table 29). Painted poinsettias were rated unsuccessful by three respondents (23.1\%) while two respondents (15.4\%) rated them slightly successful. Two respondents (15.4\%) rated painted poinsettias moderately successful and six respondents (46.2\%) rated painted poinsettias successful in raising profit margins.

The average rating of container trees was $2.85(S D=1.14)$ (see Table 29). Container trees were rated unsuccessful by three respondents (15.0\%). Five respondents $(25.0 \%)$ rated container trees slightly successful in raising profit margins while four respondents (20.0\%) rated them moderately successful. Eight respondents (40.0\%) rated container trees successful in raising profit margins. 
The average rating of balled and burlap trees was $2.65(S D=1.06)$ (see Table 29). Balled and burlap trees were rated unsuccessful by two respondents (11.8\%). Seven respondents (41.2\%) rated balled and burlap trees slightly successful while three respondents (17.6\%) rated balled and burlap trees moderately successful. Five respondents (29.4\%) rated balled and burlap trees successful in raising profit margins.

The average rating of live pumpkins was $2.62(S D=.81)$ (see Table 29). Live pumpkins were rated unsuccessful by one respondent (4.8\%). Nine respondents $(42.9 \%)$ rated live pumpkins slightly successful in raising profit margins while eight respondents (38.1\%) rated live pumpkins moderately successful. Three respondents $(14.2 \%)$ rated live pumpkins successful in raising profit margins.

The average rating of Easter gift plants (e. g. Easter lilies) was $2.50(S D=.97)$ (see Table 29). Easter gift plants were rated unsuccessful by three respondents (18.8\%). Four respondents (25.0\%) rated Easter gift plants slightly successful while seven respondents (43.8\%) rated Easter gift plants moderately successful. Two respondents (12.4\%) rated Easter gift plants successful in raising profit margins.

The average rating of Christmas decorations was $2.45(S D=1.14)$ (see Table 29). Christmas decorations were rated unsuccessful by six respondents (27.3\%). Five respondents (22.7\%) rated Christmas decorations slightly successful in raising profit margins while six respondents (27.3\%) rated Christmas decorations moderately successful. Five respondents (22.7\%) rated Christmas decorations successful.

The average rating of fresh-cut Christmas trees was $2.39(S D=1.09)$ (see Table 29). Fresh-cut Christmas trees were rated unsuccessful by five respondents (27.8\%). Four respondents (22.2\%) rated fresh cut Christmas trees slightly successful while six 
respondents $(33.3 \%)$ rated the trees moderately successful. Three respondents $(16.7 \%)$ rated fresh cut Christmas trees successful in raising profit margins.

The average rating of Halloween decorations was $2.16(S D=.77)($ see Table 29). Halloween decorations were rated unsuccessful in raising profit margins by three respondents (15.8\%). Eleven respondents (57.9\%) rated Halloween decorations slightly successful while four respondents (21.1\%) rated them moderately successful. One respondent (5.2\%) rated Halloween decorations successful.

The average rating of fresh-cut flowers for Valentine's Day was $2.14(S D=1.22)$ (see Table 29). Fresh-cut flowers for Valentine's Day were rated unsuccessful in raising profit margins by five respondents (15.6\%). Twenty seven respondents $(84.4 \%)$ rated fresh cut flowers for Valentine's Day slightly successful in raising profit margins

The average rating of artificial Christmas trees was $2.08(S D=1.12)$ (see Table 29). Five respondents (38.4\%) rated artificial Christmas trees unsuccessful in raising profit margins. Four respondents (30.8\%) rated artificial Christmas trees slightly successful while two respondents (15.4\%) rated them moderately successful. Two respondents (15.4\%) rated artificial Christmas trees successful.

The average rating of Easter decorations was $2.00(S D=.71)($ see Table 29). Easter decorations were rated unsuccessful at raising profit margins by four respondents (23.5\%). Nine respondents (53.0\%) rated Easter decorations slightly successful while four respondents (23.5\%) rated Easter decorations moderately successful.

The average rating of Thanksgiving decorations was $2.00(S D=.76)$ (see Table 29). Thanksgiving decorations were rated unsuccessful by four respondents $(26.7 \%)$ in raising profit margins. Seven respondents $(46.7 \%)$ rated Thanksgiving decorations 
slightly successful while four respondents (26.7\%) rated Thanksgiving decorations moderately successful.

The average rating of interior Christmas decorating services was $2.00(S D=1.32)$ (see Table 29). Interior Christmas decorating services were rated unsuccessful by five respondents (55.6\%). One respondent (11.1\%) rated interior Christmas decorating services slightly successful while one respondent (11.1\%) rated the service moderately successful. Two respondents (22.2\%) rated interior Christmas decorating services successful in raising profit margins.

The average rating of exterior Christmas decorating services was $1.75(S D=1.04)$ (see Table 29). Four respondents (50.0\%) rated Exterior Christmas decorating services unsuccessful. Three respondents (37.5\%) rated exterior Christmas decorating services slightly successful while one respondent $(12.5 \%)$ rated the service successful in raising profit margins. 
Table 29

Potential for Success in Raising Profit Margins-Holiday Merchandise or Services

\begin{tabular}{|c|c|c|c|c|c|c|c|c|c|c|}
\hline & \multicolumn{4}{|c|}{ Unsuccessful } & \multicolumn{6}{|c|}{$\begin{array}{l}\text { Slightly Moderately Successful } \\
\text { Successful Successful }\end{array}$} \\
\hline & $M$ & $S D$ & $N$ & $\%$ & $N$ & $\%$ & $N$ & $\%$ & $N$ & $\%$ \\
\hline $\begin{array}{l}\text { Custom made decorations } \\
\text { (e. g. wreaths, swags) }\end{array}$ & 2.90 & 1.04 & 2 & 9.5 & 6 & 28.6 & 5 & 23.8 & 8 & 38.1 \\
\hline $\begin{array}{l}\text { Christmas gift plants (e. g. } \\
\text { poinsettias) }\end{array}$ & 2.85 & 1.23 & 4 & 20.0 & 4 & 20.0 & 3 & 15.0 & 9 & 45.0 \\
\hline Painted poinsettias & 2.85 & 1.28 & 3 & 23.1 & 2 & 15.4 & 2 & 15.4 & 6 & 46.2 \\
\hline Container trees & 2.85 & 1.14 & 3 & 15.0 & 5 & 25.0 & 4 & 20.0 & 8 & 40.0 \\
\hline Balled and burlap trees & 2.65 & 1.06 & 2 & 11.8 & 7 & 41.2 & 3 & 17.6 & 5 & 29.4 \\
\hline Live pumpkins & 2.62 & .81 & 1 & 4.8 & 9 & 42.9 & 8 & 38.1 & 3 & 14.2 \\
\hline $\begin{array}{l}\text { Easter gift plants (e. g. Easter } \\
\text { lilies) }\end{array}$ & 2.50 & .97 & 3 & 18.8 & 4 & 25.0 & 7 & 43.8 & 2 & 12.4 \\
\hline Christmas decorations & 2.45 & 1.14 & 6 & 27.3 & 5 & 22.7 & 6 & 27.3 & 5 & 22.7 \\
\hline Fresh-cut Christmas trees & 2.39 & 1.09 & 5 & 27.8 & 4 & 22.2 & 6 & 33.3 & 3 & 16.7 \\
\hline Halloween decorations & 2.16 & .77 & 3 & 15.8 & 11 & 57.9 & 4 & 21.1 & 1 & 5.2 \\
\hline $\begin{array}{l}\text { Fresh cut flowers for } \\
\text { Valentine's Day }\end{array}$ & 2.14 & 1.22 & 5 & 15.6 & 27 & 84.4 & 0 & 0.0 & 0 & 0.0 \\
\hline Artificial Christmas trees & 2.08 & 1.12 & 5 & 38.4 & 4 & 30.8 & 2 & 15.4 & 2 & 15.4 \\
\hline Easter decorations & 2.00 & .71 & 4 & 23.5 & 9 & 53.0 & 4 & 23.5 & 0 & 0.0 \\
\hline Thanksgiving decorations & 2.00 & .76 & 4 & 26.7 & 7 & 46.7 & 4 & 26.7 & 0 & 0.0 \\
\hline $\begin{array}{l}\text { Interior Christmas decorating } \\
\text { services }\end{array}$ & 2.00 & 1.32 & 5 & 55.6 & 1 & 11.1 & 1 & 11.1 & 2 & 22.2 \\
\hline $\begin{array}{l}\text { Exterior Christmas decorating } \\
\text { services }\end{array}$ & 1.75 & 1.04 & 4 & 50.0 & 3 & 37.5 & 0 & 0.0 & 1 & 12.5 \\
\hline
\end{tabular}


Children's Activities. Using a scale of 1-4 with 1 being "unsuccessful" and 4 "successful" the average rating of plant their own plants to take home was $2.50(S D=$ 1.43) (see Table 30). Letting children plant their own plants to take home was rated unsuccessful in raising profit margins by four respondents (40.0\%). One respondent (10.0\%) rated letting children plant their own plants to take home slightly successful while one respondent $(10.0 \%)$ rated the activity moderately successful. Four respondents $(40.0 \%)$ rated the activity successful in raising profit margins.

The average rating of corn maze was $2.00(S D=.82)($ see Table 30$)$. Hosting a corn maze was rated unsuccessful by one respondent $(25.0 \%)$. Two respondents $(50.0 \%)$ rated a corn maze slightly successful. One respondent (25.0\%) rated a corn maze moderately successful.

The average rating of pony rides was $1.60(S D=.55)$ (see Table 30$)$. Giving pony rides was rated unsuccessful by four respondents (12.5\%) while 28 respondents (87.5\%) rated pony rides slightly successful in raising profit margins. The average rating of petting zoo was $1.50(S D=.58)$ (see Table 30$)$. Hosting a petting zoo was rated unsuccessful by two respondents $(50.0 \%)$ in raising profit margins. Two respondents $(50.0 \%)$ rated a petting zoo slightly successful.

The average rating of build and decorate a straw house was $1.50(S D=.71)$ (see Table 30). Building and decorating a straw house was rated unsuccessful by one respondent $(50.0 \%)$ and rated slightly successful by one respondent $(50.0 \%)$. The average rating of photos with Santa was $1.40(S D=.55)$ (see Table 30). Photos with Santa were rated unsuccessful by three respondents $(60.0 \%)$. Two respondents $(40.0 \%)$ rated photos with Santa slightly successful in raising profit margins. 
The average rating of haunted greenhouse was $1.33(S D=.58)$ (see Table 30$)$. Two respondents $(66.7 \%)$ rated a haunted greenhouse unsuccessful in raising profit margins while one respondent (33.3\%) rated a haunted greenhouse slightly successful. The average rating of photos with Easter Bunny was $1.25(S D=.50)$ (see Table 30$)$. Photos with the Easter Bunny were rated unsuccessful by three respondents $(75.0 \%)$. One respondent $(25.0 \%)$ rated Photos with the Easter Bunny slightly successful in raising profit margins. The average rating of sell Easter pets was $1.00(S D=.00)$ (see Table 30$)$. Selling Easter pets (e. g. chicks, ducks, bunnies) was rated unsuccessful by two respondents $(100.0 \%)$. 
Table 30

Potential for Success in Raising Profit Margins-Children's Activities

Unsuccessful Slightly Moderately Successful Successful Successful

$\begin{array}{llllllllll}M & S D & N & \% & N & \% & N & \% & N & \%\end{array}$

\begin{tabular}{|c|c|c|c|c|c|c|c|c|c|c|}
\hline $\begin{array}{l}\text { Plant their own plants to } \\
\text { take home }\end{array}$ & 2.50 & 1.43 & 4 & 40.0 & 1 & 10.0 & 1 & 10.0 & 4 & 40.0 \\
\hline Corn maze & 2.00 & .82 & 1 & 25.0 & 2 & 50.0 & 1 & 25.0 & 0 & 0.0 \\
\hline Pony rides & 1.60 & .55 & 4 & 12.5 & 28 & 87.5 & 0 & 0.0 & 0 & 0.0 \\
\hline Petting zoo & 1.50 & .58 & 2 & 50.0 & 2 & 50.0 & 0 & 0.0 & 0 & 0.0 \\
\hline $\begin{array}{l}\text { Build and decorate a straw } \\
\text { house }\end{array}$ & 1.50 & .71 & 1 & 50.0 & 1 & 50.0 & 0 & 0.0 & 0 & 0.0 \\
\hline Photos with Santa & 1.40 & .55 & 3 & 60.0 & 2 & 40.0 & 0 & 0.0 & 0 & 0.0 \\
\hline Haunted greenhouse & 1.33 & .58 & 2 & 66.7 & 1 & 33.3 & 0 & 0.0 & 0 & 0.0 \\
\hline Photos with Easter Bunny & 1.25 & .50 & 3 & 75.0 & 1 & 25.0 & 0 & 0.0 & 0 & 0.0 \\
\hline $\begin{array}{l}\text { Sell Easter pets (e. g. } \\
\text { chicks, ducks, bunnies) }\end{array}$ & 1.00 & .00 & 2 & 100.0 & 0 & 0.0 & 0 & 0.0 & 0 & 0.0 \\
\hline
\end{tabular}

Advertisement. Using a scale of 1-4 with 1 being "unsuccessful" and 4 "successful" the average rating of television was $2.94(S D=1.06)$ (see Table 31$)$. Fourteen respondents (45.2\%) rated television unsuccessful in raising profit margins. Seventeen respondents $(54.8 \%)$ rated television slightly successful in raising profit margins.

The average rating of using a year round marketing program instead of seasonal was $2.91(S D=.94)$ (see Table 31$)$. Using a year round marketing program instead of a 
seasonal program was rated unsuccessful by one respondent $(9.1 \%)$. Two respondents $(18.2 \%)$ rated a year round marketing program slightly successful while five respondents (45.4\%) rated it moderately successful. Three respondents $(27.3 \%)$ rated a year round marketing program successful in raising profit margins.

The average rating of newspaper was $2.87(S D=.92)($ see Table 31). Three respondents $(13.1 \%)$ rated the newspaper unsuccessful in raising profit margins. Two respondents $(8.7 \%)$ rated the newspaper slightly successful while 13 respondents $(56.5 \%)$ rated the newspaper moderately successful. Five respondents $(21.7 \%)$ rated the newspaper successful in raising profit margins.

The average rating of radio was $2.84(S D=.77)$ (see Table 31$)$. The radio was rated unsuccessful by one respondent $(5.3 \%)$. Four respondents $(21.1 \%)$ rated the radio slightly successful while 11 respondents $(57.8 \%)$ rated the radio moderately successful. Three respondents $(15.8 \%)$ rated the radio successful in raising profit margins.

The average rating of sending periodic newsletters to customers was $2.71(S D=$ .91) (see Table 31). Two respondents (14.3\%) rated sending periodic newsletters to customers unsuccessful in raising profit margins. Two respondents $(14.3 \%)$ rated sending newsletters slightly successful while eight respondents (57.1\%) rated sending newsletters moderately successful. Two respondents $(14.3 \%)$ rated sending newsletters successful in raising profit margins.

The average rating of emailing customers new product updates was $2.63(S D=$ .92). Emailing customers new product updates was rated unsuccessful by one respondent (12.5\%). Two respondents $(25.0 \%)$ rated email slightly successful while four 
respondents $(50.0 \%)$ rated email moderately successful. One respondent $(12.5 \%)$ rated emailing customers new product updates successful in raising profit margins.

The average rating of utilizing a company website was $2.55(S D=.61)$ (see Table 31). Ten respondents $(50.0 \%)$ rated utilizing a company website slightly successful in raising profit margins. Nine respondents (45.0\%) rated using a website moderately successful while one respondent (5.0\%) rated using a website successful in raising profit margins.

Table 31

Potential for Success in Raising Profit Margins-Advertisement

\begin{tabular}{|c|c|c|c|c|c|c|c|c|c|c|}
\hline & \multicolumn{4}{|c|}{ Unsuccessful } & \multicolumn{2}{|c|}{$\begin{array}{l}\text { Slightly } \\
\text { Successful }\end{array}$} & \multicolumn{4}{|c|}{$\begin{array}{l}\text { Moderately Successful } \\
\text { Successful }\end{array}$} \\
\hline & $M$ & $S D$ & $N$ & $\%$ & $N$ & $\%$ & $N$ & $\%$ & $N$ & $\%$ \\
\hline Television & 2.94 & 1.06 & 14 & 45.2 & 17 & 54.8 & 0 & 0.0 & 0 & 0.0 \\
\hline $\begin{array}{l}\text { Using a year round marketing } \\
\text { program instead of seasonal }\end{array}$ & 2.91 & .94 & 1 & 9.1 & 2 & 18.2 & 5 & 45.4 & 3 & 27.3 \\
\hline Newspaper & 2.87 & .92 & 3 & 13.1 & 2 & 8.7 & 13 & 56.5 & 5 & 21.7 \\
\hline Radio & 2.84 & .77 & 1 & 5.3 & 4 & 21.1 & 11 & 57.8 & 3 & 15.8 \\
\hline $\begin{array}{l}\text { Sending periodic newsletters } \\
\text { to customers }\end{array}$ & 2.71 & .91 & 2 & 14.3 & 2 & 14.3 & 8 & 57.1 & 2 & 14.3 \\
\hline $\begin{array}{l}\text { Emailing customers new } \\
\text { product updates }\end{array}$ & 2.63 & .92 & 1 & 12.5 & 2 & 25.0 & 4 & 50.0 & 1 & 12.5 \\
\hline Utilizing a company website & 2.55 & .61 & 0 & 0.0 & 10 & 50.0 & 9 & 45.0 & 1 & 5.0 \\
\hline
\end{tabular}


Educational Seminars. Using a scale of 1-4 with 1 being "unsuccessful" and 4 "successful" the average rating of flower arranging was $2.71(S D=.76)$ (see Table 32). Flower arranging was rated slightly successful by three respondents (42.9\%). Three respondents (42.9\%) rated flower arranging moderately successful while one respondent $(14.2 \%)$ rated the seminar successful.

The average rating of planting was $2.46(S D=.78)$ (see Table 32$)$. Twelve respondents $(40.0 \%)$ rated planting unsuccessful while 18 respondents $(60.0 \%)$ rated the seminar slightly successful. The average rating of pruning was $2.45(S D=.82)$ (see Table 32). Pruning was rated unsuccessful by one respondent (9.1\%). Five respondents (45.4\%) rated pruning slightly successful while four respondents $(36.4 \%)$ rated the seminar moderately successful. One respondent $(9.1 \%)$ rated pruning successful.

The average rating of cooking with herbs was $2.30(S D=.84)($ see Table 32$)$. Cooking with herbs was rated unsuccessful in raising profit margins by one respondent (10.0\%). Five respondents $(50.0 \%)$ rated cooking with herbs slightly successful while three respondents (30.0\%) rated cooking with herbs moderately successful. One respondent $(10.0 \%)$ rated cooking with herbs successful.

The average rating of herbal health remedies was $2.40(S D=1.14)$ (see Table 32). Herbal health remedies was rated unsuccessful by one respondent (20.0\%). Two respondents $(40.0 \%)$ rated the seminar slightly successful while one respondent $(20.0 \%)$ rated it moderately successful. One respondent $(20.0 \%)$ rated herbal health remedies successful in raising profit margins.

The average rating of plants that attract birds was $2.36(S D=1.03)$ (see Table 32). Plants that attract birds was rated unsuccessful by three respondents (27.3\%). Two 
respondents $(18.2 \%)$ rated the seminar slightly successful while five respondents $(45.4 \%)$ rated the seminar moderately successful. One respondent $(9.1 \%)$ rated plants that attract birds successful in raising profit margins.

The average rating of plants that attract butterflies was $2.36(S D=1.03)$ (see Table 32). Plants that attract butterflies was rated unsuccessful by three respondents $(27.3 \%)$. Two respondents $(18.2 \%)$ rated the seminar slightly successful while five respondents (45.4\%) rated the seminar moderately successful. One respondent $(9.1 \%)$ rated the seminar on plants that attract butterflies successful in raising profit margins.

The average rating of drying and storing herbs was $2.33(S D=1.03)$ (see Table 32). Drying and storing herbs was rated unsuccessful by one respondent (16.7\%). Three respondents (49.9\%) rated drying and storing herbs slightly successful while one respondent (16.7\%) rated drying and storing herbs moderately successful. One respondent $(16.7 \%)$ rated the seminar successful.

The average rating of dividing perennials was $2.33(S D=1.00)$ (see Table 32). Dividing perennials was rated unsuccessful by two respondents $(22.2 \%)$. Three respondents $(33.3 \%)$ rated the seminar slightly successful while three respondents $(33.3 \%)$ rated the seminar moderately successful. One respondent $(11.1 \%)$ rated the seminar on dividing perennials successful in raising profit margins.

The average rating of gardening basics was $2.33(S D=.99)$ (see Table 32). Gardening basics was rated unsuccessful by three respondents $(25.0 \%)$. Three respondents $(25.0 \%)$ rated the seminar slightly successful while five respondents $(41.7 \%)$ rated it moderately successful. One respondent $(8.3 \%)$ rated the seminar successful in raising profit margins. 
The average rating of plants that repel pests (e. g. deer, rabbits, etc) was 2.29 (SD $=1.25)$ (see Table 32). Plants that repel pests were rated unsuccessful by two respondents $(28.6 \%)$. Three respondents $(42.8 \%)$ rated the seminar slightly successful while two respondents (28.6\%) rated the seminar successful in raising profit margins.

The average rating of preparing gardens for winter was $2.25(S D=1.28)$ (see Table 32). Preparing gardens for winter was rated unsuccessful by three respondents (37.5\%). Two respondents (25.0\%) rated the seminar slightly successful while one respondent $(12.5 \%)$ rated it moderately successful. Two respondents $(25.0 \%)$ rated the seminar successful in raising profit margins.

The average rating of dos and don'ts of mulch was $2.14(S D=1.07)$ (see Table 32). Dos and don'ts of mulch was rated unsuccessful by two respondents (28.6\%). Three respondents (42.8\%) rated the seminar slightly successful while one respondent (14.3\%) rated the seminar moderately successful. One respondent (14.3\%) rated the dos and don'ts of mulch successful in raising profit margins.

The average rating of identifying diseases was $1.83(S D=.75)$ (see Table 32). Two respondents (33.3\%) rated identifying diseases unsuccessful while three respondents $(50.0 \%)$ rated the seminar slightly successful. One respondent (16.7\%) rated identifying diseases successful in raising profit margins.

The average rating of gardening without pain was $1.75(S D=.50)$ (see Table 32$)$. Gardening without pain was rated unsuccessful by one respondent (25.0\%) while three respondents $(75.0 \%)$ rated the seminar slightly successful. The average rating of identifying insect pests was $1.60(S D=.55)$ (see Table 32$)$. Identifying insect pests was 
rated unsuccessful by two respondents $(40.0 \%)$ while three respondents $(60.0 \%)$ rated the seminar slightly successful.

The average rating of drying flowers was $1.50(S D=.55)$ (see Table 32). Drying flowers was rated unsuccessful by three respondents $(50.0 \%)$ while three respondents $(50.0 \%)$ rated the seminar slightly successful. The average rating of preserving birdhouses and feeders, using landscape lighting to accent gardens at night, maintaining deck wood and maintaining outdoor furniture was $1.33(S D=.58)$ (see Table 32).

Preserving birdhouses and feeders was rated unsuccessful by two respondents $(66.7 \%)$ while one respondent (33.3\%) rated the seminar slightly successful. Two respondents (66.7\%) rated using landscape lighting to accent gardens at night unsuccessful while one respondent (33.3\%) rated the seminar slightly successful. Maintaining deck wood was rated unsuccessful by two respondents $(66.7 \%)$ while one respondent $(33.3 \%)$ rated the seminar slightly successful. Two respondents $(66.7 \%)$ rated maintaining outdoor furniture unsuccessful while one respondent $(33.3 \%)$ rated the seminar slightly successful. 
Table 32

Potential for Success in Raising Profit Margins_Educational Seminars

\begin{tabular}{|c|c|c|c|c|c|c|c|c|c|c|}
\hline & \multicolumn{4}{|c|}{ Unsuccessful } & \multicolumn{6}{|c|}{$\begin{array}{l}\text { Slightly Moderately Successful } \\
\text { Successful Successful }\end{array}$} \\
\hline & $M$ & $S D$ & $N$ & $\%$ & $N$ & $\%$ & $N$ & $\%$ & $N$ & $\%$ \\
\hline Flower arranging & 2.71 & .76 & 0 & 0.0 & 3 & 42.9 & 3 & 42.9 & 1 & 14.2 \\
\hline Planting & 2.46 & .78 & 12 & 40.0 & 18 & 60.0 & 0 & 0.0 & 0 & 0.0 \\
\hline Pruning & 2.45 & .82 & 1 & 9.1 & 5 & 45.4 & 4 & 36.4 & 1 & 9.1 \\
\hline Cooking with herbs & 2.40 & .84 & 1 & 10.0 & 5 & 50.0 & 3 & 30.0 & 1 & 10.0 \\
\hline Herbal health remedies & 2.40 & 1.14 & 1 & 20.0 & 2 & 40.0 & 1 & 20.0 & 1 & 20.0 \\
\hline Plants that attract birds & 2.36 & 1.03 & 3 & 27.3 & 2 & 18.2 & 5 & 45.4 & 1 & 9.1 \\
\hline Plants that attract butterflies & 2.36 & 1.03 & 3 & 27.3 & 2 & 18.2 & 5 & 45.4 & 1 & 9.1 \\
\hline Drying and storing herbs & 2.33 & 1.03 & 1 & 16.7 & 3 & 49.9 & 1 & 16.7 & 1 & 16.7 \\
\hline Dividing perennials & 2.33 & 1.00 & 2 & 22.2 & 3 & 33.3 & 3 & 33.3 & 1 & 11.1 \\
\hline Gardening basics & 2.33 & .99 & 3 & 25.0 & 3 & 25.0 & 5 & 41.7 & 1 & 8.3 \\
\hline $\begin{array}{l}\text { Plants that repel pests (e. g. } \\
\text { deer, rabbits, etc) }\end{array}$ & 2.29 & 1.25 & 2 & 28.6 & 3 & 42.8 & 0 & 0.0 & 2 & 28.6 \\
\hline Preparing gardens for winter & 2.25 & 1.28 & 3 & 37.5 & 2 & 25.0 & 1 & 12.5 & 2 & 25.0 \\
\hline Dos and don'ts of mulch & 2.14 & 1.07 & 2 & 28.6 & 3 & 42.8 & 1 & 14.3 & 1 & 14.3 \\
\hline Identifying diseases & 1.83 & .75 & 2 & 33.3 & 3 & 50.0 & 1 & 16.7 & 0 & 0.0 \\
\hline Gardening without pain & 1.75 & .50 & 1 & 25.0 & 3 & 75.0 & 0 & 0.0 & 0 & 0.0 \\
\hline Identifying insect pests & 1.60 & .55 & 2 & 40.0 & 3 & 60.0 & 0 & 0.0 & 0 & 0.0 \\
\hline Drying flowers & 1.50 & .55 & 3 & 50.0 & 3 & 50.0 & 0 & 0.0 & 0 & 0.0 \\
\hline $\begin{array}{l}\text { Preserving birdhouses and } \\
\text { feeders }\end{array}$ & 1.33 & .58 & 2 & 66.7 & 1 & 33.3 & 0 & 0.0 & 0 & 0.0 \\
\hline
\end{tabular}


Table 32 (Continued)

Potential for Success in Raising Profit Margins_Educational Seminars

Unsuccessful Slightly Moderately Successful Successful Successful

\begin{tabular}{llllllllll}
\hline$M$ & $S D$ & $N$ & $\%$ & $N$ & $\%$ & $N$ & $\%$ & $N$ & $\%$ \\
\hline
\end{tabular}

Using landscape lighting to accent gardens at night

$\begin{array}{llllllllll}1.33 & .58 & 2 & 66.7 & 1 & 33.3 & 0 & 0.0 & 0 & 0.0\end{array}$

Maintaining deck wood

$\begin{array}{llllllllll}1.33 & .58 & 2 & 66.7 & 1 & 33.3 & 0 & 0.0 & 0 & 0.0\end{array}$

Maintaining outdoor furniture

$1.33 \quad .58$

Services for the Community. Using a scale of 1-4 with 1 being "unsuccessful" and 4 "successful" the average rating of donating plants to public areas was $2.19(S D=$ .83) (see Table 33). Donating plants to public areas was rated unsuccessful by three respondents (18.8\%). Eight respondents (50.0\%) rated the service slightly successful while four respondents $(25.0 \%)$ rated the service moderately successful. One respondent $(6.2 \%)$ rated donating plants to public areas successful in raising profit margins.

The average rating of local craft show was $2.17(S D=1.47)$ (see Table 33). Hosting a local craft show was rated unsuccessful by three respondents $(50.0 \%)$. One respondent (16.7\%) rated the service slightly successful while two respondents $(33.3 \%)$ rated hosting a local craft show successful.

The average rating of donating supplies to public areas (e. g. mulch) was 2.00 (SD $=.89$ ) (see Table 33). Donating supplies to public areas was rated unsuccessful by 21 respondents $(65.6 \%)$. Eleven respondents $(34.4 \%)$ rated the service slightly successful in raising profit margins. 
The average rating of farmers market with free space for vendors was $1.60(S D=$ 1.34) (see Table 33). Four respondents $(80.0 \%)$ rated hosting a farmers market with free space for vendors unsuccessful while one respondent $(20.0 \%)$ rated the service successful. The average rating of farmers market with paid space for vendors was 1.00 $(S D=.00)($ see Table 33$)$. Three respondents $(100.0 \%)$ rated hosting a farmers market with paid space for vendors unsuccessful in raising profit margins.

Table 33

Potential for Success in Raising Profit Margins_-Services for the Community

\begin{tabular}{|c|c|c|c|c|c|c|c|c|c|c|}
\hline & & & nsuc & essful & $\begin{array}{r}\text { Sli } \\
\text { Suc }\end{array}$ & $\begin{array}{l}\text { htly } \\
\text { essful }\end{array}$ & $\begin{array}{l}\text { Moc } \\
\text { Suc }\end{array}$ & $\begin{array}{l}\text { rately } \\
\text { essful }\end{array}$ & Suc & essful \\
\hline & $M$ & $S D$ & $N$ & $\%$ & $N$ & $\%$ & $N$ & $\%$ & $N$ & $\%$ \\
\hline $\begin{array}{l}\text { Donation of plants to } \\
\text { public areas }\end{array}$ & 2.19 & .83 & 3 & 18.8 & 8 & 50.0 & 4 & 25.0 & 1 & 6.2 \\
\hline Local craft show & 2.17 & 1.47 & 3 & 50.0 & 1 & 16.7 & 0 & 0.0 & 2 & 33.3 \\
\hline $\begin{array}{l}\text { Donation of supplies to } \\
\text { public areas (e. g. mulch) }\end{array}$ & 2.00 & .89 & 21 & 65.6 & 11 & 34.4 & 0 & 0.0 & 0 & 0.0 \\
\hline $\begin{array}{l}\text { Farmers market with free } \\
\text { space for vendors }\end{array}$ & 1.60 & 1.34 & 4 & 80.0 & 0 & 0.0 & 0 & 0.0 & 1 & 20.0 \\
\hline $\begin{array}{l}\text { Farmers market with paid } \\
\text { space for vendors }\end{array}$ & 1.00 & .00 & 3 & 100.0 & 0 & 0.0 & 0 & 0.0 & 0 & 0.0 \\
\hline
\end{tabular}

Services for Customers. Using a scale of 1-4 with 1 being "unsuccessful" and 4 "successful" the average rating of custom container design (e. g. hanging baskets, patio planters) was $3.52(S D=3.52)$ (see Table 34$)$. Custom container design was rated slightly successful by two respondents $(9.5 \%)$. Six respondents $(28.6 \%)$ rated the service 
moderately successful while 13 respondents $(61.9 \%)$ rated the service successful in raising profit margins.

The average rating of customer designed containers grown by the greenhouse until pick up date was $3.44(S D=.73)$ (see Table 34$)$. Customer designed containers grown by the greenhouse until pickup date was rated slightly successful by two respondents $(12.5 \%)$. Five respondents $(31.3 \%)$ rated the service moderately successful while nine respondents $(56.2 \%)$ rated the service successful in returning customers to the store.

The average rating of landscape installation was $3.39(\mathrm{SD}=.98)$ (see Table 34). Landscape installation was rated unsuccessful by two respondents $(11.1 \%)$. Five respondents $(27.8 \%)$ rated the service moderately successful while 11 respondents (61.1\%) rated landscape installation successful in raising profit margins.

The average rating of landscape design was $3.26(S D=.87)$ (see Table 34$)$. Landscape design was rated unsuccessful by one respondent (5.3\%). Two respondents $(10.5 \%)$ rated the service slightly successful while seven respondents $(36.8 \%)$ rated the service moderately successful. Nine respondents (47.4\%) rated landscape design successful in raising profit margins.

The average rating of pruning shrubs was $3.15(S D=.99)$ (see Table 34). Pruning shrubs was rated unsuccessful by one respondent (7.6\%). Two respondents $(15.4 \%)$ rated the service slightly successful while four respondents $(30.8 \%)$ rated the service moderately successful. Six respondents $(46.2 \%)$ rated pruning shrubs successful in raising profit margins. 
The average rating of pruning trees was $2.89(S D=1.27)$ (see Table 34). Pruning trees was rated unsuccessful by two respondents $(22.2 \%)$. One respondent $(11.1 \%)$ rated the service slightly successful while two respondents $(22.2 \%)$ rated the service moderately successful. Four respondents (44.4\%) rated pruning trees successful in raising profit margins.

The average rating of providing rental plants for weddings was $2.36(S D=1.03)$ (see Table 34). Providing rental plants for weddings was rated unsuccessful by three respondents $(27.3 \%)$. Two respondents $(18.2 \%)$ rated the service slightly successful while five respondents $(45.4 \%)$ rated the service moderately successful. One respondent $(9.1 \%)$ rated rental plants for weddings successful in raising profit margins.

The average rating of water garden design was $2.33(\mathrm{SD}=1.23)$ (see Table 34$)$. Water garden design was rated unsuccessful by four respondents (33.3\%). Three respondents $(25.0 \%)$ rated the service slightly successful while two respondents $(16.7 \%)$ rated the service moderately successful. Three respondents $(25.0 \%)$ rated water garden design successful in raising profit margins.

The average rating of water garden installation was $2.13(S D=1.36)$ (see Table 34). Water garden installation was rated unsuccessful by four respondents $(50.0 \%)$. One respondent (12.5\%) rated the service slightly successful while one respondent $(12.5 \%)$ rated the service moderately successful. Two respondents $(25.0 \%)$ rated water garden installation successful in raising profit margins.

The average rating of floral design for weddings (e. g. corsages) was $2.13(S D=$ 1.25) (see Table 34). Floral design for weddings (e. g. corsages) was rated unsuccessful 
by four respondents $(50.0 \%)$. Three respondents $(37.5 \%)$ rated the service moderately successful while one respondent (12.5\%) rated the service successful.

Table 34

Potential for Success in Raising Profit Margins-Services for Customers

Unsuccessful Slightly Moderately Successful Successful Successful

$\begin{array}{llllllllll}M & S D & N & \% & N & \% & N & \% & N & \%\end{array}$

Custom container design (e.

g. hanging baskets, patio planters)

$\begin{array}{llllllllll}3.52 & .68 & 0 & 0.0 & 2 & 9.5 & 6 & 28.6 & 13 & 61.9\end{array}$

Customer designed containers - greenhouse grows until pickup date $\begin{array}{llllllllll}3.44 & .73 & 0 & 0.0 & 2 & 12.5 & 5 & 31.3 & 9 & 56.2\end{array}$

Landscape installation $\begin{array}{llllllllll}3.39 & .98 & 2 & 11.1 & 0 & 0.0 & 5 & 27.8 & 11 & 61.1\end{array}$

Landscape design

$\begin{array}{llllllllll}3.26 & .87 & 1 & 5.3 & 2 & 10.5 & 7 & 36.8 & 9 & 47.4\end{array}$

Pruning shrubs

$\begin{array}{llllllllll}3.15 & .99 & 1 & 7.6 & 2 & 15.4 & 4 & 30.8 & 6 & 46.2\end{array}$

Pruning trees

$\begin{array}{llllllllll}2.89 & 1.27 & 2 & 22.2 & 1 & 11.1 & 2 & 22.2 & 4 & 44.4\end{array}$

$\begin{array}{lllllllllll}\text { Rental plants for weddings } & 2.36 & 1.03 & 3 & 27.3 & 2 & 18.2 & 5 & 45.4 & 1 & 9.1\end{array}$

Water garden design

$\begin{array}{llllllllll}2.33 & 1.23 & 4 & 33.3 & 3 & 25.0 & 2 & 16.7 & 3 & 25.0\end{array}$

Water garden installation

$\begin{array}{llllllllll}2.13 & 1.36 & 4 & 50.0 & 1 & 12.5 & 1 & 12.5 & 2 & 25.0\end{array}$

Floral design for weddings

(e. g. corsages)

$\begin{array}{llllllllll}2.13 & 1.25 & 4 & 50.0 & 0 & 0.0 & 3 & 37.5 & 1 & 12.5\end{array}$

$\begin{array}{lllllllllll}\text { Lawn equipment repair } & 1.75 & 1.50 & 2 & 6.3 & 30 & 93.7 & 0 & 0.0 & 0 & 0.0\end{array}$

$\begin{array}{lllllllllll}\text { Rental plants for offices } & 1.50 & 1.00 & 3 & 75.0 & 0 & 0.0 & 1 & 25.0 & 0 & 0.0\end{array}$ 
The average rating of lawn equipment repair was $1.75(S D=1.50)$ (see Table 34). Lawn equipment repair was rated unsuccessful by two respondents (6.3\%). Thirty respondents $(93.7 \%)$ rated the service slightly successful in raising profit margins.

The average rating of rental plants for offices was $1.50(S D=1.00)$ (see Table 34). Rental plants for offices was rated unsuccessful by three respondents (75.0\%) while one respondent $(25.0 \%)$ rated the service moderately successful.

Lines of Plant Material. Using a scale of 1-4 with 1 being "unsuccessful" and 4 "successful" the average rating of larger sizes of annuals was $3.57(S D=.60)$ (see Table 35). Larger sizes of annuals were rated slightly successful by one respondent (4.8\%). Seven respondents (33.3\%) rated larger sizes of annuals moderately successful while 13 respondents $(61.9 \%)$ rated larger sizes of annuals successful.

The average rating of summer annuals (e. g. wave petunias) was $3.46(S D=.81)$ (see Table 35). Summer annuals were rated unsuccessful by one respondent (3.8\%). Two respondents (7.7\%) rated summer annuals slightly successful while seven respondents $(26.9 \%)$ rated summer annuals moderately successful. Sixteen respondents $(61.5 \%)$ rated summer annuals successful in raising profit margins.

The average rating of perennials was $3.42(S D=.76)$ (see Table 35). Perennials were rated unsuccessful by one respondent (3.8\%). One respondent (3.8\%) rated perennials slightly successful while 10 respondents (38.5\%) rated perennials moderately successful. Fourteen respondents (53.8\%) rated perennials successful in raising profit margins.

The average rating of summer blooming perennials was $3.42(S D=.70)$ (see Table 35). Summer blooming perennials were rated slightly successful by three 
respondents (11.5\%). Nine respondents (34.6\%) rated summer blooming perennials moderately successful while 14 respondents (53.8\%) rated summer blooming perennials successful.

The average rating of larger sizes of perennials was $3.32(S D=.84)$ (see Table 35). Larger sizes of perennials were rated unsuccessful by one respondent (4.5\%). Two respondents $(9.1 \%)$ rated larger sizes of perennials slightly successful while eight respondents (36.4\%) rated them moderately successful. Eleven respondents (50.0\%) rated larger sizes of perennials successful.

The average rating of outdoor container gardens was $3.30(\mathrm{SD}=.70)$ (see Table 35). Outdoor container gardens were rated slightly successful by three respondents $(13.0 \%)$. Ten respondents $(43.5 \%)$ rated outdoor container gardens moderately successful while 10 respondents $(43.5 \%)$ rated them successful.

The average rating of cool weather annuals (e. g. pansies) was $3.25(S D=.85)$ (see Table 35). Cool weather annuals were rated unsuccessful in raising profit margins by one respondent (4.2\%). Three respondents (12.5\%) rated cool weather annuals slightly successful while nine respondents (37.5\%) rated cool weather annuals moderately successful. Eleven respondents $(45.8 \%)$ rated cool weather annuals successful in raising profit margins.

The average rating of fall blooming ornamental grasses was $3.00(S D=.95)$ (see Table 35). Fall blooming ornamental grasses were rated unsuccessful by two respondents (8.7\%). Four respondents (17.4\%) rated fall blooming ornamental grasses slightly successful while nine respondents (39.1\%) rated them moderately successful. Eight 
respondents $(34.8 \%)$ rated fall blooming ornamental grasses successful in raising profit margins.

The average rating of windowsill herb pots was $2.94(S D=.80)($ see Table 35$)$. Windowsill herb pots were rated slightly successful by six respondents (33.3\%). Seven respondents (38.9\%) rated windowsill herb pots moderately successful. Five respondents $(27.8 \%)$ rated windowsill herb pots successful in raising profit margins.

The average rating of house plants (e. g. African violets, ivy, etc) was $2.84(S D=$ 1.17) (see Table 35). House plants were rated unsuccessful by four respondents $(21.1 \%)$. Two respondents $(10.5 \%)$ rated house plants slightly successful in raising profit margins while six respondents (31.6\%) rated house plants moderately successful. Seven respondents $(36.8 \%)$ rated house plants successful.

The average rating of herb topiaries was $2.82(S D=.75)$ (see Table 35). Herb topiaries were rated slightly successful by four respondents (36.4\%). Five respondents $(45.5 \%)$ rated herb topiaries moderately successful while two respondents $(18.0 \%)$ rated herb topiaries successful.

The average rating of plant propagation supplies (e. g. seed potatoes, onion sets and garden seeds) was $2.82(S D=.95)$ (see Table 35). Plant propagation supplies were rated unsuccessful in raising profit margins by 17 respondents $(53.1 \%)$. Fifteen respondents $(46.9 \%)$ rated plant propagation supplies slightly successful.

The average rating of orchids was $2.77(S D=1.17)($ see Table 35$)$. Orchids were rated unsuccessful in raising profit margins by three respondents $(23.1 \%)$. One respondent (7.7\%) rated orchids slightly successful. Five respondents (38.4\%) rated orchids moderately successful while four respondents $(30.8 \%)$ rated orchids successful. 
The average rating of container evergreens for indoors was $2.56(S D=1.33)$ (see Table 35). Container evergreens for indoors were rated unsuccessful by three respondents $(33.3 \%)$. One respondent $(11.1 \%)$ rated container evergreens for indoors slightly successful while two respondents $(22.2 \%)$ rated them moderately successful. Three respondents (33.3\%) rated container evergreens for indoors successful.

The average rating of fresh cut flowers year round was $2.43(S D=1.13)$ (see Table 35). Fresh cut flowers year round were rated unsuccessful in returning customers to the store by two respondents $(28.6 \%)$. One respondent $(14.3 \%)$ rated fresh cut flowers year round slightly successful while three respondents $(42.8 \%)$ rated them moderately successful. One respondent (14.3\%) rated fresh cut flowers year round successful in raising profit margins.

The average rating of fall bulbs (e. g. tulips) was $2.31(S D=1.25)$ (see Table 35$)$. Fall bulbs were rated unsuccessful by six respondents (37.4\%). Three respondents $(18.8 \%)$ rated fall bulbs slightly successful while three respondents $(18.8 \%)$ rated fall bulbs moderately successful. Four respondents $(25.0 \%)$ rated fall bulbs successful in raising profit margins.

The average rating of tropical bulbs (e. g. caladium) was $2.29(S D=1.07)$ (see Table 35). Tropical bulbs were rated unsuccessful by four respondents (28.6\%). Four respondents $(28.6 \%)$ rated tropical bulbs slightly successful and four respondents $(28.6 \%)$ rated tropical bulbs moderately successful. Two respondents $(14.2 \%)$ rated tropical bulbs successful.

The average rating of spring bulbs (e. g. dahlias) was $2.00(S D=1.11)$ (see Table 35). Spring bulbs were rated unsuccessful by six respondents (42.8\%). Four respondents 
(28.6\%) rated spring bulbs slightly successful while two respondents (14.3\%) rated spring bulbs moderately successful. Two respondents (14.3\%) rated spring bulbs successful in raising profit margins.

Table 35

Potential for Success in Raising Profit Margins_Lines of Plant Material

\begin{tabular}{|c|c|c|c|c|c|c|c|c|c|c|}
\hline & \multicolumn{4}{|c|}{ Unsuccessful } & \multicolumn{2}{|c|}{$\begin{array}{l}\text { Slightly } \\
\text { Successful }\end{array}$} & \multicolumn{2}{|c|}{$\begin{array}{l}\text { Moderately } \\
\text { Successful }\end{array}$} & \multicolumn{2}{|c|}{ Successful } \\
\hline & $M$ & $S D$ & $N$ & $\%$ & $N$ & $\%$ & $N$ & $\%$ & $N$ & $\%$ \\
\hline Larger sizes of annuals & 3.57 & .60 & 0 & 0.0 & 1 & 4.8 & 7 & 33.3 & 13 & 61.9 \\
\hline $\begin{array}{l}\text { Summer annuals (e. g. } \\
\text { wave petunias) }\end{array}$ & 3.46 & .81 & 1 & 3.8 & 2 & 7.7 & 7 & 26.9 & 16 & 61.5 \\
\hline Perennials & 3.42 & .76 & 1 & 3.8 & 1 & 3.8 & 10 & 38.5 & 14 & 53.8 \\
\hline $\begin{array}{l}\text { Summer blooming } \\
\text { perennials }\end{array}$ & 3.42 & .70 & 0 & 0.0 & 3 & 11.5 & 9 & 34.6 & 14 & 53.8 \\
\hline Larger sizes of perennials & 3.32 & .84 & 1 & 4.5 & 2 & 9.1 & 8 & 36.4 & 11 & 50.0 \\
\hline Outdoor container gardens & 3.30 & .70 & 0 & 0.0 & 3 & 13.0 & 10 & 43.5 & 10 & 43.5 \\
\hline $\begin{array}{l}\text { Cool weather annuals (e. g. } \\
\text { pansies) }\end{array}$ & 3.25 & .85 & 1 & 4.2 & 3 & 12.5 & 9 & 37.5 & 11 & 45.8 \\
\hline $\begin{array}{l}\text { Fall blooming ornamental } \\
\text { grasses }\end{array}$ & 3.00 & .95 & 2 & 8.7 & 4 & 17.4 & 9 & 39.1 & 8 & 34.8 \\
\hline Windowsill herb pots & 2.94 & .80 & 0 & 0.0 & 6 & 33.3 & 7 & 38.9 & 5 & 27.8 \\
\hline $\begin{array}{l}\text { House plants (e. g. African } \\
\text { violets, ivy, etc) }\end{array}$ & 2.84 & 1.17 & 4 & 21.1 & 2 & 10.5 & 6 & 31.6 & 7 & 36.8 \\
\hline Herb topiaries & 2.82 & .75 & 0 & 0.0 & 4 & 36.4 & 5 & 45.5 & 2 & 18.1 \\
\hline $\begin{array}{l}\text { Plant propagation supplies } \\
\text { (e. g. seed potatoes, onion } \\
\text { sets and garden seeds) }\end{array}$ & 2.82 & .95 & 17 & 53.1 & 15 & 46.9 & 0 & 0.0 & 0 & 0.0 \\
\hline
\end{tabular}


Table 35 (Continued)

Potential for Success in Raising Profit Margins_Lines of Plant Material

\begin{tabular}{|c|c|c|c|c|c|c|c|c|c|c|}
\hline & \multicolumn{4}{|c|}{ Unsuccessful } & \multicolumn{2}{|c|}{$\begin{array}{l}\text { Slightly } \\
\text { Successful }\end{array}$} & \multicolumn{2}{|c|}{$\begin{array}{l}\text { Moderately } \\
\text { Successful }\end{array}$} & \multicolumn{2}{|c|}{ Successful } \\
\hline & $M$ & $S D$ & $N$ & $\%$ & $N$ & $\%$ & $N$ & $\%$ & $N$ & $\%$ \\
\hline Orchids & 2.77 & 1.17 & 3 & 23.1 & 1 & 7.7 & 5 & 38.4 & 4 & 30.8 \\
\hline $\begin{array}{l}\text { Container evergreens for } \\
\text { indoors }\end{array}$ & 2.56 & 1.33 & 3 & 33.3 & 1 & 11.1 & 2 & 22.2 & 3 & 33.3 \\
\hline $\begin{array}{l}\text { Fresh cut flowers year } \\
\text { round }\end{array}$ & 2.43 & 1.13 & 2 & 28.6 & 1 & 14.3 & 3 & 42.8 & 1 & 14.3 \\
\hline Fall bulbs (e. g. tulips) & 2.31 & 1.25 & 6 & 37.4 & 3 & 18.8 & 3 & 18.8 & 4 & 25.0 \\
\hline $\begin{array}{l}\text { Tropical bulbs (e. g. } \\
\text { caladium) }\end{array}$ & 2.29 & 1.07 & 4 & 28.6 & 4 & 28.6 & 4 & 28.6 & 2 & 14.2 \\
\hline Spring bulbs (e. g. dahlias) & 2.00 & 1.11 & 6 & 42.8 & 4 & 28.6 & 2 & 14.3 & 2 & 14.3 \\
\hline
\end{tabular}

Lines of Merchandise. Using a scale of 1-4 with 1 being "unsuccessful” and 4 "successful" the average rating of hard goods (e. g. pottery, statues, etc) was 3.25 ( $S D=$ .79) (see Table 36). Hard goods were rated slightly successful by four respondents (20.0\%). Seven respondents (35.0\%) rated hard goods moderately successful while nine respondents (45.0\%) rated hard goods successful in raising profit margins.

The average rating of wind chimes was $2.94(S D=1.18)$ (see Table 36). Wind chimes were rated unsuccessful by three respondents (18.8\%). Two respondents $(12.4 \%)$ rated wind chimes slightly successful while four respondents (25.0\%) rated wind chimes moderately successful. Seven respondents (43.8\%) rated wind chimes successful.

The average rating of hummingbird supplies was $2.63(S D=1.09)$ (see Table 36). Hummingbird supplies were rated unsuccessful by three respondents (18.8\%). Four 
respondents (25.0\%) rated hummingbird supplies slightly successful while five respondents (31.2\%) rated the supplies moderately successful. Four respondents $(25.0 \%)$ rated hummingbird supplies successful.

The average rating of garden flags was $2.57(S D=1.28)$ (see table 36). Garden flags were rated unsuccessful by four respondents (28.6\%). Three respondents $(21.4 \%)$ rated garden flags slightly successful while two respondents (14.3\%) rated garden flags moderately successful. Five respondents (35.7\%) rated garden flags successful in raising profit margins.

The average rating of wildlife/bird feeding supplies was $2.33(S D=1.29)$ (see Table 36). Wildlife/bird feeding supplies were rated unsuccessful by six respondents (40.0\%). Two respondents (13.3\%) rated wildlife/bird feeding supplies slightly successful while three respondents (20.0\%) rated the supplies moderately successful. Four respondents (26.7\%) rated wildlife/bird feeding supplies successful in raising profit margins.

The average rating of outdoor furniture and accessories was $2.29(S D=1.14)$ (see Table 36). Outdoor furniture and accessories were rated unsuccessful by four respondents $(28.6 \%)$. Five respondents $(35.7 \%)$ rated outdoor furniture and accessories slightly successful while two respondents (14.3\%) rated outdoor furniture and accessories moderately successful. Three respondents (21.4\%) rated outdoor furniture and accessories successful.

The average rating of candles and accessories was $2.21(S D=1.05)$ (see Table 36). Candles and accessories were rated unsuccessful by four respondents (28.6\%). Five respondents (35.7\%) rated candles and accessories slightly successful while three 
respondents $(21.4 \%)$ rated them moderately successful. Two respondents $(14.3 \%)$ rated candles and accessories successful.

The average rating of pet food and supplies was $2.20(S D=1.64)$. Pet food and supplies were rated unsuccessful by three respondents $(60.0 \%)$ while two respondents $(40.0 \%)$ rated them successful.

The average rating of lawn equipment (e. g. mowers, weed trimmers) was 2.00 $(S D=1.41)($ see Table 36$)$. Lawn equipment was rated unsuccessful by three respondents $(60.0 \%)$. One respondent $(20.0 \%)$ rated lawn equipment moderately successful while one respondent (20.0\%) rated lawn equipment successful.

The average rating of garden themed clothing was $1.80(S D=1.30)$ (see Table 36). Garden themed clothing was rated unsuccessful by three respondents $(60.0 \%)$. One respondent (20.0\%) rated garden themed clothing slightly successful while one respondent $(20.0 \%)$ rated garden themed clothing successful.

The average rating of other jams and jellies was $1.50(S D=1.00)$ (see Table 36). One respondent (33.3\%) rated other jams and jellies unsuccessful while two respondents (66.7\%) rated other jams and jellies slightly successful (see Table 40).

The average rating of local jams and jellies was $1.33(S D=.58)$ (see Table 36). Local jams and jellies were rated unsuccessful by two respondents $(66.7 \%)$ while one respondent (33.3\%) rated local jams and jellies slightly successful.

The average rating of local wine was $1.33(S D=.58)$ (see Table 36). Local wine was rated unsuccessful by two respondents (66.7\%) while one respondent $(33.3 \%)$ rated local wine slightly successful. The average rating of other wine, local honey, other honey and canning supplies was $1.25(S D=.50)$ (see Table 36$)$. Other wine was rated 
unsuccessful by three respondents $(75.0 \%)$ while one respondent rated other wine (25.0\%). Local honey was rated unsuccessful by three respondents $(75.0 \%)$ while one respondent $(25.0 \%)$ rated local honey as slightly successful. Other honey was rated unsuccessful by three respondents $(75.0 \%)$ while one respondent $(25.0 \%)$ rated other honey slightly successful. Canning supplies were rated unsuccessful by three respondents (75.0\%) while one respondent $(25.0 \%)$ rated canning supplies slightly successful.

Table 36

Potential for Success in Raising Profit Margins_Lines of Merchandise

\begin{tabular}{|c|c|c|c|c|c|c|c|c|c|c|}
\hline & & & asuc & essful & $\begin{array}{r}\text { Sli } \\
\text { Succ }\end{array}$ & $\begin{array}{l}\text { htly } \\
\text { essful }\end{array}$ & $\begin{array}{l}\text { Moc } \\
\text { Suc }\end{array}$ & $\begin{array}{l}\text { rately } \\
\text { essful }\end{array}$ & Suc & essful \\
\hline & $M$ & $S D$ & $N$ & $\%$ & $N$ & $\%$ & $N$ & $\%$ & $N$ & $\%$ \\
\hline $\begin{array}{l}\text { Hard goods (e. g. pottery, } \\
\text { statues, etc) }\end{array}$ & 3.25 & .79 & 0 & 0.0 & 4 & 20.0 & 7 & 35.0 & 9 & 45.0 \\
\hline Wind chimes & 2.94 & 1.18 & 3 & 18.8 & 2 & 12.4 & 4 & 25.0 & 7 & 43.8 \\
\hline Hummingbird supplies & 2.63 & 1.09 & 3 & 18.8 & 4 & 25.0 & 5 & 31.2 & 4 & 25.0 \\
\hline Garden flags & 2.57 & 1.28 & 4 & 28.6 & 3 & 21.4 & 2 & 14.3 & 5 & 35.7 \\
\hline $\begin{array}{l}\text { Wildlife/bird feeding } \\
\text { supplies }\end{array}$ & 2.33 & 1.29 & 6 & 40.0 & 2 & 13.3 & 3 & 20.0 & 4 & 26.7 \\
\hline $\begin{array}{l}\text { Outdoor furniture and } \\
\text { accessories }\end{array}$ & 2.29 & 1.14 & 4 & 28.6 & 5 & 35.7 & 2 & 14.3 & 3 & 21.4 \\
\hline Candles and accessories & 2.21 & 1.05 & 4 & 28.6 & 5 & 35.7 & 3 & 21.4 & 2 & 14.3 \\
\hline Pet food and supplies & 2.20 & 1.64 & 3 & 60.0 & 0 & 0.0 & 0 & 0.0 & 2 & 40.0 \\
\hline $\begin{array}{l}\text { Lawn equipment (e. g. } \\
\text { mowers, weed trimmers) }\end{array}$ & 2.00 & 1.41 & 3 & 60.0 & 0 & 0.0 & 1 & 20.0 & 1 & 20.0 \\
\hline Garden themed clothing & 1.80 & 1.30 & 3 & 60.0 & 1 & 20.0 & 0 & 0.0 & 1 & 20.0 \\
\hline
\end{tabular}


Table 36 (Continued)

Potential for Success in Raising Profit Margins_Lines of Merchandise

\begin{tabular}{|c|c|c|c|c|c|c|c|c|c|c|}
\hline & \multicolumn{4}{|c|}{ Unsuccessful } & \multicolumn{2}{|c|}{$\begin{array}{c}\text { Slightly } \\
\text { Successful }\end{array}$} & \multicolumn{2}{|c|}{$\begin{array}{l}\text { Moderately } \\
\text { Successful }\end{array}$} & \multicolumn{2}{|c|}{ Successful } \\
\hline & $M$ & $S D$ & $N$ & $\%$ & $N$ & $\%$ & $N$ & $\%$ & $N$ & $\%$ \\
\hline Other jams and jellies & 1.50 & 1.00 & 1 & 33.3 & 2 & 66.7 & 0 & 0.0 & 0 & 0.0 \\
\hline Local jams and jellies & 1.33 & .58 & 2 & 66.7 & 1 & 33.3 & 0 & 0.0 & 0 & 0.0 \\
\hline Local wine & 1.33 & .58 & 2 & 66.7 & 1 & 33.3 & 0 & 0.0 & 0 & 0.0 \\
\hline Other wine & 1.25 & .50 & 3 & 75.0 & 1 & 25.0 & 0 & 0.0 & 0 & 0.0 \\
\hline Local honey & 1.25 & .50 & 3 & 75.0 & 1 & 25.0 & 0 & 0.0 & 0 & 0.0 \\
\hline Other honey & 1.25 & .50 & 3 & 75.0 & 1 & 25.0 & 0 & 0.0 & 0 & 0.0 \\
\hline Canning supplies & 1.25 & .50 & 3 & 75.0 & 1 & 25.0 & 0 & 0.0 & 0 & 0.0 \\
\hline
\end{tabular}

Adjustments to Business or Location. Using a scale of 1-4 with 1 being "unsuccessful" and 4 "successful" the average rating of customer friendly staff was 3.81 $(S D=.63)($ see Table 37$)$. Customer friendly staff was rated unsuccessful by one respondent (3.8\%). Two respondents $(7.7 \%)$ rated adjustment moderately successful. Twenty three respondents $(88.5 \%)$ rated customer friendly staff successful in raising profit margins.

The average rating of knowledgeable and experienced staff was $3.74(S D=.71)$ (see Table 37). Knowledgeable and experienced staff was rated unsuccessful by one respondent (3.7\%). One respondent (3.7\%) rated the adjustment slightly successful while two respondents $(7.4 \%)$ rated knowledgeable and experienced staff moderately 
successful. Twenty three respondents $(85.2 \%)$ rated the adjustment successful in raising profit margins.

The average rating of growing your own plants to allow for diverse product mix was $3.70(S D=.57)$ (see Table 37$)$. Growing your own plants to allow for diverse product mix was rated slightly successful by one respondent (5.0\%). Four respondents $(20.0 \%)$ rated the adjustment moderately successful while 15 respondents $(75.0 \%)$ rated it successful.

The average rating of raise prices on all items was $3.63(S D=.62)$ (see Table 37$)$. One respondent $(6.2 \%)$ rated raising prices on all items slightly successful while four respondents $(25.0 \%)$ rated the adjustment moderately successful. Eleven respondents $(68.8 \%)$ rated raising prices on all items successful in raising profit margins.

The average rating of display gardens was $3.47(S D=.62)$ (see Table 37). Display gardens were rated unsuccessful by one respondent $(5.9 \%)$. Seven respondents (41.1\%) rated display gardens moderately successful while nine respondents $(52.9 \%)$ rated display gardens successful in raising profit margins.

The average rating of establish a "greeter" at entrance was $3.46(S D=.66)$ (see Table 37). Establishing a "greeter" at the entrance was rated slightly successful by one respondent (7.7\%). Five respondents $(38.5 \%)$ rated the adjustment moderately successful while seven respondents (53.8\%) rated the adjustment successful.

The average rating of neat and clean aisles and displays was $3.40(S D=.87)($ see Table 37). Neat and clean aisles and displays were rated unsuccessful by one respondent (4.0\%). Three respondents $(12.0 \%)$ rated the adjustment slightly successful while six 
respondents $(24.0 \%)$ rated moderately successful. Fifteen respondents $(60.0 \%)$ rated neat and clean aisles and displays successful.

The average rating of add a covered shopping area for year round sales was 3.23 $(S D=1.09)$ (see Table 37$)$. Two respondents $(15.4 \%)$ rated adding a covered shopping area for year round sales unsuccessful. Four respondents $(30.8 \%)$ rated the adjustment moderately successful while seven respondents $(53.8 \%)$ rated it successful in raising profit margins.

The average rating of hire a certified horticulturist was $3.18(S D=1.17)$ (see Table 37). Hiring a certified horticulturist was rated unsuccessful by two respondents $(18.2 \%)$. Three respondents $(27.3 \%)$ rated the adjustment moderately successful while six respondents $(54.5 \%)$ rated hiring a certified horticulturist successful.

The average rating of establish warranty for plant material was $2.89(S D=.81)$ (see Table 37). One respondent (5.3\%) rated establishing a warranty for plant material unsuccessful. Four respondents $(21.1 \%)$ rated the adjustment slightly successful while 10 respondents (52.5\%) rated the adjustment moderately successful. Four respondents $(21.1 \%)$ rated establishing a warranty for plant material successful in raising profit margins.

The average rating of become bonded was $2.63(S D=1.41)$ (see Table 37$)$. Becoming bonded was rated unsuccessful by seven respondents $(24.1 \%)$ while 22 respondents $(75.9 \%)$ rated the adjustment slightly successful. The average rating of add a coffee house was $2.00(S D=1.00)$ (see Table 37). Adding a coffee house was rated unsuccessful by two respondents $(40.0 \%)$ while one respondent $(20.0 \%)$ rated the 
adjustment slightly successful. Two respondents $(40.0 \%)$ rated adding a coffee house moderately successful.

Table 37

Potential for Success in Raising Profit Margins-Adjustments to Business or Location

\begin{tabular}{lcccccccccccc}
\hline & \multicolumn{1}{c}{ Unsuccessful } & \multicolumn{1}{c}{ Slightly } & \multicolumn{1}{c}{ Moderately } \\
& \multicolumn{1}{c}{ Successful } & Successful & \\
& $M$ & $S D$ & $N$ & $\%$ & $N$ & $\%$ & $N$ & $\%$ & $N$ & $\%$ \\
& 3.81 & .63 & 1 & 3.8 & 0 & 0.0 & 2 & 7.7 & 23 & 88.5 \\
$\begin{array}{l}\text { Customer friendly staff } \\
\begin{array}{l}\text { Knowledgeable and } \\
\text { experienced staff }\end{array}\end{array}$ & 3.74 & .71 & 1 & 3.7 & 1 & 3.7 & 2 & 7.4 & 23 & 85.2
\end{tabular}

Growing your own plants to allow for diverse product mix

$\begin{array}{llllllllll}3.70 & .57 & 0 & 0.0 & 1 & 5.0 & 4 & 20.0 & 15 & 75.0 \\ 3.63 & .62 & 0 & 0.0 & 1 & 6.2 & 4 & 25.0 & 11 & 68.8 \\ 3.47 & .62 & 0 & 0.0 & 1 & 5.9 & 7 & 41.1 & 9 & 52.9 \\ 3.46 & .66 & 0 & 0.0 & 1 & 7.7 & 5 & 38.5 & 7 & 53.8\end{array}$

Neat and clean aisles and displays

$\begin{array}{llllllllll}3.40 & .87 & 1 & 4.0 & 3 & 12.0 & 6 & 24.0 & 15 & 60.0\end{array}$

Add a covered shopping area for year round sales

$\begin{array}{llllllllll}3.23 & 1.09 & 2 & 15.4 & 0 & 0.0 & 4 & 30.8 & 7 & 53.8\end{array}$

Hire a certified

horticulturist

$\begin{array}{llllllllll}3.18 & 1.17 & 2 & 18.2 & 0 & 0.0 & 3 & 27.3 & 6 & 54.5\end{array}$

Establish warranty for plant

material

Become bonded

Add a coffee house

Convert greenhouse to banquet hall during nonpeak months

Add a bakery

Add an ice cream shop $\begin{array}{llllllllll}2.89 & .81 & 1 & 5.3 & 4 & 21.1 & 10 & 52.5 & 4 & 21.1\end{array}$

$\begin{array}{llllllllll}2.63 & 1.41 & 7 & 24.1 & 22 & 75.9 & 0 & 0.0 & 0 & 0.0\end{array}$

$\begin{array}{llllllllll}2.00 & 1.00 & 2 & 40.0 & 1 & 20.0 & 2 & 40.0 & 0 & 0.0\end{array}$

$\begin{array}{llllllllll}1.75 & .96 & 2 & 50.0 & 1 & 25.0 & 1 & 25.0 & 0 & 0.0\end{array}$

$\begin{array}{llllllllll}1.67 & 1.16 & 2 & 66.7 & 0 & 0.0 & 1 & 33.3 & 0 & 0.0\end{array}$

$\begin{array}{llllllllll}1.67 & 1.16 & 2 & 66.7 & 0 & 0.0 & 1 & 33.3 & 0 & 0.0\end{array}$ 
The average rating of convert greenhouse to banquet hall during non-peak months was $1.75(S D=.96)$ (see Table 37). Converting the greenhouse to a banquet hall during non-peak months was rated unsuccessful by two respondents $(50.0 \%)$. One respondent $(25.0 \%)$ rated the adjustment slightly successful and one respondent $(25.0 \%)$ rated it moderately successful.

The average rating of add a bakery and add an ice cream shop was $1.67(S D=$ 1.16) (see Table 37). Two respondents $(66.7 \%)$ rated adding a bakery unsuccessful while one respondent (33.3\%) rated adding a bakery moderately successful. Adding an ice cream shop was rated unsuccessful by two respondents $(66.7 \%)$ while one respondent $(33.3 \%)$ rated it moderately successful.

Customer Appreciation. Using a scale of 1-4 with 1 being "unsuccessful" and 4 "successful" the average rating of seasonally dated coupons was $3.06(S D=.83)$ (see Table 38). Seasonally dated coupons were rated unsuccessful in raising profit margins by one respondent $(5.9 \%)$. Two respondents $(11.8 \%)$ rated seasonally dated coupons slightly successful while nine respondents $(52.9 \%)$ rated them moderately successful. Five respondents (29.4\%) rated seasonally dated coupons successful.

The average rating of percent-off sale events was $2.76(S D=.94)$ (see Table 38). Percent-off sale events were rated unsuccessful by two respondents (9.5\%). Six respondents $(28.6 \%)$ rated percent-off sale events slightly successful while eight respondents (38.1\%) rated the event moderately successful. Five respondents $(23.8 \%)$ rated percent-off sale events successful in raising profit margins.

Using a scale of 1-4 with 1 being "unsuccessful" and 4 "successful" the average rating of sell gift certificates at 20 percent off during non-peak months was $2.71(S D=$ 
.95) (see Table 38). Selling gift certificates at 20 percent off during non-peak months was rated unsuccessful by one respondent $(14.3 \%)$. One respondent $(14.3 \%)$ rated this method of customer appreciation slightly successful while four respondents (57.1\%) rated it moderately successful. One respondent (14.3\%) rated selling gift certificates at 20 percent off successful in raising profit margins.

The average rating of spend $\$ 50$ get free gift was $2.70(S D=.68)$ (see Table 38). Four respondents $(40.0 \%)$ rated a spend $\$ 50$ get free gift event slightly successful while five respondents (50.0\%) rated it moderately successful. One respondent (10.0\%) rated this method of customer appreciation successful.

The average rating of holiday specials on corresponding plants and accessories was $2.60(S D=1.17)($ see Table 38$)$. Two respondents $(20.0 \%)$ rated holiday specials on corresponding plants and accessories (i.e. Memorial Day: red, white and blue) unsuccessful. Three respondents (30.0\%) rated holiday specials on corresponding plants and accessories slightly successful while two respondents (20.0\%) rated it moderately successful. Three respondents (30.0\%) rated holiday specials on corresponding plants and accessories successful in raising profit margins.

The average rating of offer extra stamps on loyalty cards in non-peak months was $2.43(S D=.79)$ (see Table 38$)$. Offering extra stamps on loyalty cards in non-peak months was rated unsuccessful by one respondent (14.3\%). Two respondents (28.6\%) rated extra loyalty stamps slightly successful while four respondents (57.1\%) rated it moderately successful. The average rating of offering specials to customers in costume on Halloween was $1.67(S D=1.16)$ (see Table 38). Offering specials to customers in 
costume on Halloween was rated unsuccessful by two respondents $(66.7 \%)$ while one respondent (33.3\%) rated it moderately successful.

Table 38

Potential for Success in Raising Profit Margins_-Customer Appreciation

\begin{tabular}{|c|c|c|c|c|c|c|c|c|c|c|}
\hline & \multicolumn{4}{|c|}{ Unsuccessful } & \multicolumn{2}{|c|}{$\begin{array}{l}\text { Slightly } \\
\text { Successful }\end{array}$} & \multicolumn{4}{|c|}{$\begin{array}{l}\text { Moderately Successful } \\
\text { Successful }\end{array}$} \\
\hline & $M$ & $S D$ & $N$ & $\%$ & $N$ & $\%$ & $N$ & $\%$ & $N$ & $\%$ \\
\hline Seasonally dated coupons & 3.06 & .83 & 1 & 5.9 & 2 & 11.8 & 9 & 52.9 & 5 & 29.4 \\
\hline Percent-off sale events & 2.76 & .94 & 2 & 9.5 & 6 & 28.6 & 8 & 38.1 & 5 & 23.8 \\
\hline $\begin{array}{l}\text { Sell gift certificates at } 20 \\
\text { percent off during non-peak } \\
\text { months }\end{array}$ & 2.71 & .95 & 1 & 14.3 & 1 & 14.3 & 4 & 57.1 & 1 & 14.3 \\
\hline Spend $\$ 50$ get free gift & 2.70 & .68 & 0 & 0.0 & 4 & 40.0 & 5 & 50.0 & 1 & 10.0 \\
\hline $\begin{array}{l}\text { Holiday specials on } \\
\text { corresponding plants and } \\
\text { accessories i.e. Memorial } \\
\text { Day: red, white and blue }\end{array}$ & 2.60 & 1.17 & 2 & 20.0 & 3 & 30.0 & 2 & 20.0 & 3 & 30.0 \\
\hline $\begin{array}{l}\text { Offer extra stamps on loyalty } \\
\text { cards in non-peak months }\end{array}$ & 2.43 & .79 & 1 & 14.3 & 2 & 28.6 & 4 & 57.1 & 0 & 0.0 \\
\hline $\begin{array}{l}\text { Offer specials to customers in } \\
\text { costume on Halloween }\end{array}$ & 1.67 & 1.16 & 2 & 66.7 & 0 & 0.0 & 1 & 33.3 & 0 & 0.0 \\
\hline
\end{tabular}

Practices Garden Centers Will Use in the Future

Participants were asked to consider each practice in the questionnaire and decide whether or not they would use it in their garden center in the future. Respondents were asked to select yes or no to reflect their answer. 
Holiday Merchandise or Services. Twenty-one respondents (84.0\%) will use live pumpkins in the future. Custom made decorations (e. g. wreaths, swags) will be used by 18 respondents $(81.8 \%)$. Container trees will be used by 16 respondents $(80.0 \%)$.

Halloween decorations will be used by 19 respondents (76.0\%).

Christmas gift plants (e. g. poinsettias) will be used by 15 respondents (75.0\%). Balled and burlap trees will be used by 14 respondents (73.7\%). Easter gift plants (e. g. Easter lilies) will be used by 13 respondents (72.2\%). Christmas decorations will be used by 17 respondents (70.8\%). Thanksgiving decorations will be used by 14 respondents $(70.0 \%)$.

Fifteen respondents (68.2\%) will use Easter decorations in the future. Fourteen respondents (66.7\%) will use fresh-cut Christmas trees. Painted poinsettias will be used by eight respondents (53.3\%). Six respondents (37.5\%) will use artificial Christmas trees. Interior Christmas decorating services will be used by five respondents (35.7\%). Exterior Christmas decorating services will be used by three respondents (21.4\%). Five respondents (15.6\%) will use fresh cut flowers for Valentine's Day (see Table 43). 
Table 39

Practices Garden Centers Will Use in the Future-Holiday Merchandise or Services

\begin{tabular}{|c|c|c|c|c|}
\hline & \multicolumn{2}{|c|}{ Yes } & \multicolumn{2}{|c|}{ No } \\
\hline & $N$ & $\%$ & $N$ & $\%$ \\
\hline Live pumpkins & 21 & 84.0 & 4 & 16.0 \\
\hline Custom made decorations (e. g. wreaths, swags) & 18 & 81.8 & 4 & 18.2 \\
\hline Container trees & 16 & 80.0 & 4 & 20.0 \\
\hline Halloween decorations & 19 & 76.0 & 6 & 24.0 \\
\hline Christmas gift plants (e. g. poinsettias) & 15 & 75.0 & 5 & 25.0 \\
\hline Balled and burlap trees & 14 & 73.7 & 5 & 26.3 \\
\hline Easter gift plants (e. g. Easter lilies) & 13 & 72.2 & 5 & 27.8 \\
\hline Christmas decorations & 17 & 70.8 & 7 & 29.2 \\
\hline Thanksgiving decorations & 14 & 70.0 & 6 & 30.0 \\
\hline Easter decorations & 15 & 68.2 & 7 & 31.8 \\
\hline Fresh cut Christmas trees & 14 & 66.7 & 7 & 33.3 \\
\hline Painted poinsettias & 8 & 53.3 & 7 & 46.7 \\
\hline Artificial Christmas trees & 6 & 37.5 & 10 & 62.5 \\
\hline Interior Christmas decorating services & 5 & 35.7 & 9 & 64.3 \\
\hline Exterior Christmas decorating services & 3 & 21.4 & 11 & 78.6 \\
\hline Fresh cut flowers for Valentine's Day & 5 & 15.6 & 27 & 84.4 \\
\hline
\end{tabular}

Children's Activities. Ten respondents (71.4\%) will let children plant their own plants to take home. Four respondents (33.3\%) will use a petting zoo in the future. Three respondents $(27.3 \%)$ will use a corn maze in the future. Photos with the Easter Bunny 
will be used by three respondents $(23.1 \%)$. Two respondents $(16.7 \%)$ will create a haunted greenhouse in the future. Photos with Santa will be used by two respondents (15.4\%). Pony rides will be used in four garden centers $(12.5 \%)$. One respondent $(8.3 \%)$ will build and decorate a straw house. Eleven respondents $(100.0 \%)$ said they would not sell Easter pets (e. g. chicks, ducks, bunnies) in the future (see Table 40).

Table 40

Practices Garden Centers Will Use in the Future-Children's Activities

\begin{tabular}{|c|c|c|c|c|}
\hline & \multicolumn{2}{|c|}{ Yes } & \multicolumn{2}{|c|}{ No } \\
\hline & $N$ & $\%$ & $N$ & $\%$ \\
\hline Plant their own plants to take home & 10 & 71.4 & 4 & 28.6 \\
\hline Petting zoo & 4 & 33.3 & 8 & 66.7 \\
\hline Corn maze & 3 & 27.3 & 8 & 72.7 \\
\hline Photos with Easter Bunny & 3 & 23.1 & 10 & 76.9 \\
\hline Haunted greenhouse & 2 & 16.7 & 10 & 83.3 \\
\hline Photos with Santa & 2 & 15.4 & 11 & 84.6 \\
\hline Pony rides & 4 & 12.5 & 28 & 87.5 \\
\hline Build and decorate $d$ straw house & 1 & 8.3 & 11 & 91.7 \\
\hline Sell Easter pets (e. g. chicks, ducks, bunnies) & 0 & 0.0 & 11 & 100.0 \\
\hline
\end{tabular}

Advertisement. Twenty-three respondents (100.0\%) will use a company website. Fourteen respondents $(87.5 \%)$ will send periodic newsletters to customers. Twelve respondents $(85.7 \%)$ will email customers new product updates. Twenty respondents (76.9\%) will use the newspaper. The radio will be used by 17 respondents $(73.9 \%)$. Twelve respondents $(70.6 \%)$ will use a year round marketing program instead of a 
seasonal program. Fourteen respondents (45.2\%) will use television in the future (see Table 41).

Table 41

Practices Garden Centers Will Use in the Future-Advertisement

\begin{tabular}{lcccc}
\hline & \multicolumn{2}{c}{ Yes } & \multicolumn{2}{c}{ No } \\
\cline { 2 - 5 } & $N$ & $\%$ & $N$ & $\%$ \\
\hline Utilizing a company website & 23 & 100.0 & 0 & 0.0 \\
Sending periodic newsletters to customers & 14 & 87.5 & 2 & 12.5 \\
Emailing customers new product updates & 12 & 85.7 & 2 & 14.3 \\
Newspaper & 20 & 76.9 & 6 & 23.1 \\
Radio & 17 & 73.9 & 6 & 26.1 \\
Using a year round marketing program instead of seasonal & 12 & 70.6 & 5 & 29.4 \\
Television & 14 & 45.2 & 17 & 54.8 \\
\hline
\end{tabular}

Educational Seminars. Plants that attract butterflies will be used by 13 respondents $(81.2 \%)$. Fourteen respondents $(77.8 \%)$ will use gardening basics as a seminar topic. Plants that attract birds will be used as a seminar by 12 respondents (75.0\%). Ten respondents (71.4\%) will use dividing perennials as a seminar topic.

Eleven garden centers (64.7\%) will use cooking with herbs as a seminar. Pruning will be used by 10 respondents $(62.5 \%)$. Nine respondents $(60.0 \%)$ will use the dos and don'ts of much. Plants that repel pests (e. g. deer, rabbits, etc) will be used by seven respondents (53.8\%). Seven respondents (53.8\%) will use flower arranging as a seminar topic. 
Eight respondents $(53.3 \%)$ will use the seminar preparing gardens for winter. Six respondents $(50.0 \%)$ will use identifying diseases. Six garden centers (42.9\%) will use drying and storing herbs. Planting will be used as a seminar topic by 12 respondents (40.0\%). Identifying insect pests will be used by four respondents $(36.4 \%)$.

Drying flowers will be used by five garden centers (35.7\%). Four respondents $(33.3 \%)$ will use herbal health remedies. Using landscape lighting to accent gardens at night will be used by two respondents (18.2\%). Gardening without pain will be used by one respondent $(10.0 \%)$. One respondent $(9.1 \%)$ will use preserving birdhouses and feeders. Eleven respondents (100.0\%) will not use maintaining deck wood as a seminar. Twelve respondents (100.0\%) will not use maintaining outdoor furniture as a seminar (see Table 42).

Table 42

Practices Garden Centers Will Use in the Future-Educational Seminars

\begin{tabular}{lcccc}
\hline & \multicolumn{3}{c}{ Yes } & \multicolumn{2}{c}{ No } \\
\cline { 2 - 5 } & $N$ & $\%$ & $N$ & $\%$ \\
\hline Plants that attract butterflies & 13 & 81.2 & 3 & 18.8 \\
Gardening basics & 14 & 77.8 & 4 & 22.2 \\
Plants that attract birds & 12 & 75.0 & 4 & 25.0 \\
Dividing perennials & 10 & 71.4 & 4 & 28.6 \\
Cooking with herbs & 11 & 64.7 & 6 & 35.3 \\
Pruning & 10 & 62.5 & 6 & 37.5 \\
Dos and don'ts of mulch & 9 & 60.0 & 6 & 40.0 \\
Plants that repel pests (e. g. deer, rabbits, etc) & 7 & 53.8 & 6 & 46.2 \\
\hline
\end{tabular}


Table 42 (Continued)

Practices Garden Centers Will Use in the Future-Educational Seminars

\begin{tabular}{|c|c|c|c|c|}
\hline & \multicolumn{2}{|c|}{ Yes } & \multicolumn{2}{|c|}{ No } \\
\hline & $N$ & $\%$ & $N$ & $\%$ \\
\hline Flower arranging & 7 & 53.8 & 6 & 46.2 \\
\hline Preparing gardens for winter & 8 & 53.3 & 7 & 46.7 \\
\hline Identifying diseases & 6 & 50.0 & 6 & 50.0 \\
\hline Drying and storing herbs & 6 & 42.9 & 8 & 57.1 \\
\hline Planting & 12 & 40.0 & 18 & 60.0 \\
\hline Identifying insect pests & 4 & 36.4 & 7 & 63.6 \\
\hline Drying flowers & 5 & 35.7 & 9 & 64.3 \\
\hline Herbal health remedies & 4 & 33.3 & 8 & 66.7 \\
\hline Using landscape lighting to accent gardens at night & 2 & 18.2 & 9 & 81.8 \\
\hline Gardening without pain & 1 & 10.0 & 9 & 90.0 \\
\hline Preserving birdhouses and feeders & 1 & 9.1 & 10 & 90.9 \\
\hline Maintaining deck wood & 0 & 0.0 & 11 & 100.0 \\
\hline Maintaining outdoor furniture & 0 & 0.0 & 12 & 100.0 \\
\hline
\end{tabular}

Services for the Community. Twenty-one respondents $(65.6 \%)$ will donate supplies (e. g. mulch) to public areas. Twenty respondents $(90.0 \%)$ will donate plants to public areas. Four respondents $(40.0 \%)$ will sponsor a local craft show in the future. Four respondents (36.4\%) will offer a farmers market with free space for vendors. One 
respondent (11.1\%) will offer a farmers market with paid space for vendors as a service for the community (see Table 43).

Table 43

Practices Garden Centers Will Use in the Future-Services for the Community

\begin{tabular}{lcccr}
\hline & \multicolumn{2}{c}{ Yes } & No \\
\cline { 2 - 5 } & $N$ & $\%$ & $N$ & $\%$ \\
\hline Donation of supplies to public areas (e. g. mulch) & 21 & 65.6 & 11 & 34.4 \\
Donation of plants to public areas & 20 & 90.9 & 2 & 9.1 \\
Local craft show & 4 & 40.0 & 6 & 60.0 \\
Farmers market with free space for vendors & 4 & 36.4 & 7 & 63.6 \\
Farmers market with paid space for vendors & 1 & 11.1 & 8 & 88.9 \\
\hline
\end{tabular}

Services for Customers. Custom container design (e. g. hanging baskets, patio planters) will be used by 22 respondents $(91.7 \%)$. Seventeen respondents $(85.0 \%)$ will use customer designed containers (greenhouse grows until pick up date). Landscape design will be used by 18 respondents (75.0\%). Sixteen respondents $(72.7 \%)$ will use landscape installation.

Thirteen garden centers $(65.0 \%)$ will use pruning shrubs as a service for customers. Rental plants for weddings will be used by nine garden centers $(52.9 \%)$. Water garden design will be used by nine respondents (52.9\%). Pruning trees will be used by six respondents (37.5\%).

Five respondents $(35.7 \%)$ will use water garden installation. Four respondents (28.6\%) will use floral design for weddings (e. g. corsages). Rental plants for offices will 
be used by one respondent (8.3\%). Lawn equipment repair will be used by two garden centers $(6.2 \%)$ (see Table 44$)$.

Table 44

Practices Garden Centers Will Use in the Future-Services for Customers

\begin{tabular}{|c|c|c|c|c|}
\hline & \multicolumn{2}{|c|}{ Yes } & \multicolumn{2}{|c|}{ No } \\
\hline & $N$ & $\%$ & $N$ & $\%$ \\
\hline $\begin{array}{l}\text { Custom container design (e. g. hanging baskets, patio } \\
\text { planters) }\end{array}$ & 22 & 91.7 & 2 & 8.3 \\
\hline $\begin{array}{l}\text { Customer designed containers - greenhouse grows until } \\
\text { pickup date }\end{array}$ & 17 & 85.0 & 3 & 15.0 \\
\hline Landscape design & 18 & 75.0 & 6 & 25.0 \\
\hline Landscape installation & 16 & 72.7 & 6 & 27.3 \\
\hline Pruning shrubs & 13 & 65.0 & 7 & 35.0 \\
\hline Rental plants for weddings & 9 & 52.9 & 8 & 47.1 \\
\hline Water garden design & 9 & 52.9 & 8 & 47.1 \\
\hline Pruning trees & 6 & 37.5 & 10 & 62.5 \\
\hline Water garden installation & 5 & 35.7 & 9 & 64.3 \\
\hline Floral design for weddings (e. g. corsages) & 4 & 28.6 & 10 & 71.4 \\
\hline Rental plants for offices & 1 & 8.3 & 11 & 91.7 \\
\hline Lawn equipment repair & 2 & 6.2 & 30 & 93.8 \\
\hline
\end{tabular}

Lines of Plant Material. Perennials will be sold at 27 garden centers (96.4\%) in the future. Summer blooming perennials will be sold at 26 garden centers $(96.3 \%)$. Summer annuals (e. g. wave petunias) will be used in 25 garden centers (96.2\%). Twenty-three garden centers $(95.8 \%)$ will use outdoor container gardens. 
Cool weather annuals (e. g. pansies) will be used in 23 garden centers (95.8\%). Twenty-one respondents (91.3\%) will use larger sizes of annuals. Windowsill herb pots will be used by 18 garden centers $(90.0 \%)$. Twenty-four respondents $(88.9 \%)$ will use fall blooming ornamental grasses. Larger sizes of perennials will be used by 21 garden centers $(84.0 \%)$. Seventeen respondents $(81.0 \%)$ will sell house plants (e. g. African violets, ivy, etc). Fourteen respondents (70.0\%) will be using fall bulbs (e. g. tulips). Eleven garden centers (68.8\%) will use herb topiaries. Spring bulbs (e. g. dahlias) will be used by 10 respondents $(58.8 \%)$. Tropical bulbs (e. g. caladium) will be used by 10 respondents $(58.8 \%)$. Plant propagation supplies (e. g. seed potatoes, onion sets and garden seeds) will be sold in 17 garden centers (53.1\%). Ten respondents $(50.0 \%)$ will sell orchids in the future. Container evergreens for indoors will be used by eight garden centers $(50.0 \%)$. Fresh cut flowers year round will be sold at five garden centers $(41.7 \%)$ (see Table 45). 
Table 45

Practices Garden Centers Will Use in the Future-Lines of Plant Material

\begin{tabular}{|c|c|c|c|c|}
\hline & & & & \\
\hline & $N$ & $\%$ & $N$ & $\%$ \\
\hline Perennials & 27 & 96.4 & 1 & 3.6 \\
\hline Summer blooming perennials & 26 & 96.3 & 1 & 3.7 \\
\hline Summer annuals (e. g. wave petunias) & 25 & 96.2 & 1 & 3.8 \\
\hline Outdoor container gardens & 23 & 95.8 & 1 & 4.2 \\
\hline Cool weather annuals (e. g. pansies) & 23 & 95.8 & 1 & 4.2 \\
\hline Larger sizes of annuals & 21 & 91.3 & 2 & 8.7 \\
\hline Windowsill herb pots & 18 & 90.0 & 2 & 10.0 \\
\hline Fall blooming ornamental grasses & 24 & 88.9 & 3 & 11.1 \\
\hline Larger sizes of perennials & 21 & 84.0 & 4 & 16.0 \\
\hline House plants (e. g. African violets, ivy, etc) & 17 & 81.0 & 4 & 19.0 \\
\hline Fall bulbs (e. g. tulips) & 14 & 70.0 & 6 & 30.0 \\
\hline Herb topiaries & 11 & 68.8 & 5 & 31.2 \\
\hline Spring bulbs (e. g. dahlias) & 10 & 58.8 & 7 & 41.2 \\
\hline Tropical bulbs (e. g. caladium) & 10 & 58.8 & 7 & 41.2 \\
\hline $\begin{array}{l}\text { Plant propagation supplies (e. g. seed potatoes, onion } \\
\text { sets and garden seeds) }\end{array}$ & 17 & 53.1 & 15 & 46.9 \\
\hline Orchids & 10 & 50.0 & 10 & 50.0 \\
\hline Container evergreens for indoors & 8 & 50.0 & 8 & 50.0 \\
\hline Fresh cut flowers year round & 5 & 41.7 & 7 & 58.3 \\
\hline
\end{tabular}


Lines of Merchandise. Twenty-one respondents (91.3\%) will sell hard goods (e. g. pottery, statues, etc). Hummingbird supplies will be sold at 16 garden centers (76.2\%). Wind chimes will be sold in 15 garden centers $(75.0 \%)$. Outdoor furniture and accessories will be sold by 12 garden centers (66.7\%). Garden flags will be sold in 11 garden centers $(64.7 \%)$. Ten garden centers $(62.5 \%)$ will use candles and accessories.

Wildlife/bird feeding supplies will be sold at 12 garden centers (57.1\%). Three respondents (23.1\%) will sell garden themed clothing. Two respondents (18.2\%) will sell local jams and jellies. Local honey will be sold at two garden centers (16.7\%). Two garden centers (15.4\%) will sell pet food and supplies. Lawn equipment (e. g. mowers, weed trimmers) will be sold by two respondents (14.3\%). One garden center $(9.1 \%)$ will sell canning supplies. One garden center (8.3\%) will use local wine. One garden center (7.7\%) will sell other wine. One garden center (7.7\%) will sell other honey. Other jams and jellies will be sold by one garden center (3.3\%) (see Table 46). 
Table 46

Practices Garden Centers Will Use in the Future-Lines of Merchandise

\begin{tabular}{|c|c|c|c|c|}
\hline & \multicolumn{2}{|c|}{ Yes } & \multicolumn{2}{|c|}{ No } \\
\hline & $N$ & $\%$ & $N$ & $\%$ \\
\hline Hard goods (e. g. pottery, statues, etc) & 21 & 91.3 & 2 & 8.7 \\
\hline Hummingbird supplies & 16 & 76.2 & 5 & 23.8 \\
\hline Wind chimes & 15 & 75.0 & 5 & 25.0 \\
\hline Outdoor furniture and accessories & 12 & 66.7 & 6 & 33.3 \\
\hline Garden flags & 11 & 64.7 & 6 & 35.3 \\
\hline Candles and accessories & 10 & 62.5 & 6 & 37.5 \\
\hline Wildlife/bird feeding supplies & 12 & 57.1 & 9 & 42.9 \\
\hline Garden themed clothing & 3 & 23.1 & 10 & 76.9 \\
\hline Local jams and jellies & 2 & 18.2 & 9 & 81.8 \\
\hline Local honey & 2 & 16.7 & 10 & 83.3 \\
\hline Pet food and supplies & 2 & 15.4 & 11 & 84.6 \\
\hline Lawn equipment (e. g. mowers, weed trimmers) & 2 & 14.3 & 12 & 85.7 \\
\hline Canning supplies & 1 & 9.1 & 10 & 90.9 \\
\hline Local wine & 1 & 8.3 & 11 & 91.7 \\
\hline Other wine & 1 & 7.7 & 12 & 92.3 \\
\hline Other honey & 1 & 7.7 & 12 & 92.3 \\
\hline Other jams and jellies & 1 & 3.3 & 29 & 96.7 \\
\hline
\end{tabular}


Adjustments to Business or Location. Twenty-seven respondents (100.0\%) will hire knowledgeable and experienced staff. Twenty-six garden centers (100.0\%) will keep neat and clean aisles and displays. Customer friendly staff will be used by 26 respondents (96.3\%). Nineteen garden centers (82.6\%) will establish a warranty for plant material. Nineteen garden centers $(82.6 \%)$ will use display gardens. Twenty respondents $(80.0 \%)$ will grow their own plants to allow for diverse product mix. Fifteen respondents (78.9\%) will raise prices on all items. Thirteen garden centers (65.0\%) will add a covered shopping area for year round sales. Twelve respondents (63.2\%) will establish a "greeter" at the store entrance. A certified horticulturist will be hired at 10 garden centers (62.5\%). Three respondents (25.0\%) will add a coffee house in the future.

Seven garden centers (24.1\%) will become bonded. Two respondents (18.2\%) will add a bakery to the garden center. Two garden centers (18.2\%) will add an ice cream shop. Two garden centers (15.4\%) will convert a greenhouse to a banquet hall during non-peak months (see Table 47). 
Table 47

Practices Garden Centers Will Use in the Future-Adjustments to Business or Location

\begin{tabular}{|c|c|c|c|c|}
\hline & \multicolumn{2}{|c|}{ Yes } & \multicolumn{2}{|c|}{ No } \\
\hline & $N$ & $\%$ & $N$ & $\%$ \\
\hline Knowledgeable and experienced staff & 27 & 100.0 & 0 & 0.0 \\
\hline Neat and clean aisles and displays & 26 & 100.0 & 0 & 0.0 \\
\hline Customer friendly staff & 26 & 96.3 & 1 & 3.7 \\
\hline Establish warranty for plant material & 19 & 82.6 & 4 & 17.4 \\
\hline Display gardens & 19 & 82.6 & 4 & 17.4 \\
\hline $\begin{array}{l}\text { Growing your own plants to allow for diverse product } \\
\text { mix }\end{array}$ & 20 & 80.0 & 5 & 20.0 \\
\hline Raise prices on all items & 15 & 78.9 & 4 & 21.1 \\
\hline Add a covered shopping area for year round sales & 13 & 65.0 & 7 & 35.0 \\
\hline Establish a "greeter" at entrance & 12 & 63.2 & 7 & 36.8 \\
\hline Hire a certified horticulturist & 10 & 62.5 & 6 & 37.5 \\
\hline Add a coffee house & 3 & 25.0 & 9 & 75.0 \\
\hline Become bonded & 7 & 24.1 & 22 & 75.9 \\
\hline Add a bakery & 2 & 18.2 & 9 & 81.8 \\
\hline Add an ice cream shop & 2 & 18.2 & 9 & 81.8 \\
\hline $\begin{array}{l}\text { Convert greenhouse to banquet hall during non-peak } \\
\text { months }\end{array}$ & 2 & 15.4 & 11 & 84.6 \\
\hline
\end{tabular}


Customer Appreciation. Percent-off sale events will be used by 19 garden centers $(100.0 \%)$. Sixteen garden centers $(84.2 \%)$ will use seasonally dated coupons. Holiday specials on corresponding plants and accessories (i.e. Memorial Day: red, white and blue) will be offered by eight garden centers (57.1\%). Eight garden centers (57.1\%) will offer a spend $\$ 50$ get a free gift event.

Seven garden centers $(46.7 \%)$ will sell gift certificates at 20 percent off during non peak months. Four garden centers (36.4\%) will offer specials to customers in costume on Halloween. Five garden centers (35.7\%) will offer extra stamps on loyalty cards during non-peak months (see Table 48).

Table 48

Practices Garden Centers Will Use in the Future-Customer Appreciation

\begin{tabular}{lcccc}
\hline & \multicolumn{2}{l}{ Yes } & \multicolumn{2}{l}{ No } \\
\cline { 2 - 6 } & $N$ & $\%$ & $N$ & $\%$ \\
\hline Percent-off sale events & 19 & 100.0 & 0 & 0.0 \\
Seasonally dated coupons & 16 & 84.2 & 3 & 15.8 \\
$\begin{array}{l}\text { Holiday specials on corresponding plants and accessories } \\
\text { i.e. Memorial Day: red, white and blue }\end{array}$ & 8 & 57.1 & 6 & 42.9 \\
Spend \$50 get free gift & 8 & 57.1 & 6 & 42.9 \\
$\begin{array}{l}\text { Sell gift certificates at 20 percent off during non-peak } \\
\text { months }\end{array}$ & & & & \\
Offer specials to customers in costume on Halloween & 4 & 46.7 & 8 & 53.3 \\
Offer extra stamps on loyalty cards in non-peak months & 5 & 35.7 & 9 & 64.3 \\
\hline
\end{tabular}




\section{CHAPTER V}

\section{Summary, Conclusions, and Recommendations}

\section{Purpose of the Study}

The purpose of this study was to identify the measures that independently owned retail garden centers subscribe to extend their profitable season beyond the spring and early summer. By identifying profitable practices used by garden centers in the United States, the retail horticulture industry will benefit from increased profits and more employment opportunities. The entire green industry will also benefit from the increase in retail sales. The demand for plants, planting mediums and other garden accessories will have to increase to keep up with the retail sector. The manufacturing and production of plants, mediums and accessories will create more jobs and more money in the economy.

\section{Objectives of the Study}

The objectives for the study are reflected in the following research questions:

1. What measures are currently being practiced by garden centers to extend the profitable season beyond the spring and early summer?

2. What companion products do garden centers sell to extend the profitable season?

3. Which techniques used to extend the profitable season are viewed by retailers to be most helpful in raising profit margins?

4. Which techniques used to extend the profitable season are viewed by retailers to be most helpful in returning customers to the garden center? 
5. Which techniques are retailers most likely to incorporate in their current operation?

6. Is shutting down the garden center for an extended period of time a realistic option?

\section{Summary of Findings}

The sample population for this study was 248 independent retail garden centers that were members of the Ohio Floriculture Association as of November 2006 who resided in West Virginia, Ohio, Maryland, Pennsylvania, Virginia and Kentucky. The majority of garden centers represented in this study had been in operation for 20 or more years while the age of most respondents was between 50-59 years.

Typically the respondents were male and either had a high school diploma or a bachelors degree. Most of the respondents were not certified horticulturists. The majority of the garden centers were open year round and had an average of 15,790.32 square feet indoors and 42,509.50 square feet outdoors. Typically garden centers were open for a total of nine hours Monday through Friday, nine hours on Saturdays and five to six hours on Sundays.

\section{Research Questions One}

The top three practices currently subscribed by garden centers to extend the profitable season were identified in each of the 10 questionnaire categories. Live pumpkins, Halloween decorations and custom made decorations (e. g. wreaths, swags) were the most used Holiday Merchandise or Services. In the Children's Activities category letting children plant their own plants to take home, photos with Santa and a 
petting zoo were the most popular. The newspaper, utilizing a company website and the radio were the most used forms of advertisement.

Gardening basics, planting and plants that attract birds and butterflies were used the most as educational seminars. The most widely used services for the community were donation of plants to public areas, donation of supplies to public areas (e. g. mulch) and a local craft show. The most widely used services for customers were custom container design (e. g. hanging baskets, patio planters), landscape design and landscape installation.

The most popular lines of plant material were summer blooming perennials, perennials and summer annuals (e. g. wave petunias). The most popular lines of merchandise were hard goods (e. g. pottery, statues, etc), hummingbird supplies and wind chimes. The top three adjustments to the business or location garden centers already had in effect were knowledgeable and experienced staff, customer friendly staff and neat and clean aisles and displays. The three most popular forms of customer appreciation were percent-off sale events, seasonally dated coupons and spend $\$ 50$ get free gift.

\section{Research Question Two}

The companion products sold by garden centers to help extend the profitable season are reflected in the answers for research question one. Any products other than plant material can be considered companion products.

\section{Research Question Three}

Respondents rated the practices in the survey on the successfulness in raising profit margins of the garden center. The practices were rated as follows. 
Holiday Merchandise or Services. Live pumpkins, container trees, balled and burlap trees, custom made decorations (e. g. wreaths, swags), Christmas gift plants (e. g. poinsettias) and painted poinsettias were identified as moderately successful in raising profit margins. Easter decorations, Thanksgiving decorations, Halloween decorations, Christmas decorations, Interior Christmas decorating services, fresh cut Christmas trees, artificial Christmas trees, Easter gift plants (e. g Easter lilies) and fresh cut flowers for Valentine's Day were identified as slightly successful. Retailers identified exterior Christmas decorating services as unsuccessful in raising profit margins.

Children's Activities. Corn maze and plant their own plants to take home were identified as slightly successful. Photos with Easter Bunny, photos with Santa, petting zoo, selling Easter pets (e. g. chicks, ducks, bunnies), haunted greenhouse, build and decorate a straw house and pony rides were identified as unsuccessful in raising profit margins.

Advertisement. All of the practices involving advertisement were identified as moderately successful in raising profit margins. The practices were: emailing customers new product updates, utilizing a company website, sending periodic newsletters to customers, using a year round marketing program instead of seasonal, newspaper, radio and television.

Educational Seminars. Flower arranging was identified as moderately successful in raising profit margins. Preparing gardens for winter, drying and storing herbs, cooking with herbs, herbal health remedies, gardening basics, dos and don'ts of mulch, plants that attract birds, plants that attract butterflies, plants that repel pests (e. g. deer, rabbits, etc), identifying diseases, diving perennials, pruning and planting were identified as slightly 
successful. Drying flowers, maintaining deck wood, maintaining outdoor furniture, preserving birdhouses and feeders, using landscape lighting to accent gardens at night, identifying insect pests and gardening without pain were identified as unsuccessful.

Services for the Community. Local craft show, donation to plants to public areas and donation of supplies to public areas (e. g. mulch) were identified as slightly successful. Farmers market with free space for vendors and farmers market with paid space for vendors were identified as unsuccessful in raising profit margins.

Services for Customers. Landscape design, landscape installation, custom container design (e. g. hanging baskets, patio planters) and customer designed containers (greenhouse grows until pickup date) were identified as successful. Pruning shrubs and pruning trees were identified as moderately successful. Water garden design, water garden installation, rental plants for weddings and floral design for weddings (e. g. corsages) were identified as slightly successful. Rental plants for offices and lawn equipment repair were identified as unsuccessful in raising profit margins for the garden center.

Lines of Plant Material. Summer annuals (e. g. wave petunias), larger sizes of annuals, outdoor container gardens, perennials, larger sizes of perennials and summer blooming perennials were identified as successful in raising profit margins. Cool weather annuals (e. g. pansies), windowsill herb pots, herb topiaries, container evergreens for indoors, fall blooming ornamental grasses, orchids, house plants (e. g. African violets, ivy, etc) and plant propagation supplies (e. g. seed potatoes, onion sets and garden seeds) were identified as moderately successful. Fresh cut flowers year round, fall bulbs (e. g. 
tulips), spring bulbs (e. g. dahlias) and tropical bulbs (e. g. caladium) were identified as slightly successful.

Lines of Merchandise. Hard goods (e. g. pottery, statues, etc), garden flags, wind chimes and hummingbird supplies were identified as moderately successful. Outdoor furniture and accessories, candles and accessories, garden themed clothing, wildlife/bird feeding supplies, pet food and supplies and lawn equipment (e. g. mowers, weed trimmers) were identified as slightly successful. Canning supplies, local wine, other wine, local honey, other honey, local jams and jellies and other jams and jellies were identified as unsuccessful in raising profit margins.

Adjustments to Business or Location. Display gardens, growing your own plants to allow for diverse product mix, neat and clean aisles and displays, knowledgeable and experienced staff, customer friendly staff, establish a "greeter" at entrance and raise prices on all items were identified as successful in raising profit margins. Add a covered shopping area for year round sales, establish warranty for plant material, hire a certified horticulturist and become bonded were identified as moderately successful. Add a coffee house was identified as slightly successful. Add a bakery, add an ice cream shop and convert greenhouse to banquet hall during non-peak months were identified as unsuccessful in raising profit margins.

Customer Appreciation. Seasonally dated coupons, percent-off sale events, sell gift certificates at 20 percent off during non-peak months, spend $\$ 50$ get free gift and holiday specials on corresponding plants and accessories (i.e. Memorial Day: red, white and blue) were identified as moderately successful. Offer extra stamps on loyalty cards 
in non-peak months was rated slightly successful. Offer specials to customers in costume on Halloween was identified as unsuccessful in raising profit margins.

\section{Research Question Four}

Respondents rated the practices in the survey on successfulness in returning customers to the store. The practices were rated as follows.

Holiday Merchandise or Services. Halloween decorations, live pumpkins, Christmas decorations, fresh cut Christmas trees, container trees, balled and burlap trees, custom made decorations (e. g. wreaths, swags), Christmas gift plants (e. g. poinsettias), painted poinsettias, Easter gift plants (e. g. Easter lilies) and fresh cut flowers for Valentine's Day were identified as moderately successful. Easter decorations, Thanksgiving decorations, Interior Christmas decorating services, Exterior Christmas decorating services and artificial Christmas trees were identified as slightly successful in returning customers to the store.

Children's Activities. Corn maze, petting zoo, plant their own plants to take home and pony rides were identified as moderately successful. Photos with Easter Bunny, photos with Santa and haunted greenhouse were identified as slightly successful. Sell Easter pets (e. g. chicks, ducks, bunnies) and build and decorate a straw house were identified as unsuccessful in returning customers to the store.

Advertisement. All practices involving advertisement were rated moderately successful in returning customers to the store. The practices were emailing customers new product updates, utilizing a company website, sending periodic newsletters to customers, using a year round marketing program instead of seasonal, newspaper, radio and television. 
Educational Seminars. Cooking with herbs, herbal health remedies, gardening basics, plants that attract butterflies, flower arranging, dividing perennials, pruning and planting were identified as moderately successful in returning customers to the store. Preparing gardens for winter, drying flowers, drying and storing herbs, dos and don'ts of mulch, plants that attract birds, plants that repel pests (e. g. deer, rabbits, etc), identifying insect pests, identifying diseases and gardening without pain were identified as slightly successful. Maintaining deck wood, maintaining outdoor furniture, preserving birdhouses and feeders and using landscape lighting to accent gardens at night were identified as unsuccessful.

Services for the Community. Local craft show, donation of plants to public areas and donation of supplies to public areas (e. g. mulch) were identified as slightly successful. Farmers market with free space for vendors and farmers market with paid space for vendors were identified as unsuccessful in returning customers to the store.

Services for Customers. Custom container design (e. g. hanging baskets, patio planters) and customer designed containers (greenhouse grows until pickup date) were identified as successful services for customers. Landscape design, landscape installation, pruning shrubs and pruning trees were identified as moderately successful. Water garden design, water garden installation, rental plants for weddings and floral design for weddings (e. g. corsages) were identified as slightly successful. Rental plants for offices and lawn equipment repair were identified as unsuccessful in returning customers to the store.

Lines of Plant Material. Cool weather annuals (e. g. pansies), summer annuals (e. g. wave petunias), larger sizes of annuals, outdoor container gardens, perennials, larger 
sizes of perennials and summer blooming perennials were identified as successful lines of plant material. Windowsill herb pots, herb topiaries, container evergreens for indoors, fall blooming ornamental grasses, orchids, fresh cut flowers year round, house plants (e. g. African violets, ivy, etc) and plant propagation supplies (e. g. seed potatoes, onion sets and garden seeds) were identified as moderately successful. Spring bulbs (e. g. dahlias) and tropical bulbs (e. g. caladium) were identified as slightly successful in returning customers to the store.

Lines of Merchandise. Hard goods (e. g. pottery, statues, etc) was identified as successful in returning customers to the store. Outdoor furniture and accessories, garden flags, wind chimes and hummingbird supplies were identified as moderately successful. Candles and accessories, garden themed clothing, wildlife/bird feeding supplies, pet food and supplies, lawn equipment (e. g. mowers, weed trimmers) and local honey were identified as slightly successful. Canning supplies, local wine, other wine, other honey, local jams and jellies and other jams and jellies were identified as unsuccessful in returning customers to the store.

Adjustments to Business or Location. Display gardens, growing your own plants to allow for diverse product mix, neat and clean aisles and displays, knowledgeable and experienced staff, customer friendly staff, establish a "greeter" at entrance and raise prices on all items were identified as successful adjustments. Add a covered shopping area for year round sales, establish warranty for plant material, hire a certified horticulturist and become bonded were identified as moderately successful. Add a coffee house, add a bakery, add an ice cream shop and convert greenhouse to banquet hall 
during non-peak months were identified as unsuccessful in returning customers to the store.

Customer Appreciation. Seasonally dated coupons, percent-off sale events, sell gift certificates at 20 percent off during non-peak months, spend $\$ 50$ get free gift, offer extra stamps on loyalty cards in non-peak months and holiday specials on corresponding plants and accessories (i.e. Memorial Day: red, white and blue) were identified as moderately successful in returning customers to the store. Offer specials to customers in costume on Halloween was identified as unsuccessful in returning customers to the store.

\section{Research Question Five}

The practices which garden centers are most likely to use in the future were identified in each of the questionnaire categories. In holiday merchandise and services; live pumpkins, Halloween decorations and custom made decorations (e. g. wreaths, swags) were most likely to be used later by retailers. Plant their own plants to take home, pony rides and petting zoo were most likely to be used in the future as children's activities. Utilizing a company website, newspaper and radio are the most popular forms of advertising retailers will use in the future.

Gardening basics, plants that attract butterflies and plants that attract birds were most likely to be used as seminar topics in the future. Donation of supplies to public areas (e. g. mulch), donation of plants to public areas and local craft show are most likely to be used as services for the community. Custom container design (e. g. hanging baskets, patio planters), landscape design and customer designed containers (greenhouse grows until pickup date) were most likely to be used as services for customers. 
Perennials, summer blooming perennials and summer annuals (e. g. wave petunias) were most likely to be added as lines of plant material. Hard goods (e. g. pottery, statues, etc), hummingbird supplies and wind chimes are most likely to be lines of merchandise carried at garden centers in the future. Knowledgeable and experienced staff, neat and clean aisles and displays and customer friendly staff were most likely to be implemented in the garden center. Percent-off sale events, seasonally dated coupons and spend $\$ 50$ get free gift were most likely to be customer appreciation events in the future.

\section{Research Question Six}

Sixty five percent of retailers agreed that shutting down the garden center for an extended period of time was not a realistic option. However, thirty five percent said that garden centers should only be open during the spring and summer.

\section{Conclusions}

Based on the results of this study, the following conclusions were made:

1. Over half of the practices in the study were rated moderately successful or higher in returning customers to the store.

2. Less than half of the practices in the study were rated moderately successful or higher in raising profit margins.

3. Customer friendly staff had the highest successfulness rating in both returning customers and raising profit margins as identified by independent garden center retailers.

4. The 10 most successful practices in getting customers to return identified by independent garden center retailers are (in order of successfulness); customer friendly staff, knowledgeable and experienced staff, growing your own plants to 
allow for diverse product mix, larger sizes of annuals, summer annuals (e. g. wave petunias), neat and clean aisles and displays, perennials, customer designed containers (greenhouse grows until pickup date), larger sizes of perennials and summer blooming perennials.

5. The 10 most successful practices in raising profit margins identified by independent garden center retailers are (in order of successfulness); customer friendly staff, knowledgeable and experienced staff, growing your own plants to allow for diverse product mix, raise prices on all items, larger sizes of annuals, custom container design (e. g. hanging baskets, patio planters), display gardens, establish a "greeter" at entrance, summer annuals (e. g. wave petunias) and customer designed containers (greenhouse grows until pickup date).

6. More than half of the respondents' garden centers are open year round.

7. Nearly two-thirds of independent garden center retailers think garden centers should be open for business year round.

8. Seven practices in the study were not being used by garden centers. These included: sell Easter pets (e. g. chicks, ducks, bunnies), maintaining deck wood, maintaining outdoor furniture, preserving birdhouses and feeders, using landscape lighting to accent gardens at night, add a bakery and add an ice cream shop.

9. All respondents in the study were using the following practices: knowledgeable and experienced staff and customer friendly staff.

10. Three practices in the study will not be used in the future by garden centers. These include: sell Easter pets (e. g. chicks, ducks, bunnies), maintaining deck wood and maintaining outdoor furniture. 
11. All respondents in the study will use the following practices in the future: utilizing a company website, neat and clean aisles and displays, knowledgeable and experienced staff and percent-off sale events.

12. Garden center retailers are willing to try new practices in order to raise profit margins or return customers to the store.

\section{Recommendations}

The researcher makes the following recommendations based on the results of the research:

1. This study should be replicated with additional independent retail garden centers across the country.

2. This study should be conducted at a better time for garden centers. Perhaps during non-peak months.

3. Independent retail garden centers should continue to look "outside the box" for ways to return customers to the store as well as raise profit margins.

4. Independent retail garden centers should acquire retail horticulture publications which are often full of ideas on returning customers to the store and raising profit margins.

5. Independent retail garden centers should implement the top 10 practices identified through this study as a foundation for returning customers to the store and raising profit margins. 


\section{REFERENCES}

Aker, S., Cromell, C., DiNella, G. R., Eames-Sheavly, M., Macunovich, J., Mellor, D., et al. (2005). Better homes and gardens: New garden book. Des Moines, Iowa: Meredith Corporation.

Ary, D., Jacobs, L. C., Razavieh, A., \& Sorensen, C. (2006). Introduction to research in education $\left(7^{\text {th }}\right.$ ed.). California: Thomson Wadsworth.

Behe, K., Pfahl, P. B., \& Hofmann, C. (1994). The retail florist business. Danville, Illinois: The Interstate Publishers, Inc.

Boyer, M. (2007). Getting to know mulch. Lawn \& Garden Retailer, 6, 58-59.

Calkins, B. (2006). Art plants. Green Profit, 10, 34.

Caras, D. \& Ferguson, S. (2004). Shedding light on bulbs. American Nurseryman, 200, 22-27.

Childress, M. (2006, December 25). In the business of merriment. Charleston Daily Mail, p. $2 \mathrm{D}$.

City of Morgantown. (2006). Zoning ordinance as amended July 18, 2006. Retrieved January 20, 2007, from http://www.morgantown.com/zoning-ordinance.htm.

Cohen, S. (2004). Summer school. American Nurseryman, 199, 22-27.

Dillman, D. A. (2000). Mail and internet surveys: The tailored design method ( $7^{\text {th }}$ ed.). New York: John Wiley \& Sons, Inc.

Fornari, C. L. (2005). Cultivating success. American Nurseryman, 202, 24-27.

Gilmer, M. (2006). Planting outside prime time. Lawn and Garden Retailer, 5, 18-21.

Hall, C. R., Hodges, A. W., Haydu, J. J. (2004). Economic impacts of the green industry in the United States. University of Tennessee: Institute of Agriculture. Retrieved January 20, 2007, from http://www.utextension.utk.edu/hbin/greenimpact.html.

Hamernik, H. (1997). On growing and marketing perennials. Retrieved September 17, 2006, from http://www.greenbeam.com/features/they062397.html.

Hsu, E. (2005). Winter scents. American Nurseryman, 202, 22-25.

Krejcie, R., \& Morgan, D. (1970). Determining sample size for research activities. Educational and Psychological Measurement, 30, 607-610. 
Landicho, S. (2003). Gardens of distinction. American Nurseryman, 197, 20-26.

Mish, F. C. (Ed.). (1997). The Merriam-Webster dictionary. Massachusetts: MerriamWebster, Inc.

National Weather Service Forecast Office. (2005, December). Spring/fall freeze maps. Retrieved October 2, 2006, from, http://www.erh.noaa.gov/rlx/climate/springfreeze.html \& http://www.erh.noaa.gov/rlx/climate/fallfreeze.html.

OFA: An Association of Floriculture Professionals. (2006). Mission statement. Retrieved November 12, 2006 from http://www.ofa.org/index2.html.

Robinson, J. P., Shaver, P. R., \& Wrightsman, L. S. (1991). Criteria for scale selection and evaluation. In J. P. Robinson, P. R. Shaver, \& L. S. Wrightsman (Eds.). Measures of personality and social psychological attitudes (pp. 1-16) New York: Academic Press.

Sharpton, J. (2005, July). Consumer trends and your garden center. Symposium conducted at the Ohio Floriculture Association Short Course, Columbus, Ohio.

Shoemaker, M. (2003). Stand by your fans. Green Profit, 7, 18.

Shoemaker, M. (2003). Retail from the crypt. Green Profit, 7, 15.

Speichert, G. (2005). The color of marketing. American Nurseryman, 202, 16-20.

Weishar, J. (2006). Changing customer habits. Lawn and Garden Retailer, 5, 12-16.

Williams, G. S. (1975). Greenhouse flowers and bedding plants for agribusiness studies. Danville, Illinois: The Interstate Printers \& Publishers, Inc. 


\section{APPENDICES}


APPENDIX A:

Phase I Questionnaire 


\section{Current Practices Utilized by Independent Garden Centers to Extend the Profitable Season by Returning Customers, Raising Profit Margins and Implementing New Practices}

A. What are the three best strategies, directly related to horticulture, that you use to extend the profitable season of your garden center?

1.

2.

3.

B. What are the three best strategies, NOT directly related to horticulture, that you use to extend the profitable season of your garden center?

1.

2.

3.

Thank you! Please return this form in the enclosed postage-paid, self-addressed envelope. We sincerely appreciate your cooperation.

If you have questions about the survey, please contact me at: nhunt@mix.wvu.edu or call (304) 692-5686. 
APPENDIX B:

Initial Post Card 


\section{Dear Garden Center Manager:}

In the next few days you will be receiving a questionnaire as part of a research study to determine the current practices utilized by independent retail garden centers to extend the profitable season. Your response may have an impact on the retail horticulture industry. The results of the study will be used to partially fulfill the requirements for a Master of Science Degree in Agricultural and Extension Education. I do hope you'll make the time to complete the survey when it arrives.

Thank you for your time!

Nona Kay Hunt

Agricultural and Extension Education

Davis College of Agriculture, Forestry, and Consumer Sciences

West Virginia University

2048 Agricultural Sciences Building

P.O. Box 6108

Morgantown, WV 26506-6108 
APPENDIX C:

Cover Letter 
March 20, 2007

Dear Garden Center Manager:

Retail horticulture has traditionally been a seasonal business. However, more and more garden centers are trying to make it a successful business year round. Extending the profitable season may assist in increasing your firm's yearly profit. Several publications are available as resources for ways to extend the profitable season. Research must be done to determine what methods are being used by garden centers to extend the season and if these methods are returning a profit for the company.

The purpose of this research study is to determine the practices being utilized by independent retail garden centers to extend the profitable season beyond the spring and early summer months. The results of this study will be used to prepare a thesis to partially fulfill the requirements for a Master of Science Degree in Agricultural and Extension Education. By determining what is currently being practiced to extend the profitable season, other garden centers might be able to improve their profit margins as well.

Participation in this research study is completely voluntary and all information you provide will be held as confidential as possible. Your response to the survey is critical to the success of the study and will only take a few minutes of your time to complete. A copy of the results of the study will be available upon request. You may skip any question you are not comfortable answering. You will notice a code number at the top left of the return envelope. This code will be used to identify non-respondents for follow-up purposes and will be destroyed before the data are analyzed. Survey results will be reported in a summary format and individual responses will not be identifiable.

Please place the completed questionnaire in the enclosed postage-paid selfaddressed return envelop and drop it in the mail. Please return your completed questionnaire before April 5, 2007. We thank you in advance for your assistance with this research effort. We sincerely appreciate your time and effort.

Sincerely,

Nona Kay Hunt

Graduate Student
Stacy A. Gartin

Professor \& Chair 
APPENDIX D:

Phase II Questionnaire 


\section{Current Practices Utilized by Independent Garden Centers to Extend the Profitable Season by Returning Customers, Raising Profit Margins and Implementing New Practices}

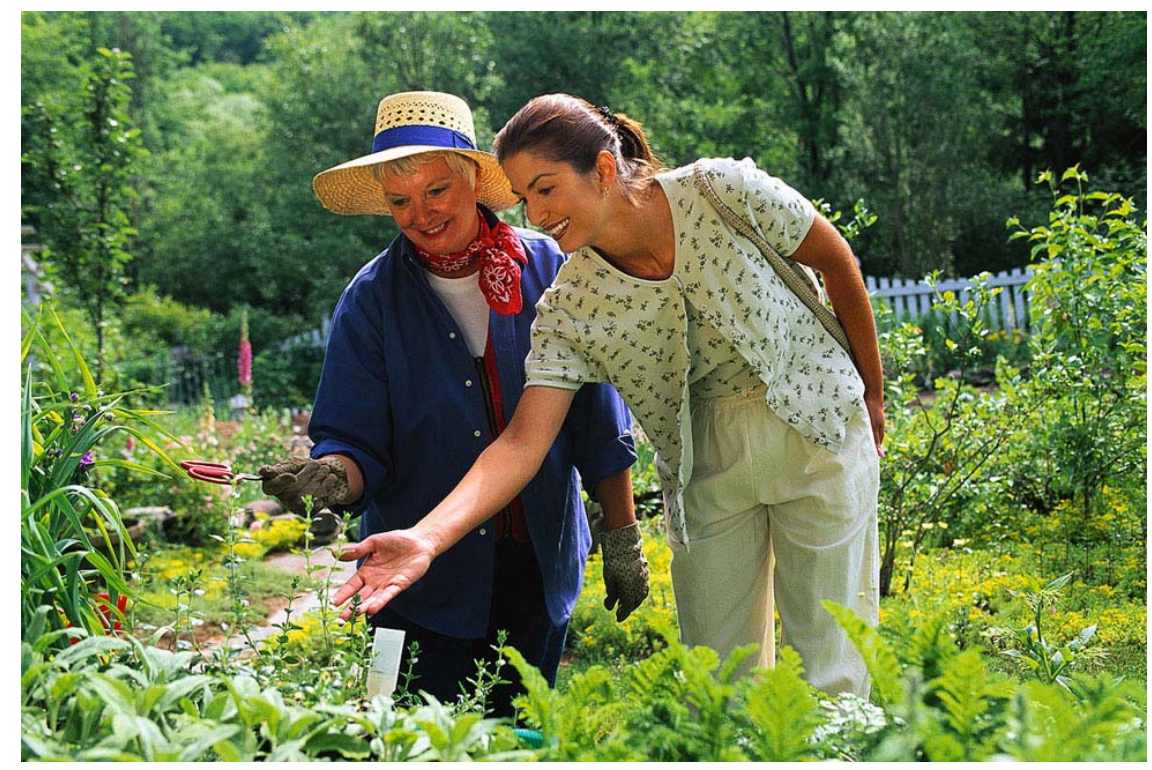

Nona Kay Hunt

Graduate Student

Agricultural and Extension Education

Davis College of Agriculture, Forestry and Consumer Sciences

West Virginia University

Morgantown, WV 26506 


\section{Current Practices Utilized by Independent Garden Centers to Extend the Profitable Season by Returning Customers, Raising Profit Margins and Implementing New Practices}

The purpose of this study is to determine successful practices being utilized by garden centers to extend the profitable season. The list of practices was compiled from popular retail horticulture publications.

Instructions: Please circle the appropriate response below to indicate your answer to the following questions. Are you currently using this practice? What is the potential for success of this practice in getting customers to return and raising profit margins? And will you use this practice in the future? After each category there is space for you to list other practices not mentioned in the survey. Please list any practices and rate them on the same scale.

\begin{tabular}{|c|c|c|c|c|c|c|c|c|c|c|c|c|}
\hline \multirow{3}{*}{ Practice } & \multirow{2}{*}{\multicolumn{2}{|c|}{$\begin{array}{c}\text { Are you } \\
\text { currently } \\
\text { using } \\
\text { this } \\
\text { practice? }\end{array}$}} & \multicolumn{8}{|c|}{ Potential for success in: } & \multirow{2}{*}{\multicolumn{2}{|c|}{$\begin{array}{l}\text { Will you } \\
\text { use this } \\
\text { practice } \\
\text { in the } \\
\text { future? }\end{array}$}} \\
\hline & & & \multicolumn{4}{|c|}{$\begin{array}{l}\text { Getting } \\
\text { customers to } \\
\text { return? }\end{array}$} & \multicolumn{4}{|c|}{$\begin{array}{l}\text { Raising profit } \\
\text { margins? }\end{array}$} & & \\
\hline & $\stackrel{\infty}{\infty}$ & $\stackrel{0}{z}$ & 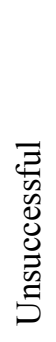 & 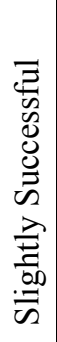 & 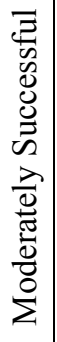 & 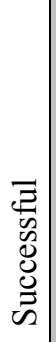 & 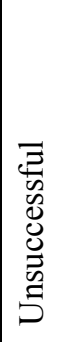 & 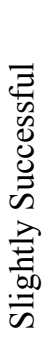 & 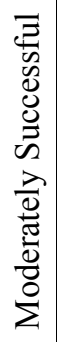 & $\begin{array}{l}\vec{Z} \\
\tilde{w} \\
0 \\
0 \\
0 \\
\tilde{n}\end{array}$ & $\stackrel{\infty}{\infty}$ & $\stackrel{\circ}{z}$ \\
\hline \multicolumn{13}{|c|}{ Do you offer the following holiday merchandise or services? } \\
\hline 1. Easter decorations & $\mathrm{Y}$ & $\mathrm{N}$ & 1 & 2 & 3 & 4 & 1 & 2 & 3 & 4 & Y & $\mathrm{N}$ \\
\hline 2. Thanksgiving decorations & Y & $\mathrm{N}$ & 1 & 2 & 3 & 4 & 1 & 2 & 3 & 4 & $\mathrm{Y}$ & $\mathrm{N}$ \\
\hline 3. Halloween decorations & $\mathrm{Y}$ & $\mathrm{N}$ & 1 & 2 & 3 & 4 & 1 & 2 & 3 & 4 & $\mathrm{Y}$ & $\mathrm{N}$ \\
\hline 4. Live pumpkins & $\mathrm{Y}$ & $\mathrm{N}$ & 1 & 2 & 3 & 4 & 1 & 2 & 3 & 4 & $\mathrm{Y}$ & $\mathrm{N}$ \\
\hline 5. Christmas decorations & $\mathrm{Y}$ & $\mathrm{N}$ & 1 & 2 & 3 & 4 & 1 & 2 & 3 & 4 & $\mathrm{Y}$ & $\mathrm{N}$ \\
\hline $\begin{array}{l}\text { 6. Interior Christmas decorating } \\
\text { services }\end{array}$ & $\mathrm{Y}$ & $\mathrm{N}$ & 1 & 2 & 3 & 4 & 1 & 2 & 3 & 4 & $\mathrm{Y}$ & $\mathrm{N}$ \\
\hline $\begin{array}{l}\text { 7. Exterior Christmas decorating } \\
\text { services }\end{array}$ & $\mathrm{Y}$ & $\mathrm{N}$ & 1 & 2 & 3 & 4 & 1 & 2 & 3 & 4 & $\mathrm{Y}$ & $\mathrm{N}$ \\
\hline 8. Fresh cut Christmas trees & $\mathrm{Y}$ & $\mathrm{N}$ & 1 & 2 & 3 & 4 & 1 & 2 & 3 & 4 & $\mathrm{Y}$ & $\mathrm{N}$ \\
\hline 9. Container trees & Y & $\mathrm{N}$ & 1 & 2 & 3 & 4 & 1 & 2 & 3 & 4 & $\mathrm{Y}$ & $\mathrm{N}$ \\
\hline 10. Balled and burlap trees & $\mathrm{Y}$ & $\mathrm{N}$ & 1 & 2 & 3 & 4 & 1 & 2 & 3 & 4 & $\mathrm{Y}$ & $\mathrm{N}$ \\
\hline 11. Artificial Christmas trees & $\mathrm{Y}$ & $\mathrm{N}$ & 1 & 2 & 3 & 4 & 1 & 2 & 3 & 4 & $\mathrm{Y}$ & $\mathrm{N}$ \\
\hline
\end{tabular}




\begin{tabular}{|c|c|c|c|c|c|c|c|c|c|c|c|c|}
\hline \multirow{3}{*}{ Practice } & \multirow{2}{*}{\multicolumn{2}{|c|}{$\begin{array}{l}\text { Are you } \\
\text { currently } \\
\text { using } \\
\text { this } \\
\text { practice? }\end{array}$}} & \multicolumn{8}{|c|}{ Potential for success in: } & \multirow{2}{*}{\multicolumn{2}{|c|}{$\begin{array}{l}\text { Will you } \\
\text { use this } \\
\text { practice } \\
\text { in the } \\
\text { future? }\end{array}$}} \\
\hline & & & \multicolumn{4}{|c|}{$\begin{array}{l}\text { Getting } \\
\text { customers to } \\
\text { return? }\end{array}$} & \multicolumn{4}{|c|}{$\begin{array}{l}\text { Raising profit } \\
\text { margins? }\end{array}$} & & \\
\hline & $\stackrel{\infty}{\infty}$ & z & 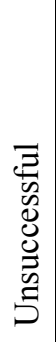 & 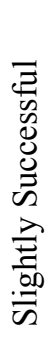 & 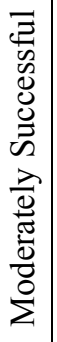 & 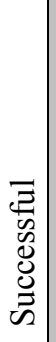 & 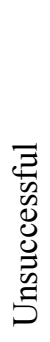 & 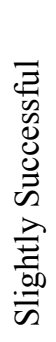 & 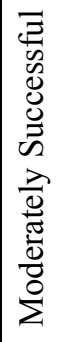 & 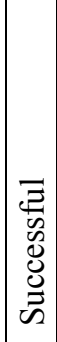 & $\stackrel{0}{\nu}$ & $\stackrel{\circ}{z}$ \\
\hline \multicolumn{13}{|c|}{ Do you offer the following holiday merchandise or services? (continued) } \\
\hline $\begin{array}{l}\text { 12. Custom made decorations } \\
\text { (e.g. wreaths, swags) }\end{array}$ & $\mathrm{Y}$ & $\mathrm{N}$ & 1 & 2 & 3 & 4 & 1 & 2 & 3 & 4 & $\mathrm{Y}$ & $\mathrm{N}$ \\
\hline $\begin{array}{l}\text { 13. Christmas gift plants (e.g. } \\
\text { poinsettias) }\end{array}$ & $\mathrm{Y}$ & $\mathrm{N}$ & 1 & 2 & 3 & 4 & 1 & 2 & 3 & 4 & $\mathrm{Y}$ & $\mathrm{N}$ \\
\hline 14. Painted poinsettias & $\mathrm{Y}$ & $\mathrm{N}$ & 1 & 2 & 3 & 4 & 1 & 2 & 3 & 4 & $\mathrm{Y}$ & $\mathrm{N}$ \\
\hline $\begin{array}{l}\text { 15. Easter gift plants (e.g. Easter } \\
\text { lilies) }\end{array}$ & $\mathrm{Y}$ & $\mathrm{N}$ & 1 & 2 & 3 & 4 & 1 & 2 & 3 & 4 & $\mathrm{Y}$ & $\mathrm{N}$ \\
\hline $\begin{array}{l}\text { 16. Fresh cut flowers for } \\
\text { Valentine's Day }\end{array}$ & $\mathrm{Y}$ & $\mathrm{N}$ & 1 & 2 & 3 & 4 & 1 & 2 & 3 & 4 & $\mathrm{Y}$ & $\mathrm{N}$ \\
\hline 17. & $\mathrm{Y}$ & $\mathrm{N}$ & 1 & 2 & 3 & 4 & 1 & 2 & 3 & 4 & $\mathrm{Y}$ & $\mathrm{N}$ \\
\hline 18. & $\mathrm{Y}$ & $\mathrm{N}$ & 1 & 2 & 3 & 4 & 1 & 2 & 3 & 4 & $\mathrm{Y}$ & $\mathrm{N}$ \\
\hline \multicolumn{13}{|c|}{ Do you offer the following children's activities? } \\
\hline 19. Photos with Easter Bunny & $\mathrm{Y}$ & $\mathrm{N}$ & 1 & 2 & 3 & 4 & 1 & 2 & 3 & 4 & $\mathrm{Y}$ & $\mathrm{N}$ \\
\hline 20. Photos with Santa & $\mathrm{Y}$ & $\mathrm{N}$ & 1 & 2 & 3 & 4 & 1 & 2 & 3 & 4 & $\mathrm{Y}$ & $\mathrm{N}$ \\
\hline 21. Corn maze & $\mathrm{Y}$ & $\mathrm{N}$ & 1 & 2 & 3 & 4 & 1 & 2 & 3 & 4 & $\mathrm{Y}$ & $\mathrm{N}$ \\
\hline 22. Petting zoo & $\mathrm{Y}$ & $\mathrm{N}$ & 1 & 2 & 3 & 4 & 1 & 2 & 3 & 4 & $\mathrm{Y}$ & $\mathrm{N}$ \\
\hline $\begin{array}{l}\text { 23. Sell Easter pets (e.g. chicks, } \\
\text { ducks, bunnies) }\end{array}$ & $\mathrm{Y}$ & $\mathrm{N}$ & 1 & 2 & 3 & 4 & 1 & 2 & 3 & 4 & $\mathrm{Y}$ & $\mathrm{N}$ \\
\hline $\begin{array}{l}\text { 24. Plant their own plants to take } \\
\text { home }\end{array}$ & $\mathrm{Y}$ & $\mathrm{N}$ & 1 & 2 & 3 & 4 & 1 & 2 & 3 & 4 & $\mathrm{Y}$ & $\mathrm{N}$ \\
\hline 25. Haunted greenhouse & $\mathrm{Y}$ & $\mathrm{N}$ & 1 & 2 & 3 & 4 & 1 & 2 & 3 & 4 & $\mathrm{Y}$ & $\mathrm{N}$ \\
\hline $\begin{array}{l}\text { 26. Build and decorate a straw } \\
\text { house }\end{array}$ & $\mathrm{Y}$ & $\mathrm{N}$ & 1 & 2 & 3 & 4 & 1 & 2 & 3 & 4 & $\mathrm{Y}$ & $\mathrm{N}$ \\
\hline 27. Pony rides & $\mathrm{Y}$ & $\mathrm{N}$ & 1 & 2 & 3 & 4 & 1 & 2 & 3 & 4 & $\mathrm{Y}$ & $\mathrm{N}$ \\
\hline 28. & $\mathrm{Y}$ & $\mathrm{N}$ & 1 & 2 & 3 & 4 & 1 & 2 & 3 & 4 & $\mathrm{Y}$ & $\mathrm{N}$ \\
\hline 29. & $\mathrm{Y}$ & $\mathrm{N}$ & 1 & 2 & 3 & 4 & 1 & 2 & 3 & 4 & $\mathrm{Y}$ & $\mathrm{N}$ \\
\hline
\end{tabular}




\begin{tabular}{|c|c|c|c|c|c|c|c|c|c|c|c|c|}
\hline \multirow{3}{*}{ Practice } & \multirow{2}{*}{\multicolumn{2}{|c|}{$\begin{array}{l}\text { Are you } \\
\text { currently } \\
\text { using } \\
\text { this } \\
\text { practice? }\end{array}$}} & \multicolumn{8}{|c|}{ Potential for success in: } & \multirow{2}{*}{\multicolumn{2}{|c|}{$\begin{array}{l}\text { Will you } \\
\text { use this } \\
\text { practice } \\
\text { in the } \\
\text { future? }\end{array}$}} \\
\hline & & & \multicolumn{4}{|c|}{$\begin{array}{l}\text { Getting } \\
\text { customers to } \\
\text { return? }\end{array}$} & \multicolumn{4}{|c|}{$\begin{array}{l}\text { Raising profit } \\
\text { margins? }\end{array}$} & & \\
\hline & $\stackrel{\theta}{\nu}$ & 之 & 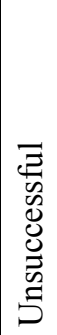 & 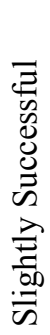 & 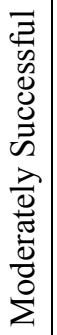 & 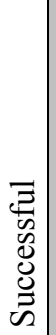 & 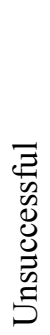 & 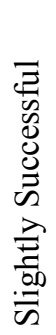 & 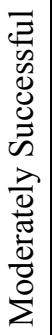 & 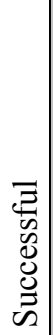 & $\stackrel{\infty}{\nu}$ & z \\
\hline \multicolumn{13}{|l|}{ Do you advertise by: } \\
\hline $\begin{array}{l}\text { 30. Emailing customers new } \\
\text { product updates }\end{array}$ & $\mathrm{Y}$ & $\mathrm{N}$ & 1 & 2 & 3 & 4 & 1 & 2 & 3 & 4 & $\mathrm{Y}$ & $\mathrm{N}$ \\
\hline 31. Utilizing a company website & $\mathrm{Y}$ & $\mathrm{N}$ & 1 & 2 & 3 & 4 & 1 & 2 & 3 & 4 & $\mathrm{Y}$ & $\mathrm{N}$ \\
\hline $\begin{array}{l}\text { 32. Sending periodic newsletters } \\
\text { to customers }\end{array}$ & $\mathrm{Y}$ & $\mathrm{N}$ & 1 & 2 & 3 & 4 & 1 & 2 & 3 & 4 & $\mathrm{Y}$ & $\mathrm{N}$ \\
\hline $\begin{array}{l}\text { 33. Using a year round marketing } \\
\text { program instead of seasonal }\end{array}$ & $\mathrm{Y}$ & $\mathrm{N}$ & 1 & 2 & 3 & 4 & 1 & 2 & 3 & 4 & $\mathrm{Y}$ & $\mathrm{N}$ \\
\hline 34. Newspaper & $\mathrm{Y}$ & $\mathrm{N}$ & 1 & 2 & 3 & 4 & 1 & 2 & 3 & 4 & $\mathrm{Y}$ & $\mathrm{N}$ \\
\hline 35. Radio & $\mathrm{Y}$ & $\mathrm{N}$ & 1 & 2 & 3 & 4 & 1 & 2 & 3 & 4 & $\mathrm{Y}$ & $\mathrm{N}$ \\
\hline 36. Television & $\mathrm{Y}$ & $\mathrm{N}$ & 1 & 2 & 3 & 4 & 1 & 2 & 3 & 4 & $\mathrm{Y}$ & $\mathrm{N}$ \\
\hline 37. & $\mathrm{Y}$ & $\mathrm{N}$ & 1 & 2 & 3 & 4 & 1 & 2 & 3 & 4 & $\mathrm{Y}$ & $\mathrm{N}$ \\
\hline 38. & $\mathrm{Y}$ & $\mathrm{N}$ & 1 & 2 & 3 & 4 & 1 & 2 & 3 & 4 & $\mathrm{Y}$ & $\mathrm{N}$ \\
\hline \multicolumn{13}{|c|}{ Do you provide educational seminars for customers on the following topics? } \\
\hline 39. Preparing gardens for winter & $\mathrm{Y}$ & $\mathrm{N}$ & 1 & 2 & 3 & 4 & 1 & 2 & 3 & 4 & $\mathrm{Y}$ & $\mathrm{N}$ \\
\hline 40. Drying flowers & $\mathrm{Y}$ & $\mathrm{N}$ & 1 & 2 & 3 & 4 & 1 & 2 & 3 & 4 & $\mathrm{Y}$ & $\mathrm{N}$ \\
\hline 41. Drying and storing herbs & $\mathrm{Y}$ & $\mathrm{N}$ & 1 & 2 & 3 & 4 & 1 & 2 & 3 & 4 & $\mathrm{Y}$ & $\mathrm{N}$ \\
\hline 42. Cooking with herbs & $\mathrm{Y}$ & $\mathrm{N}$ & 1 & 2 & 3 & 4 & 1 & 2 & 3 & 4 & $\mathrm{Y}$ & $\mathrm{N}$ \\
\hline 43. Herbal health remedies & $\mathrm{Y}$ & $\mathrm{N}$ & 1 & 2 & 3 & 4 & 1 & 2 & 3 & 4 & $\mathrm{Y}$ & $\mathrm{N}$ \\
\hline 44. Maintaining deck wood & $\mathrm{Y}$ & $\mathrm{N}$ & 1 & 2 & 3 & 4 & 1 & 2 & 3 & 4 & $\mathrm{Y}$ & $\mathrm{N}$ \\
\hline 45. Maintaining outdoor furniture & $\mathrm{Y}$ & $\mathrm{N}$ & 1 & 2 & 3 & 4 & 1 & 2 & 3 & 4 & $\mathrm{Y}$ & $\mathrm{N}$ \\
\hline $\begin{array}{l}\text { 46. Preserving birdhouses and } \\
\text { feeders }\end{array}$ & $\mathrm{Y}$ & $\mathrm{N}$ & 1 & 2 & 3 & 4 & 1 & 2 & 3 & 4 & $\mathrm{Y}$ & $\mathrm{N}$ \\
\hline $\begin{array}{l}\text { 47. Using landscape lighting to } \\
\text { accent gardens at night }\end{array}$ & $\mathrm{Y}$ & $\mathrm{N}$ & 1 & 2 & 3 & 4 & 1 & 2 & 3 & 4 & $\mathrm{Y}$ & $\mathrm{N}$ \\
\hline 48. Gardening basics & $\mathrm{Y}$ & $\mathrm{N}$ & 1 & 2 & 3 & 4 & 1 & 2 & 3 & 4 & $\mathrm{Y}$ & $\mathrm{N}$ \\
\hline 49. Dos and don'ts of mulch & $\mathrm{Y}$ & $\mathrm{N}$ & 1 & 2 & 3 & 4 & 1 & 2 & 3 & 4 & $\mathrm{Y}$ & $\mathrm{N}$ \\
\hline
\end{tabular}




\begin{tabular}{|c|c|c|c|c|c|c|c|c|c|c|c|c|}
\hline \multirow{3}{*}{ Practice } & \multirow{2}{*}{\multicolumn{2}{|c|}{$\begin{array}{l}\text { Are you } \\
\text { currently } \\
\text { using } \\
\text { this } \\
\text { practice? }\end{array}$}} & \multicolumn{8}{|c|}{ Potential for success in: } & \multirow{2}{*}{\multicolumn{2}{|c|}{$\begin{array}{l}\text { Will you } \\
\text { use this } \\
\text { practice } \\
\text { in the } \\
\text { future? }\end{array}$}} \\
\hline & & & \multicolumn{4}{|c|}{$\begin{array}{l}\text { Getting } \\
\text { customers to } \\
\text { return? }\end{array}$} & \multicolumn{4}{|c|}{$\begin{array}{l}\text { Raising profit } \\
\text { margins? }\end{array}$} & & \\
\hline & $\stackrel{0}{\nu}$ & $\stackrel{\circ}{Z}$ & 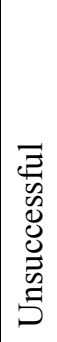 & 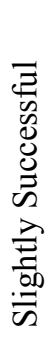 & 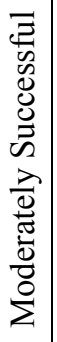 & $\begin{array}{l}\vec{Z} \\
\tilde{D} \\
0 \\
0 \\
0 \\
\tilde{n}\end{array}$ & 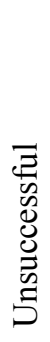 & 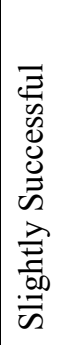 & 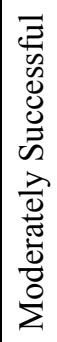 & 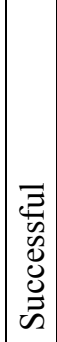 & $\sum_{i}^{\infty}$ & ż \\
\hline \multicolumn{13}{|c|}{ Do you provide educational seminars for customers on the following topics? (continued) } \\
\hline 50. Plants that attract birds & $\mathrm{Y}$ & $\mathrm{N}$ & 1 & 2 & 3 & 4 & 1 & 2 & 3 & 4 & $\mathrm{Y}$ & $\mathrm{N}$ \\
\hline 51. Plants that attract butterflies & $\mathrm{Y}$ & $\mathrm{N}$ & 1 & 2 & 3 & 4 & 1 & 2 & 3 & 4 & $\mathrm{Y}$ & $\mathrm{N}$ \\
\hline $\begin{array}{l}\text { 52. Plants that repel pests (e.g. } \\
\text { deer, rabbits, etc.) }\end{array}$ & $\mathrm{Y}$ & $\mathrm{N}$ & 1 & 2 & 3 & 4 & 1 & 2 & 3 & 4 & $\mathrm{Y}$ & $\mathrm{N}$ \\
\hline 53. Identifying insect pests & $\mathrm{Y}$ & $\mathrm{N}$ & 1 & 2 & 3 & 4 & 1 & 2 & 3 & 4 & $\mathrm{Y}$ & $\mathrm{N}$ \\
\hline 54. Identifying diseases & $\mathrm{Y}$ & $\mathrm{N}$ & 1 & 2 & 3 & 4 & 1 & 2 & 3 & 4 & $\mathrm{Y}$ & $\mathrm{N}$ \\
\hline 55. Gardening without pain & $\mathrm{Y}$ & $\mathrm{N}$ & 1 & 2 & 3 & 4 & 1 & 2 & 3 & 4 & $\mathrm{Y}$ & $\mathrm{N}$ \\
\hline 56. Flower arranging & $\mathrm{Y}$ & $\mathrm{N}$ & 1 & 2 & 3 & 4 & 1 & 2 & 3 & 4 & $\mathrm{Y}$ & $\mathrm{N}$ \\
\hline 57. Dividing perennials & $\mathrm{Y}$ & $\mathrm{N}$ & 1 & 2 & 3 & 4 & 1 & 2 & 3 & 4 & $\mathrm{Y}$ & $\mathrm{N}$ \\
\hline 58. Pruning & $\mathrm{Y}$ & $\mathrm{N}$ & 1 & 2 & 3 & 4 & 1 & 2 & 3 & 4 & $\mathrm{Y}$ & $\mathrm{N}$ \\
\hline 59. Planting & $\mathrm{Y}$ & $\mathrm{N}$ & 1 & 2 & 3 & 4 & 1 & 2 & 3 & 4 & $\mathrm{Y}$ & $\mathrm{N}$ \\
\hline 60. & $\mathrm{Y}$ & $\mathrm{N}$ & 1 & 2 & 3 & 4 & 1 & 2 & 3 & 4 & $\mathrm{Y}$ & $\mathrm{N}$ \\
\hline 61. & $\mathrm{Y}$ & $\mathrm{N}$ & 1 & 2 & 3 & 4 & 1 & 2 & 3 & 4 & $\mathrm{Y}$ & $\mathrm{N}$ \\
\hline \multicolumn{13}{|c|}{ Do you offer the following services for the community? } \\
\hline 62. Local craft show & $\mathrm{Y}$ & $\mathrm{N}$ & 1 & 2 & 3 & 4 & 1 & 2 & 3 & 4 & $\mathrm{Y}$ & $\mathrm{N}$ \\
\hline $\begin{array}{l}\text { 63. Farmers market with free } \\
\text { space for vendors }\end{array}$ & Y & $\mathrm{N}$ & 1 & 2 & 3 & 4 & 1 & 2 & 3 & 4 & $\mathrm{Y}$ & $\mathrm{N}$ \\
\hline $\begin{array}{l}\text { 64. Farmers market with paid } \\
\text { space for vendors }\end{array}$ & $\mathrm{Y}$ & $\mathrm{N}$ & 1 & 2 & 3 & 4 & 1 & 2 & 3 & 4 & $\mathrm{Y}$ & $\mathrm{N}$ \\
\hline $\begin{array}{l}\text { 65. Donation of plants to public } \\
\text { areas }\end{array}$ & $\mathrm{Y}$ & $\mathrm{N}$ & 1 & 2 & 3 & 4 & 1 & 2 & 3 & 4 & $\mathrm{Y}$ & $\mathrm{N}$ \\
\hline $\begin{array}{l}\text { 66. Donation of supplies to public } \\
\text { areas (e.g. mulch) }\end{array}$ & $\mathrm{Y}$ & $\mathrm{N}$ & 1 & 2 & 3 & 4 & 1 & 2 & 3 & 4 & $\mathrm{Y}$ & $\mathrm{N}$ \\
\hline \multicolumn{13}{|c|}{ Do you offer the following services for customers? } \\
\hline 67. Landscape design & $\mathrm{Y}$ & $\mathrm{N}$ & 1 & 2 & 3 & 4 & 1 & 2 & 3 & 4 & $\mathrm{Y}$ & $\mathrm{N}$ \\
\hline 68. Landscape installation & $\mathrm{Y}$ & $\mathrm{N}$ & 1 & 2 & 3 & 4 & 1 & 2 & 3 & 4 & $\mathrm{Y}$ & $\mathrm{N}$ \\
\hline
\end{tabular}




\begin{tabular}{|c|c|c|c|c|c|c|c|c|c|c|c|c|}
\hline \multirow{3}{*}{ Practice } & \multirow{2}{*}{\multicolumn{2}{|c|}{$\begin{array}{l}\text { Are you } \\
\text { currently } \\
\text { using } \\
\text { this } \\
\text { practice? }\end{array}$}} & \multicolumn{8}{|c|}{ Potential for success in: } & \multirow{2}{*}{\multicolumn{2}{|c|}{$\begin{array}{l}\text { Will you } \\
\text { use this } \\
\text { practice } \\
\text { in the } \\
\text { future? }\end{array}$}} \\
\hline & & & \multicolumn{4}{|c|}{$\begin{array}{l}\text { Getting } \\
\text { customers to } \\
\text { return? }\end{array}$} & \multicolumn{4}{|c|}{$\begin{array}{l}\text { Raising profit } \\
\text { margins? }\end{array}$} & & \\
\hline & $\stackrel{\theta}{\nu}$ & z & 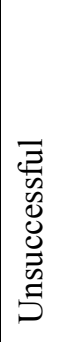 & 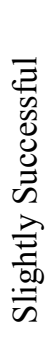 & 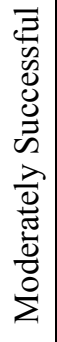 & 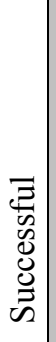 & 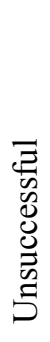 & 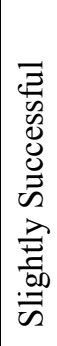 & 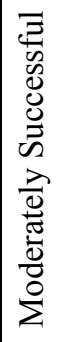 & 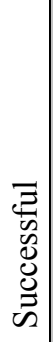 & $\stackrel{0}{\nu}$ & z \\
\hline \multicolumn{13}{|c|}{ Do you offer the following services for customers? (continued) } \\
\hline 69. Water garden design & $\mathrm{Y}$ & $\mathrm{N}$ & 1 & 2 & 3 & 4 & 1 & 2 & 3 & 4 & $\mathrm{Y}$ & $\mathrm{N}$ \\
\hline 70. Water garden installation & $\mathrm{Y}$ & $\mathrm{N}$ & 1 & 2 & 3 & 4 & 1 & 2 & 3 & 4 & $\mathrm{Y}$ & $\mathrm{N}$ \\
\hline $\begin{array}{l}\text { 71. Custom container design (e.g. } \\
\text { hanging baskets, patio } \\
\text { planters) }\end{array}$ & $\mathrm{Y}$ & $\mathrm{N}$ & 1 & 2 & 3 & 4 & 1 & 2 & 3 & 4 & $\mathrm{Y}$ & $\mathrm{N}$ \\
\hline $\begin{array}{l}\text { 72. Customer designed containers } \\
\text { - greenhouse grows until } \\
\text { pickup date }\end{array}$ & $\mathrm{Y}$ & $\mathrm{N}$ & 1 & 2 & 3 & 4 & 1 & 2 & 3 & 4 & $\mathrm{Y}$ & $\mathrm{N}$ \\
\hline 73. Pruning shrubs & $\mathrm{Y}$ & $\mathrm{N}$ & 1 & 2 & 3 & 4 & 1 & 2 & 3 & 4 & $\mathrm{Y}$ & $\mathrm{N}$ \\
\hline 74. Pruning trees & $\mathrm{Y}$ & $\mathrm{N}$ & 1 & 2 & 3 & 4 & 1 & 2 & 3 & 4 & $\mathrm{Y}$ & $\mathrm{N}$ \\
\hline 75. Rental plants for weddings & $\mathrm{Y}$ & $\mathrm{N}$ & 1 & 2 & 3 & 4 & 1 & 2 & 3 & 4 & $\mathrm{Y}$ & $\mathrm{N}$ \\
\hline 76. Rental plants for offices & $\mathrm{Y}$ & $\mathrm{N}$ & 1 & 2 & 3 & 4 & 1 & 2 & 3 & 4 & $\mathrm{Y}$ & $\mathrm{N}$ \\
\hline $\begin{array}{l}\text { 77. Floral design for weddings } \\
\text { (e.g. corsages) }\end{array}$ & $\mathrm{Y}$ & $\mathrm{N}$ & 1 & 2 & 3 & 4 & 1 & 2 & 3 & 4 & $\mathrm{Y}$ & $\mathrm{N}$ \\
\hline 78. Lawn equipment repair & $\mathrm{Y}$ & $\mathrm{N}$ & 1 & 2 & 3 & 4 & 1 & 2 & 3 & 4 & Y & $\mathrm{N}$ \\
\hline 79. & $\mathrm{Y}$ & $\mathrm{N}$ & 1 & 2 & 3 & 4 & 1 & 2 & 3 & 4 & $\mathrm{Y}$ & $\mathrm{N}$ \\
\hline 80. & $\mathrm{Y}$ & $\mathrm{N}$ & 1 & 2 & 3 & 4 & 1 & 2 & 3 & 4 & $\mathrm{Y}$ & $\mathrm{N}$ \\
\hline \multicolumn{13}{|c|}{ Do you offer the following lines of plant material? } \\
\hline $\begin{array}{l}\text { 81. Cool weather annuals (e.g. } \\
\text { pansies) }\end{array}$ & $\mathrm{Y}$ & $\mathrm{N}$ & 1 & 2 & 3 & 4 & 1 & 2 & 3 & 4 & $\mathrm{Y}$ & $\mathrm{N}$ \\
\hline $\begin{array}{l}\text { 82. Summer annuals (e.g. wave } \\
\text { petunias) }\end{array}$ & $\mathrm{Y}$ & $\mathrm{N}$ & 1 & 2 & 3 & 4 & 1 & 2 & 3 & 4 & $\mathrm{Y}$ & $\mathrm{N}$ \\
\hline 83. Larger sizes of annuals & $\mathrm{Y}$ & $\mathrm{N}$ & 1 & 2 & 3 & 4 & 1 & 2 & 3 & 4 & $\mathrm{Y}$ & $\mathrm{N}$ \\
\hline 84. Windowsill herb pots & $\mathrm{Y}$ & $\mathrm{N}$ & 1 & 2 & 3 & 4 & 1 & 2 & 3 & 4 & $\mathrm{Y}$ & $\mathrm{N}$ \\
\hline 85. Herb topiaries & $\mathrm{Y}$ & $\mathrm{N}$ & 1 & 2 & 3 & 4 & 1 & 2 & 3 & 4 & $\mathrm{Y}$ & $\mathrm{N}$ \\
\hline 86. Outdoor container gardens & $\mathrm{Y}$ & $\mathrm{N}$ & 1 & 2 & 3 & 4 & 1 & 2 & 3 & 4 & $\mathrm{Y}$ & $\mathrm{N}$ \\
\hline $\begin{array}{l}\text { 87. Container evergreens for } \\
\text { indoors }\end{array}$ & $\mathrm{Y}$ & $\mathrm{N}$ & 1 & 2 & 3 & 4 & 1 & 2 & 3 & 4 & $\mathrm{Y}$ & $\mathrm{N}$ \\
\hline
\end{tabular}




\begin{tabular}{|c|c|c|c|c|c|c|c|c|c|c|c|c|}
\hline \multirow{3}{*}{ Practice } & \multirow{2}{*}{\multicolumn{2}{|c|}{$\begin{array}{l}\text { Are you } \\
\text { currently } \\
\text { using } \\
\text { this } \\
\text { practice? }\end{array}$}} & \multicolumn{8}{|c|}{ Potential for success in: } & \multirow{2}{*}{\multicolumn{2}{|c|}{$\begin{array}{l}\text { Will you } \\
\text { use this } \\
\text { practice } \\
\text { in the } \\
\text { future? }\end{array}$}} \\
\hline & & & \multicolumn{4}{|c|}{$\begin{array}{l}\text { Getting } \\
\text { customers to } \\
\text { return? }\end{array}$} & \multicolumn{4}{|c|}{$\begin{array}{l}\text { Raising profit } \\
\text { margins? }\end{array}$} & & \\
\hline & $\stackrel{\infty}{\infty}$ & 之 & 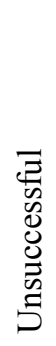 & 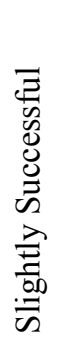 & 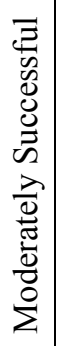 & 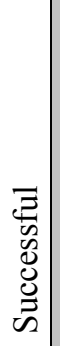 & 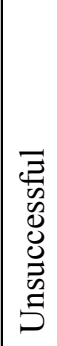 & 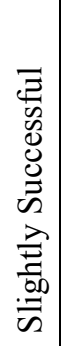 & 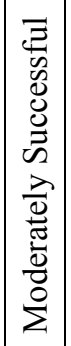 & 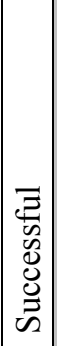 & $\stackrel{\infty}{\infty}$ & z \\
\hline \multicolumn{13}{|c|}{ Do you offer the following lines of plant material? (continued) } \\
\hline $\begin{array}{l}\text { 88. Fall blooming ornamental } \\
\text { grasses }\end{array}$ & $\mathrm{Y}$ & $\mathrm{N}$ & 1 & 2 & 3 & 4 & 1 & 2 & 3 & 4 & $\mathrm{Y}$ & $\mathrm{N}$ \\
\hline 89. Perennials & $\mathrm{Y}$ & $\mathrm{N}$ & 1 & 2 & 3 & 4 & 1 & 2 & 3 & 4 & $\bar{Y}$ & $\mathrm{~N}$ \\
\hline 90. Larger sizes of perennials & $\mathrm{Y}$ & $\mathrm{N}$ & 1 & 2 & 3 & 4 & 1 & 2 & 3 & 4 & $\mathrm{Y}$ & $\mathrm{N}$ \\
\hline 91. Orchids & Y & $\mathrm{N}$ & 1 & 2 & 3 & 4 & 1 & 2 & 3 & 4 & $\mathrm{Y}$ & $\mathrm{N}$ \\
\hline 92. Fresh cut flowers year round & $\mathrm{Y}$ & $\mathrm{N}$ & 1 & 2 & 3 & 4 & 1 & 2 & 3 & 4 & $\mathrm{Y}$ & $\mathrm{N}$ \\
\hline 93. Summer blooming perennials & Y & $\mathrm{N}$ & 1 & 2 & 3 & 4 & 1 & 2 & 3 & 4 & $\mathrm{Y}$ & $\mathrm{N}$ \\
\hline 94. Fall bulbs (e.g. tulips) & $\mathrm{Y}$ & $\mathrm{N}$ & 1 & 2 & 3 & 4 & 1 & 2 & 3 & 4 & $\mathrm{Y}$ & $\mathrm{N}$ \\
\hline 95. Spring bulbs (e.g. dahlias) & $\mathrm{Y}$ & $\mathrm{N}$ & 1 & 2 & 3 & 4 & 1 & 2 & 3 & 4 & $\mathrm{Y}$ & $\mathrm{N}$ \\
\hline 96. Tropical bulbs (e.g. caladium) & $\mathrm{Y}$ & $\mathrm{N}$ & 1 & 2 & 3 & 4 & 1 & 2 & 3 & 4 & $\mathrm{Y}$ & $\mathrm{N}$ \\
\hline $\begin{array}{l}\text { 97. House plants (e.g. African } \\
\text { violets, ivy, etc.) }\end{array}$ & $\mathrm{Y}$ & $\mathrm{N}$ & 1 & 2 & 3 & 4 & 1 & 2 & 3 & 4 & $\mathrm{Y}$ & $\mathrm{N}$ \\
\hline $\begin{array}{l}\text { 98. Plant propagation supplies } \\
\text { (e.g. seed potatoes, onion sets } \\
\text { and garden seeds) }\end{array}$ & $\mathrm{Y}$ & $\mathrm{N}$ & 1 & 2 & 3 & 4 & 1 & 2 & 3 & 4 & $\mathrm{Y}$ & $\mathrm{N}$ \\
\hline 99. & $\mathrm{Y}$ & $\mathrm{N}$ & 1 & 2 & 3 & 4 & 1 & 2 & 3 & 4 & $\mathrm{Y}$ & $\mathrm{N}$ \\
\hline 100. & $\mathrm{Y}$ & $\mathrm{N}$ & 1 & 2 & 3 & 4 & 1 & 2 & 3 & 4 & $\mathrm{Y}$ & $\mathrm{N}$ \\
\hline \multicolumn{13}{|c|}{ Do you offer the following lines of merchandise? } \\
\hline $\begin{array}{l}\text { 101. Outdoor furniture and } \\
\text { accessories }\end{array}$ & $\mathrm{Y}$ & $\mathrm{N}$ & 1 & 2 & 3 & 4 & 1 & 2 & 3 & 4 & $\mathrm{Y}$ & $\mathrm{N}$ \\
\hline $\begin{array}{l}\text { 102. Hard goods (e.g. pottery, } \\
\text { statues, etc.) }\end{array}$ & $\mathrm{Y}$ & $\mathrm{N}$ & 1 & 2 & 3 & 4 & 1 & 2 & 3 & 4 & $\mathrm{Y}$ & $\mathrm{N}$ \\
\hline 103. Garden flags & $\mathrm{Y}$ & $\mathrm{N}$ & 1 & 2 & 3 & 4 & 1 & 2 & 3 & 4 & $\mathrm{Y}$ & $\mathrm{N}$ \\
\hline 104. Wind chimes & Y & $\mathrm{N}$ & 1 & 2 & 3 & 4 & 1 & 2 & 3 & 4 & $\mathrm{Y}$ & $\mathrm{N}$ \\
\hline 105. Canning supplies & $\mathrm{Y}$ & $\mathrm{N}$ & 1 & 2 & 3 & 4 & 1 & 2 & 3 & 4 & $\mathrm{Y}$ & $\mathrm{N}$ \\
\hline 106. Candles and accessories & $\mathrm{Y}$ & $\mathrm{N}$ & 1 & 2 & 3 & 4 & 1 & 2 & 3 & 4 & $\mathrm{Y}$ & $\mathrm{N}$ \\
\hline 107. Garden themed clothing & $\mathrm{Y}$ & $\mathrm{N}$ & 1 & 2 & 3 & 4 & 1 & 2 & 3 & 4 & $\mathrm{Y}$ & $\mathrm{N}$ \\
\hline 108. Wildlife/bird feeding supplies & $\mathrm{Y}$ & $\mathrm{N}$ & 1 & 2 & 3 & 4 & 1 & 2 & 3 & 4 & $\mathrm{Y}$ & $\mathrm{N}$ \\
\hline
\end{tabular}




\begin{tabular}{|c|c|c|c|c|c|c|c|c|c|c|c|c|}
\hline \multirow{3}{*}{ Practice } & \multirow{2}{*}{\multicolumn{2}{|c|}{$\begin{array}{c}\text { Are you } \\
\text { currently } \\
\text { using } \\
\text { this } \\
\text { practice? }\end{array}$}} & \multicolumn{8}{|c|}{ Potential for success in: } & \multirow{2}{*}{\multicolumn{2}{|c|}{$\begin{array}{l}\text { Will you } \\
\text { use this } \\
\text { practice } \\
\text { in the } \\
\text { future? }\end{array}$}} \\
\hline & & & \multicolumn{4}{|c|}{$\begin{array}{l}\text { Getting } \\
\text { customers to } \\
\text { return? }\end{array}$} & \multicolumn{4}{|c|}{$\begin{array}{l}\text { Raising profit } \\
\text { margins? }\end{array}$} & & \\
\hline & $\stackrel{\infty}{\infty}$ & 之 & 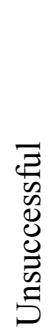 & 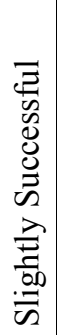 & 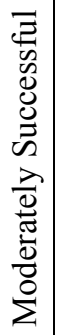 & $\begin{array}{c} \\
\\
ت \\
0 \\
0 \\
0 \\
0 \\
心 \\
心\end{array}$ & 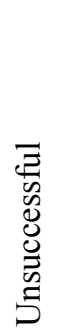 & 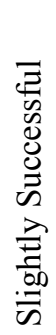 & 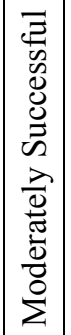 & 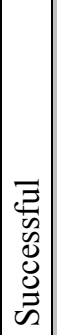 & $\stackrel{\infty}{\infty}$ & $\stackrel{\circ}{z}$ \\
\hline \multicolumn{13}{|c|}{ Do you offer the following lines of merchandise? (continued) } \\
\hline 109. Hummingbird supplies & $\mathrm{Y}$ & $\mathrm{N}$ & 1 & 2 & 3 & 4 & 1 & 2 & 3 & 4 & $\mathrm{Y}$ & $\mathrm{N}$ \\
\hline 110. Pet food and supplies & $\mathrm{Y}$ & $\mathrm{N}$ & 1 & 2 & 3 & 4 & 1 & 2 & 3 & 4 & $\mathrm{Y}$ & $\mathrm{N}$ \\
\hline $\begin{array}{l}\text { 111. Lawn equipment (e.g. } \\
\text { mowers, weed trimmers) }\end{array}$ & $\mathrm{Y}$ & $\mathrm{N}$ & 1 & 2 & 3 & 4 & 1 & 2 & 3 & 4 & $\mathrm{Y}$ & $\mathrm{N}$ \\
\hline 112. Local wine & $\mathrm{Y}$ & $\mathrm{N}$ & 1 & 2 & 3 & 4 & 1 & 2 & 3 & 4 & $\mathrm{Y}$ & $\mathrm{N}$ \\
\hline 113. Other wine & $\mathrm{Y}$ & $\mathrm{N}$ & 1 & 2 & 3 & 4 & 1 & 2 & 3 & 4 & $\mathrm{Y}$ & $\mathrm{N}$ \\
\hline 114. Local honey & $\mathrm{Y}$ & $\mathrm{N}$ & 1 & 2 & 3 & 4 & 1 & 2 & 3 & 4 & $\mathrm{Y}$ & $\mathrm{N}$ \\
\hline 115. Other honey & $\mathrm{Y}$ & $\mathrm{N}$ & 1 & 2 & 3 & 4 & 1 & 2 & 3 & 4 & $\mathrm{Y}$ & $\mathrm{N}$ \\
\hline 116. Local jams and jellies & $\mathrm{Y}$ & $\mathrm{N}$ & 1 & 2 & 3 & 4 & 1 & 2 & 3 & 4 & $\mathrm{Y}$ & $\mathrm{N}$ \\
\hline 117. Other jams and jellies & $\mathrm{Y}$ & $\mathrm{N}$ & 1 & 2 & 3 & 4 & 1 & 2 & 3 & 4 & $\mathrm{Y}$ & $\mathrm{N}$ \\
\hline 118. & $\mathrm{Y}$ & $\mathrm{N}$ & 1 & 2 & 3 & 4 & 1 & 2 & 3 & 4 & $\mathrm{Y}$ & $\mathrm{N}$ \\
\hline 119. & $\mathrm{Y}$ & $\mathrm{N}$ & 1 & 2 & 3 & 4 & 1 & 2 & 3 & 4 & $\mathrm{Y}$ & $\mathrm{N}$ \\
\hline \multicolumn{13}{|c|}{ Have you made the following adjustments to the business and/or location? } \\
\hline $\begin{array}{l}\text { 120. Add a covered shopping area } \\
\text { for year round sales }\end{array}$ & $\mathrm{Y}$ & $\mathrm{N}$ & 1 & 2 & 3 & 4 & 1 & 2 & 3 & 4 & $\mathrm{Y}$ & $\mathrm{N}$ \\
\hline 121. Display gardens & $\mathrm{Y}$ & $\mathrm{N}$ & 1 & 2 & 3 & 4 & 1 & 2 & 3 & 4 & $\mathrm{Y}$ & $\mathrm{N}$ \\
\hline $\begin{array}{l}\text { 122. Growing your own plants to } \\
\text { allow for diverse product mix }\end{array}$ & $\mathrm{Y}$ & $\mathrm{N}$ & 1 & 2 & 3 & 4 & 1 & 2 & 3 & 4 & $\mathrm{Y}$ & $\mathrm{N}$ \\
\hline $\begin{array}{l}\text { 123. Neat and clean aisles and } \\
\text { displays }\end{array}$ & $\mathrm{Y}$ & $\mathrm{N}$ & 1 & 2 & 3 & 4 & 1 & 2 & 3 & 4 & $\mathrm{Y}$ & $\mathrm{N}$ \\
\hline $\begin{array}{l}\text { 124. Knowledgeable and } \\
\text { experienced staff }\end{array}$ & $\mathrm{Y}$ & $\mathrm{N}$ & 1 & 2 & 3 & 4 & 1 & 2 & 3 & 4 & $\mathrm{Y}$ & $\mathrm{N}$ \\
\hline 125. Customer friendly staff & $\mathrm{Y}$ & $\mathrm{N}$ & 1 & 2 & 3 & 4 & 1 & 2 & 3 & 4 & $\mathrm{Y}$ & $\mathrm{N}$ \\
\hline $\begin{array}{l}\text { 126. Establish a "greeter" at } \\
\text { entrance }\end{array}$ & $\mathrm{Y}$ & $\mathrm{N}$ & 1 & 2 & 3 & 4 & 1 & 2 & 3 & 4 & $\mathrm{Y}$ & $\mathrm{N}$ \\
\hline 127. Add a coffee house & $\mathrm{Y}$ & $\mathrm{N}$ & 1 & 2 & 3 & 4 & 1 & 2 & 3 & 4 & $\mathrm{Y}$ & $\mathrm{N}$ \\
\hline 128. Add a bakery & $\mathrm{Y}$ & $\mathrm{N}$ & 1 & 2 & 3 & 4 & 1 & 2 & 3 & 4 & $\mathrm{Y}$ & $\mathrm{N}$ \\
\hline 129. Add an ice cream shop & Y & $\mathrm{N}$ & 1 & 2 & 3 & 4 & 1 & 2 & 3 & 4 & $\mathrm{Y}$ & $\mathrm{N}$ \\
\hline
\end{tabular}




\begin{tabular}{|c|c|c|c|c|c|c|c|c|c|c|c|c|}
\hline \multirow{3}{*}{ Practice } & \multirow{2}{*}{\multicolumn{2}{|c|}{$\begin{array}{l}\text { Are you } \\
\text { currently } \\
\text { using } \\
\text { this } \\
\text { practice? }\end{array}$}} & \multicolumn{8}{|c|}{ Potential for success in: } & \multirow{2}{*}{\multicolumn{2}{|c|}{$\begin{array}{l}\text { Will you } \\
\text { use this } \\
\text { practice } \\
\text { in the } \\
\text { future? }\end{array}$}} \\
\hline & & & \multicolumn{4}{|c|}{$\begin{array}{l}\text { Getting } \\
\text { customers to } \\
\text { return? }\end{array}$} & \multicolumn{4}{|c|}{$\begin{array}{l}\text { Raising profit } \\
\text { margins? }\end{array}$} & & \\
\hline & $\stackrel{\infty}{\nu}$ & 之 & 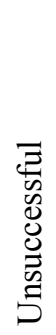 & 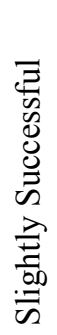 & 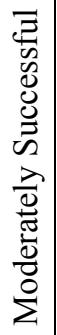 & 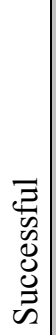 & 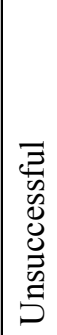 & 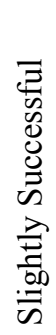 & 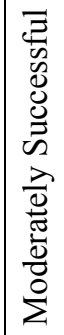 & 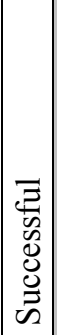 & $\stackrel{\infty}{\infty}$ & ż \\
\hline \multicolumn{13}{|c|}{ Have you made the following adjustments to the business and/or location? (continued) } \\
\hline 130. Raise prices on all items & $\mathrm{Y}$ & $\mathrm{N}$ & 1 & 2 & 3 & 4 & 1 & 2 & 3 & 4 & $\mathrm{Y}$ & $\mathrm{N}$ \\
\hline $\begin{array}{l}\text { 131. Convert greenhouse to } \\
\text { banquet hall during non-peak } \\
\text { months }\end{array}$ & Y & $\mathrm{N}$ & 1 & 2 & 3 & 4 & 1 & 2 & 3 & 4 & $\mathrm{Y}$ & $\mathrm{N}$ \\
\hline $\begin{array}{l}\text { 132. Establish warranty for plant } \\
\text { material }\end{array}$ & $\mathrm{Y}$ & $\mathrm{N}$ & 1 & 2 & 3 & 4 & 1 & 2 & 3 & 4 & $\mathrm{Y}$ & $\mathrm{N}$ \\
\hline 133. Hire a certified horticulturist & $\mathrm{Y}$ & $\mathrm{N}$ & 1 & 2 & 3 & 4 & 1 & 2 & 3 & 4 & $\mathrm{Y}$ & $\mathrm{N}$ \\
\hline 134. Become bonded & $\mathrm{Y}$ & $\mathrm{N}$ & 1 & 2 & 3 & 4 & 1 & 2 & 3 & 4 & $\mathrm{Y}$ & $\mathrm{N}$ \\
\hline 135. & $\mathrm{Y}$ & $\mathrm{N}$ & 1 & 2 & 3 & 4 & 1 & 2 & 3 & 4 & $\mathrm{Y}$ & $\mathrm{N}$ \\
\hline 136. & $\mathrm{Y}$ & $\mathrm{N}$ & 1 & 2 & 3 & 4 & 1 & 2 & 3 & 4 & $\mathrm{Y}$ & $\mathrm{N}$ \\
\hline \multicolumn{13}{|c|}{ Have you focused on customer appreciation by using? } \\
\hline 137. Seasonally dated coupons & $\mathrm{Y}$ & $\mathrm{N}$ & 1 & 2 & 3 & 4 & 1 & 2 & 3 & 4 & $\mathrm{Y}$ & $\mathrm{N}$ \\
\hline 138. Percent-off sale events & $\mathrm{Y}$ & $\mathrm{N}$ & 1 & 2 & 3 & 4 & 1 & 2 & 3 & 4 & $\mathrm{Y}$ & $\mathrm{N}$ \\
\hline $\begin{array}{l}\text { 139. Sell gift certificates at } 20 \% \text { off } \\
\text { during non-peak months }\end{array}$ & $\mathrm{Y}$ & $\mathrm{N}$ & 1 & 2 & 3 & 4 & 1 & 2 & 3 & 4 & $\mathrm{Y}$ & $\mathrm{N}$ \\
\hline 140. Spend $\$ 50$ get $\quad$ free & $\mathrm{Y}$ & $\mathrm{N}$ & 1 & 2 & 3 & 4 & 1 & 2 & 3 & 4 & $\mathrm{Y}$ & $\mathrm{N}$ \\
\hline $\begin{array}{l}\text { 141. Offer extra stamps on loyalty } \\
\text { cards in non-peak months }\end{array}$ & $\mathrm{Y}$ & $\mathrm{N}$ & 1 & 2 & 3 & 4 & 1 & 2 & 3 & 4 & $\mathrm{Y}$ & $\mathrm{N}$ \\
\hline $\begin{array}{l}\text { 142. Offer specials to customers in } \\
\text { costume on Halloween }\end{array}$ & $\mathrm{Y}$ & $\mathrm{N}$ & 1 & 2 & 3 & 4 & 1 & 2 & 3 & 4 & $\mathrm{Y}$ & $\mathrm{N}$ \\
\hline $\begin{array}{l}\text { 143. Holiday specials on } \\
\text { corresponding plants and } \\
\text { accessories i.e. Memorial Day: } \\
\text { red, white and blue }\end{array}$ & $\mathrm{Y}$ & $\mathrm{N}$ & 1 & 2 & 3 & 4 & 1 & 2 & 3 & 4 & $\mathrm{Y}$ & $\mathrm{N}$ \\
\hline 144. & $\mathrm{Y}$ & $\mathrm{N}$ & 1 & 2 & 3 & 4 & 1 & 2 & 3 & 4 & $\mathrm{Y}$ & $\mathrm{N}$ \\
\hline 145. & $\mathrm{Y}$ & $\mathrm{N}$ & 1 & 2 & 3 & 4 & 1 & 2 & 3 & 4 & $\mathrm{Y}$ & $\mathrm{N}$ \\
\hline
\end{tabular}


Instructions: Please check the appropriate response to each of the following questions.

146. Would it be better to only open the garden center in the spring and summer?

a. Yes

b. No

147. How many years has your operation been in business?
a. Less than 5 years
b. 6-10 years
c. 11-15 years
d. 16-20 years
e. 20 or more years

148. What is your age?

a. Less than 20 years

b. 20-29 years

c. 30-39 years

d. $40-49$ years

e. 50-59 years

f. 60-69 years

g. 70-79 years

h. More than 80 years

149. What is your gender?

a. Male

b. Female

150. What is the highest level of education you have completed?

a. High school diploma

b. Two year post secondary degree

c. Associates degree

d. Bachelors degree

e. Masters degree

f. Doctorate degree

151. What are your current dates of operation?

a. Open year round

b. Open spring and summer; Closed fall and winter

c. Other (please specify)

152. Are you a certified horticulturist through the American Society for Horticultural Science?

a. Yes

b. No 
Instructions: Please fill in the blanks for the following questions.

153. What is the approximate square footage of your operation?

a. Square footage of indoor or covered facilities

sq. ft.

b. Square footage of outdoor or uncovered facilities sq. ft.

154. What are your current hours of operation?
a. Monday - Friday open;
b. Saturday close
c. Sunday open; open; close close

155. Would you like to receive a copy of the results of this study? (Please circle)

YES NO

Name:

Address:

City:

State: Zip Code:

Comments:

If you have questions about the survey, please contact me at: nhunt@mix.wvu.edu or call (304) 692-5686

Thank you far taking the time ta camplete this surwey. 
APPENDIX E:

Initial Cover Letter 
May 4, 2007

Dear Garden Center Manager:

The bond between horticulture and humans is longstanding and all-encompassing. Garden Centers have helped strengthen this bond as well as the entire horticulture industry. Traditionally, garden centers boom in the spring but business tends to slow down in the summer. Modern garden centers are trying to revolutionize this tradition by successfully staying open year round. By taking the time to answer this survey, you too can be a part of the revolution! Your contribution is vital to the success of this study and the horticulture industry.

The purpose of this research study is to determine successful practices being utilized by independent retail garden centers to extend the profitable season beyond the spring and early summer months. The results of this study will be used to prepare a thesis to partially fulfill the requirements for a Master of Science Degree in Agricultural and Extension Education. By determining what is currently being practiced to extend the profitable season, other garden centers might be able to improve their own profit margins as well.

Participation in this research study is completely voluntary and all information you provide will be held as confidential as possible. Your response to the survey is critical to the success of the study and will only take a few minutes of your time to complete. A copy of the results of the study will be available upon request. You may skip any question you are not comfortable answering. You will notice a code number at the top left of the return envelope. This code will be used to identify non-respondents for follow-up purposes and will be destroyed before the data are analyzed. Survey results will be reported in a summary format and individual responses will not be identifiable.

Please place the completed questionnaire in the enclosed postage-paid selfaddressed return envelop and drop it in the mail. Please return your completed questionnaire before May 15, 2007. We thank you in advance for your assistance with this research effort. We sincerely appreciate your time and effort.

The instrument and all supporting documents have been reviewed by the West Virginia University Institutional Review Board and are on file.

Sincerely,

Nona Kay Hunt

Graduate Student
Stacy A. Gartin

Professor \& Chair 
APPENDIX F:

Follow-Up Cover Letter 
June 1, 2007

Dear Garden Center Manager:

The bond between horticulture and humans is longstanding and all-encompassing. Garden Centers have helped strengthen this bond as well as the entire horticulture industry. Traditionally, garden centers boom in the spring but business tends to slow down in the summer. Modern garden centers are trying to revolutionize this tradition by successfully staying open year round. By taking the time to answer this survey, you too can be a part of the revolution! Your contribution is vital to the success of this study and the horticulture industry.

The purpose of this research study is to determine successful practices being utilized by independent retail garden centers to extend the profitable season beyond the spring and early summer months. The results of this study will be used to prepare a thesis to partially fulfill the requirements for a Master of Science Degree in Agricultural and Extension Education. By determining what is currently being practiced to extend the profitable season, other garden centers might be able to improve their own profit margins as well.

Participation in this research study is completely voluntary and all information you provide will be held as confidential as possible. Your response to the survey is critical to the success of the study and will only take a few minutes of your time to complete. A copy of the results of the study will be available upon request. You may skip any question you are not comfortable answering. You will notice a code number at the top left of the return envelope. This code will be used to identify non-respondents for follow-up purposes and will be destroyed before the data are analyzed. Survey results will be reported in a summary format and individual responses will not be identifiable.

Please place the completed questionnaire in the enclosed postage-paid selfaddressed return envelop and drop it in the mail. Please return your completed questionnaire before June 13, 2007. We thank you in advance for your assistance with this research effort. We sincerely appreciate your time and effort. If you have already returned your questionnaire, please disregard this mailing.

Sincerely,

Nona Kay Hunt

Graduate Student
Stacy A. Gartin

Professor \& Chair 
APPENDIX G:

Follow-Up Postcard 
You recently received a questionnaire as part of my thesis study to determine the current practices utilized by independent retail garden centers to extend the profitable season. I realize this is a busy time of year but it will only take a few minutes of your time to answer the survey. Your contribution is vital to the success of this study and the horticulture industry. The results of the study could help you enhance the profitability of your business and are available upon request.

Thank you for your time!

Nona Kay Hunt

Agricultural and Extension Education

Davis College of Agriculture, Forestry, and Consumer Sciences

West Virginia University

2048 Agricultural Sciences Building

P.O. Box 6108

Morgantown, WV 26506-6108 
APPENDIX H:

Respondent Comments 
We operate a tree farm and full service back yard nursery. We do landscaping and tree service work. We are trying not to be open all year. The November, December Christmas lot is very successful. We sell cut trees and make a large number of decorations. We plant to quit in the next few years.

I enjoy helping students complete their projects. If you do a survey in the future, I suggest you send it during down times-not the busiest part of the year. I originally received this survey in May-there are more pressing things to do than answer a survey. I wish you success in your studies and future employment.

We are currently in our $5^{\text {th }}$ year of operation and $4^{\text {th }}$ year at this site.

* We are family owned and operated.

* We have a spring open house the weekend before Mother's Day each year that we coincide with our customer appreciation day and offer free lunch to any and all that stop by that day. (over 1000 this year)

* We donate product and our services to local charities.

* We have customers that drive 50 miles and pass several garden centers to shop with us for our product and services.

You had some neat ideas we may want to try! Thanks!

We are small, deal mostly in herbs and trees.

Sorry I am late. Wrong time of year for office work!! Hope this can be included in study.

We are closing are doors in 1 month. We are mainly a perennial retailer. We feel we have done many things well. But it just hasn't worked out. If you're interested this could be a great case study. But out of the scope of this survey. But it has been a great story and my heart breaks at our closing.

Customer count does not increase margins. Don't confuse "sales" with margins. Margins are determined by the difference between purchase price after beginning and ending inventory levels have been factored. Small businesses think "more traffic equals more sales equals more profit". That is not always the case.

We deliver bulk landscape supplies to the retail and commercial gardener/landscaper. 
VITA

Nona Kay Hunt

May 2002

July 2002-July 2003

October 2003

February-April 2006

May 2006

January-May 2007

May 2008
Graduated-Roane County High School

Spencer, WV 25276

State Secretary

West Virginia FFA Association

Earned American FFA Degree

Student Teacher

Hundred High School

Hundred, WV 26575

Bachelor of Science in Agriculture

Agricultural and Environmental Education

West Virginia University

Morgantown, WV 26506

Graduate Teaching Assistant

AGEE 421-Agricultural \& Natural Resources

Communications

West Virginia University

Morgantown, WV 26506

Master of Science

Agricultural and Extension Education

West Virginia University

Morgantown, WV 26506 